\title{
DIFFERENCE EQUATIONS ARISING FROM CLUSTER ALGEBRAS
}

\author{
YUMA MIZUNO
}

\begin{abstract}
We characterize $\mathrm{Y} / \mathrm{T}$-system type difference equations arising from cluster algebras by triples of matrices, which we call T-data, that have a certain symplectic property. We show that all mutation loops are essentially obtained from T-data, which generalizes the general solution for period 1 quivers given by Fordy and Marsh. We also show that any T-datum associated with a periodic Y/T-system has the simultaneous positivity. As an application, we propose a version of Nahm's conjecture from a viewpoint of cluster algebras. We conjecture that given a periodic $\mathrm{T} / \mathrm{Y}$-system of a certain type, we have a family of hypergeometric $q$-series that are also modular functions.
\end{abstract}

\section{Contents}

1. Introduction

2. $\mathrm{Y} / \mathrm{T}$-systems in cluster algebras

2.1. Preliminaries on cluster algebras

2.2. T-systems and Y-systems in cluster algebras

2.3. Relation between Y-systems and T-systems

3. Axiomatic approach to $\mathrm{Y} / \mathrm{T}$ systems

3.1. T-data

3.2. T-data from mutation loops

3.3. Mutation loops from T-data

3.4. Consequences

3.5. Tropical T-system

3.6. Indecomposable T-data 23

4. Examples of T-data 24

4.1. Period 1 quivers 24

4.2. Commuting Cartan matrices 25

4.3. T-systems associated with quantum affinizations 28

5. Periodic Y/T-systems 32

5.1. Finite type T-data 32

5.2. Simultaneous positivity of finite type T-data 33

5.3. Special values of the dilogarithm function 35

5.4. Partition $q$-series $\quad 37$

References

\section{INTRODUCTION}

Cluster algebras were introduced by Fomin and Zelevinsky in the seminal paper [11]. A cluster algebra is a commutative ring equipped with a combinatorial structure called a cluster pattern. A cluster pattern is a graph whose vertices are clusters, which are tuples of cluster variables, and edges are exchange relations. 
Such combinatorial structures have been found in many areas of mathematics, and thus the theory of cluster algebra has many applications.

One of the main applications of the theory of cluster algebras is the study of discrete dynamical systems. In their fourth paper on cluster algebras [14], Fomin and Zelevinsky introduced bipartite belts, which are discrete dynamical systems associated with bipartite symmetrizable generalized Cartan matrices. They proved that the bipartite belt associated with a generalized Cartan matrix $A$ is periodic if and only if $A$ is of finite type, that is, there exists a vector $v>0$ such that $A v>0$. Thus, periodic bipartite belts are classified by the Cartan-Killing classification. This result generalizes and refines the periodicity of Zamolodchikov's Y-systems, which was conjectured by Zamolodchikov [51] in the study of thermodynamic Bethe ansatz, and proved by Fomin and Zelevinsky in [13] prior to their fourth paper [14]. They also proved that there is a bijection between the set of terms appear in a bipartite belt associated with a finite type Cartan matrix $A$ and the set of almost positive roots in the root system associated with $A$. A key fact in the proof of these results is that terms in a bipartite belt are realized as cluster variables in some cluster algebra, and recurrence relations of this bipartite belt are realized as exchange relations in the same cluster algebra.

Bipartite belts are very special cases of discrete dynamical systems called $Y$-systems and T-systems in cluster algebras, in the sense of Nakanishi's paper [41]. These discrete dynamical systems have nice properties inherited from general properties of cluster algebras such as the Laurent phenomenon [11], the Laurent positivity [38, 23], the synchronicity phenomenon [43], and the quantization [2, 9]. It has been discovered that many interesting discrete dynamical systems can be realized as Y-systems or T-systems in cluster algebras, for example:

- periodic discrete dynamical systems that are generalization of Zamolodchikov's Y-systems [14, 19, $27,28,33,44]$

- non-periodic but integrable discrete dynamical systems such as Q-systems [6, 7, 32], pentagram maps [20, 22], the $q$-Painlevé equations [3, 26, 45], and discrete dynamical systems associated with mutation-periodic quivers [15, 16] and bipartite recurrent quivers [17, 18].

Because of these nice properties and interesting examples, it is natural to ask what discrete dynamical systems arise from cluster algebras in general. In this paper, we give an answer to this question.

Main result. Let $r$ be a positive integer, and we denote by $[1, r]$ the set $\{1, \ldots, r\}$. Given a triple of matrices $\left(N_{0}, N_{+}, N_{-}\right)$in $\operatorname{Mat}_{r \times r}(\mathbb{Z}[z])$ whose entries are written as

$$
N_{\varepsilon}=\left(\sum_{p \in \mathbb{Z}_{\geq 0}} n_{a b ; p}^{\varepsilon} z^{p}\right)_{a, b \in[1, r]}
$$

we consider the following relation for each $(a, u) \in[1, r] \times \mathbb{Z}$ among indeterminates in $\left\{T_{a}(u) \mid(a, u) \in\right.$ $[1, r] \times \mathbb{Z}\}:$

$$
\prod_{b=1}^{r} \prod_{p \geq 0} T_{b}(u+p)^{n_{b a ; p}^{0}}=\prod_{b=1}^{r} \prod_{p \geq 0} T_{b}(u+p)^{n_{b a ; p}^{+}}+\prod_{b=1}^{r} \prod_{p \geq 0} T_{b}(u+p)^{n_{b a ; p}^{-}}
$$

We impose the following conditions on $\left(N_{0}, N_{+}, N_{-}\right)$:

$$
\begin{aligned}
& \text { (N1) } n_{a b ; p}^{0}=\delta_{a b} \delta_{p 0}+\delta_{a \sigma(b)} \delta_{p p_{a}} \text { for some } \sigma \in \mathfrak{S}_{r} \text { and } p_{a} \in \mathbb{Z}_{>0}, \\
& \text { (N2) } n_{a b ; p}^{+} \geq 0 \text { and } n_{a b ; p}^{-} \geq 0 \text { for any } a, b, p, \\
& \text { (N3) } n_{a b ; p}^{+}=0 \text { and } n_{a b ; p}^{-}=0 \text { unless } 0<p<p_{a}, \\
& \text { (N4) } n_{a b ; p}^{+} n_{a b ; p}^{-}=0 \text { for any } a, b, p,
\end{aligned}
$$

where $\mathfrak{S}_{r}$ is the symmetric group on $[1, r]$ and $\delta$ is the Kronecker delta. The condition (N1) says that the left-hand side in (1.1) is equal to $T_{a}(u) T_{\sigma(a)}\left(u+p_{\sigma(a)}\right)$. The condition (N2) says that the right-hand side in (1.1) is a sum of two monomials. The condition (N3) together with (N1) implies that any $T_{a}(u)$ can be 
written as a rational function in the initial variables $\left(T_{a}(p)\right)_{(a, p) \in R_{\text {in }}}$, where

$$
R_{\text {in }}=\left\{(a, p) \in[1, r] \times \mathbb{Z} \mid 0 \leq p<p_{a}\right\} .
$$

The condition (N4) says that the two monomials in the right-hand side in (1.1) do not have common divisors.

Definition 1.1. We say that a triple of matrices $\alpha=\left(A_{+}, A_{-}, D\right)$ is a T-datum of size $r$ if $A_{ \pm}$can be written as $A_{ \pm}=N_{0}-N_{ \pm}$by a triple of matrices $\left(N_{0}, N_{+}, N_{-}\right)$in $\operatorname{Mat}_{r \times r}(\mathbb{Z}[z])$ satisfying (N1)-(N4), and $D$ is a positive integer diagonal matrix satisfying the following conditions:

- $N_{0} D=D N_{0}$,

- $D^{-1} N_{ \pm} D \in \operatorname{Mat}_{r \times r}(\mathbb{Z}[z])$,

- $A_{+} D A_{-}^{\dagger}=A_{-} D A_{+}^{\dagger}$,

where $A_{ \pm}^{\dagger}:=\left(\left.A_{ \pm}\right|_{z=z^{-1}}\right)^{\top}$.

Definition 1.2. Let $\alpha$ be a T-datum. Let $\mathcal{T}(\alpha)$ be the commutative ring generated by the indeterminates $\left(T_{a}(u)^{ \pm 1}\right)_{(a, u) \in[1, r] \times \mathbb{Z}}$ subject to the relations $(1.1)$ and $T_{a}(u) T_{a}(u)^{-1}=1$ for any $(a, u) \in[1, r] \times \mathbb{Z}$. We

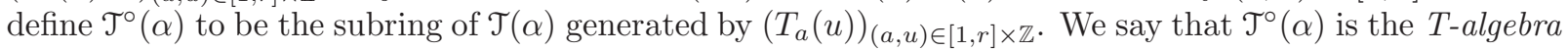
associated with $\alpha$. We also say that the family of relations (1.1) is the T-system associated with $\alpha$.

Let $I$ be a finite index set. For a pair $(B, x)$ of an $I \times I$ skew-symmetrizable integer matrix $B$ and an $I$-tuple $x=\left(x_{i}\right)_{i \in I}$ of algebraically independent commuting variables, the cluster algebra associated with the initial seed $(B, x)$ is defined $[11,14]$, which is denoted by $\mathcal{A}(B, x)$. In Section 3.4 , we prove the following:

Theorem 1.3. Let $\alpha$ be a T-datum of size $r$. Let $x=\left(x_{a, p}\right)_{(a, p) \in R_{\mathrm{in}}}$ be an $R_{\mathrm{in}}$-tuple of algebraically independent commuting variables. Then there exists a unique $R_{\mathrm{in}} \times R_{\mathrm{in}}$ skew-symmetrizable integer matrix $B$ such that

(1) there exists a unique injective ring homomorphism $\iota: \mathcal{T}^{\circ}(\alpha) \hookrightarrow \mathcal{A}(B, x)$ such that $\iota\left(T_{a}(p)\right)=x_{a, p}$ for any $(a, p) \in R_{\text {in }}$,

(2) $\iota\left(T_{a}(u)\right)$ is a cluster variable in $\mathcal{A}(B, x)$ for any $(a, u) \in[1, r] \times \mathbb{Z}$,

(3) the image of the relation (1.1) by $\iota$ is an exchange relation in $\mathcal{A}(B, x)$ for any $(a, u) \in[1, r] \times \mathbb{Z}$.

Conversely, we also prove that T-systems in cluster algebras (in the sense in [41]) yield T-data (Section 2.2 and 3.2). Therefore, our definition of T-data completely characterize when a system of difference equations of the form (1.1) is realized as a family of exchange relations in a cluster algebra. In the following, we give remarks and applications of Theorem 1.3.

Sequences of mutations that preserve exchange matrices. The matrix $B$ in Theorem 1.3 is called the initial exchange matrix in the cluster algebra $\mathcal{A}(B, x)$. In the proof of Theorem 1.3, we give the explicit formula (3.9) expressing $B$ using a matrix coefficients in a T-datum. We also construct a sequence of mutations, which are fundamental operations in the theory of cluster algebras, that preserves the exchange matrix $B$ up to relabeling of indices. Such a sequence of mutations is called a mutation loop.

Mutation loops themselves are of interest from a geometric viewpoint: they are representatives of elements in cluster modular groups [9], which are cluster algebraic counterparts of mapping class groups of surfaces. We show that essentially all mutation loops are obtained by the formula (3.9) (Theorem 3.18). The formula (3.9) gives a effective way to find mutation loops since finding T-data is usually easier than finding mutation loops. We give many examples of T-data in Section 4, which recover or generalize mutation loops in the literature. In Section 4.1, we classify T-data of size 1 (Theorem 4.1), which turns out to recover the classification of period 1 quivers by Fordy and Marsh [16]. In Section 4.2, we define T-data associated with pairs of commuting Cartan matrices. They are generalization of bipartite belts by Fomin and Zelevinsky [14]. In particular, our definition also works for non-bipartite cases such as the "tadpole type". In Section 4.3, we define T-data associated with level restricted T-systems for quantum affinizations [35]. These T-systems are restricted version of T-systems for quantum affinizations discovered by 
Hernandez [25], where "T-systems for quantum affinizations" mean algebraic relations among $q$-characters of Kirillov-Reshetikhin modules over quantum affinizations. Although mutation loops corresponding to these T-data are already constructed in [27, 28, 35, 42], our method gives a simple systematic way to produce these mutation loops.

T-systems with coefficients and Y-systems. Theorem 1.3 can be extended to T-systems with coefficients. In fact, we show Theorem 1.3 in this generality (Theorem 3.19). Coefficients of T-systems are governed by Y-systems, which are generalization of Zamolodchikov's Y-systems [51]. In terms of T-data, the coefficients of the T-system associated with a T-datum $\alpha$ is described by the Langlands dual T-datum $\alpha^{\vee}=\left(A_{+}^{\vee}, A_{-}^{\vee}, D^{\vee}\right)$. If we write the entries of the matrices in $\alpha^{\vee}$ as

$$
N_{\varepsilon}^{\vee}=\left(\sum_{p \in \mathbb{Z}_{\geq 0}} \check{n}_{a b ; p}^{\varepsilon} z^{p}\right)_{a, b \in[1, r]}
$$

the coefficients of the T-system associated with $\alpha$ is governed by the following system of relations:

$$
\prod_{b=1}^{r} \prod_{p \geq 0} Y_{b}(u-p)^{\check{n}_{a b ; p}^{0}}=\frac{\prod_{b=1}^{r} \prod_{p \geq 0}\left(1 \oplus Y_{b}(u-p)\right)^{\check{n}_{a b ; p}^{-}}}{\prod_{b=1}^{r} \prod_{p \geq 0}\left(1 \oplus Y_{b}(u-p)^{-1}\right)^{\check{n}_{a b ; p}^{+}}}
$$

where $\oplus$ is the "auxiliary addition" in the underlying semifield to which the coefficients belong. We call this family of relations the Y-system associated with $\alpha$.

Periodic T-systems and Y-systems. We say that a T-datum is of finite type if the set $\left\{T_{a}(u) \in \mathcal{T}^{\circ}(\alpha) \mid\right.$ $(a, u) \in[1, r] \times \mathbb{Z}\}$ is a finite set. This is equivalent to saying that the T-system associated with $\alpha$ is periodic. By the synchronicity phenomenon of cluster algebras [43], this is also equivalent to the periodicity of the $Y$-system associated with $\alpha$ in universal semifields.

Many examples of finite type T-data have been found in the literature, which are associated with the following data:

- finite type Cartan matrices [51, 13, 14],

- tensor products of pairs of finite type Cartan matrices [46, 33],

- untwisted quantum affine algebras [34, 27, 28],

- the sine-Gordon Y-systems and the reduced sine-Gordon Y-systems [48, 44], which are associated with continued fractions,

- admissible $A D E$ bigraphs [19].

In many cases in this list, the periodicities of Y-systems in universal semifields were conjectured in the 1990 s in physics $[51,46,34,48]$, and proved in the 21 st century by using the theory of cluster algebras [13, $14,33,27,28,44,19]$.

Since there are many interesting examples of finite type T-data as in this list, the classification of finite type T-data is a interesting problem. Except for special cases [14, 19], however, the classification of finite type T-data is still not well understood. In this paper, we prove that any finite type T-datum satisfies the following simultaneous positivity:

Theorem 1.4 (Theorem 5.5). Let $\alpha=\left(A_{+}, A_{-}, D\right)$ be a T-datum. If $\alpha$ is of finite type, then there exists a vector $v>0$ such that $\AA_{+}^{\top} v>0$ and $\AA_{-}^{\top} v>0$, where $\AA_{ \pm}=\left.A_{ \pm}\right|_{z=1}$.

Theorem 1.4 gives a effective method to determine that a given T-datum is not of finite type (see Example 5.6). This theorem is also used in the next topic: relationship between cluster algebras and Nahm's conjecture.

Nahm's conjecture. In [40], Nahm gave a connection between rational conformal field theories and torsion elements in Bloch groups. In particular, Nahm's conjecture states that the modularity of certain hypergeometric $q$-series is related to torsion elements in Bloch groups (see [50, Chapter II, Section 3]). We give a version of Nahm's conjecture from a viewpoint of cluster algebras. 
Let $\alpha=\left(A_{+}, A_{-}, D\right)$ be a Cartan-like T-datum (see Definition 5.7) of finite type. By Theorem 1.4, we can show that the system of equations

$$
f_{a}=\prod_{b=1}^{r}\left(1-f_{b}\right)^{\check{\kappa}_{a b}} \quad(a \in[1, r])
$$

has a unique real solution such that $0<f_{a}<1$ for any $a \in[1, r]$, where we define $K^{\vee}=\left(\check{\kappa}_{a b}\right) \in \operatorname{Mat}_{r \times r}(\mathbb{Q})$ by $K^{\vee}=\left(\AA_{+}^{\vee}\right)^{-1} \AA_{-}^{\vee}$. For this solution, the value

$$
c_{\alpha}:=\frac{6}{\pi^{2}} \sum_{a=1}^{r} d_{a} L\left(f_{a}\right)
$$

turns out to be a rational number, where $L(x)$ is the Rogers dilogarithm function. This fact follows from dilogarithm identities in cluster algebras that are proved by Nakanishi [41]. Moreover, for any solution $\left(f_{1}, \ldots, f_{r}\right) \in \overline{\mathbb{Q}}^{r}$ of $(1.2)$, we can define a torsion element in the Bloch group $\mathcal{B}(F)$, where $F$ is a number field containing the solution.

Motivated by Nahm's conjecture, we introduce a family of hypergeometric $q$-series $\left(\mathcal{Z}_{\alpha, \sigma}(q)\right)_{\sigma \in S_{\alpha}}$ for any Cartan-like T-datum $\alpha$ of finite type, where $S_{\alpha}$ is a finite abelian group associated with $\alpha$. We call these $q$-series the partition q-series of $\alpha$. In fact, these are generalization of partition $q$-series of mutation loops introduced by Kato and Terashima [31]. We conjecture that partition $q$-series are modular functions:

Conjecture 1.5 (Conjecture 5.14). Let $\alpha$ be a Cartan-like T-datum of finite type. Then $q^{-c_{\alpha} / 24} Z_{\alpha, \sigma}(q)$ is a modular function for any $\sigma \in S_{\alpha}$, where $c_{\alpha}$ is the rational number defined by (1.3).

We prove this conjecture for $r=1$ using Rogers-Ramanujan type identities (Theorem 5.16). We also give the following examples (Example 5.17-5.20) supporting the conjecture for $r \geq 2$ : Zagier's lists of $2 \times 2$ and $3 \times 3$ matrices concerning the Nahm's conjecture [50], a $q$-series in the Andrew-Gordon identity [1], fermionic formulas for quantum affine algebras [24], and $q$-series appear in the theory of nilpotent double affine Hecke algebras [5].

Acknowledgment. The author thanks Yuji Terashima and Shunsuke Kano for fruitful discussions. This work is supported by JSPS KAKENHI Grant Number JP18J22576.

\section{Y/T-Systems In CLUSTER ALGEBRAS}

2.1. Preliminaries on cluster algebras. We review cluster algebras following [14]. Let $I$ be a finite index set. An $I \times I$ integer matrix $B=\left(B_{i j}\right)_{i, j \in I}$ is called skew-symmetrizable if there exist a tuple of positive integers $d=\left(d_{i}\right)_{i \in I}$ such that $B_{i j} d_{j}=-B_{j i} d_{i}$. Such a tuple is called a (right) symmetrizer of $B$. For any $I \times I$ matrix $B=\left(B_{i j}\right)_{i, j \in I}$ and bijection $\nu: I \rightarrow I^{\prime}$ between finite index sets, we define an $I^{\prime} \times I^{\prime}$ matrix $\nu(B)=\left(B_{i^{\prime} j^{\prime}}^{\prime}\right)_{i^{\prime}, j^{\prime} \in I^{\prime}}$ by $B_{\nu(i) \nu(j)}^{\prime}=B_{i j}$.

Definition 2.1. Let $B=\left(B_{i j}\right)_{i, j \in I}$ be a skew-symmetrizable integer matrix, and let $k \in I$. The matrix mutation $\mu_{k}: B \mapsto B^{\prime}$ is a transformation that transforms $B$ into a new skew-symmetrizable integer matrix $B^{\prime}=\left(B_{i j}^{\prime}\right)_{i, j \in I}$ defined as follows:

$$
B_{i j}^{\prime}= \begin{cases}-B_{i j} & \text { if } i=k \text { or } j=k, \\ B_{i j}+\left[B_{i k}\right]_{+}\left[B_{k j}\right]_{+}-\left[-B_{i k}\right]_{+}\left[-B_{k j}\right]_{+} & \text {otherwise },\end{cases}
$$

where $[x]_{+}:=\max (0, x)$.

If $d$ is a symmetrizer of $B$, then it is also a symmetrizer of $\mu_{k}(B)$. In particular, if $B$ is a skew-symmetric integer matrix, then $\mu_{k}(B)$ is also a skew-symmetric integer matrix. In this case, it is convenient to describe matrix mutations in terms of quivers. A quiver is a finite oriented graph without loops and 2-cycles. For any skew-symmetric integer matrix $B$, we define a quiver $Q(B)$ as follows: the vertex set is $I$, and there are $\left[B_{i j}\right]_{+}$arrows from $i$ to $j$. Conversely, we can recover a skew-symmetric integer matrix $B(Q)$ from a 
quiver $Q$ by $B(Q)_{i j}=Q_{i j}-Q_{j i}$, where $Q_{i j}$ is the number of arrows from $i$ to $j$. This gives a bijection between the set of $I \times I$ skew-symmetric integer matrices and the set of quivers whose vertex set is $I$.

Definition 2.2. Let $Q$ be a quiver, and let $k$ be a vertex of $Q$. The quiver mutation $\mu_{k}$ is a transformation that transforms $Q$ into a quiver $\mu_{k}(Q)$ defined by the following three steps:

(1) For each length two path $i \rightarrow k \rightarrow j$, add a new arrow $i \rightarrow j$.

(2) Reverse all arrows incident to the vertex $k$.

(3) Remove all 2-cycles.

Matrix mutations and quiver mutations are compatible. The transformation
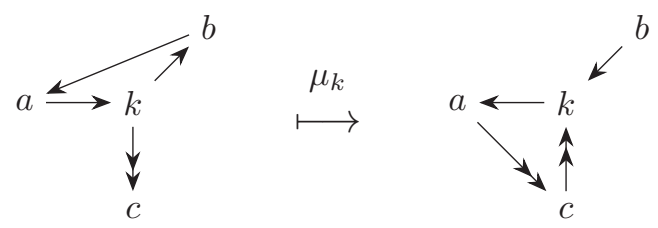

is an example of a quiver mutation.

A set $\mathbb{P}$ is called a semifield if it is an abelian multiplicative group endowed with an binary operation $\oplus$ which is commutative, associative, and distributive with respect to the multiplication. We denote by $\mathbb{Z P}$ the group ring of $\mathbb{P}$ over $\mathbb{Z}$. This ring is an integral domain since the abelian multiplicative group of $\mathbb{P}$ is torsion-free. Throughout this paper, a $\mathbb{Z} \mathbb{P}$-algebra means a commutative associative $\mathbb{Z} \mathbb{P}$-algebra with an identity element, and we assume that a $\mathbb{Z} \mathbb{P}$-algebra homomorphism sends the identity element to the identity element. We denote by $\mathbb{Q P}$ the field of fractions of $\mathbb{Z P}$. We fix a field $\mathcal{F}$ that is isomorphic to the field of rational functions over $\mathbb{Q P}$ in $|I|$ variables.

Example 2.3. Let $J$ be a finite index set.

(1) Let $\operatorname{Trop}\left(u_{j}\right)_{j \in J}$ be the abelian multiplicative group generated by the indeterminates $\left(u_{j}\right)_{j \in J}$. We define a binary operation $\oplus$ on $\operatorname{Trop}\left(u_{j}\right)_{j \in J}$ by

$$
\prod_{j \in J} u_{j}^{a_{j}} \oplus \prod_{j \in J} u_{j}^{b_{j}}=\prod_{j \in J} u_{j}^{\min \left(a_{j}, b_{j}\right)} .
$$

This binary operation makes $\operatorname{Trop}\left(u_{j}\right)_{j \in J}$ a semifield, which is called a tropical semifield. If $J$ is the empty set, $\operatorname{Trop}\left(u_{j}\right)_{j \in J}=\{1\}$ is called the trivial semifield.

(2) Let $\mathbb{Q}_{\text {sf }}\left(u_{j}\right)_{j \in J}$ be the subset of $\mathbb{Q}\left(u_{j}\right)_{j \in J}$ consisting of all rational functions that can be written as subtraction-free expressions in $\left(u_{i}\right)_{i \in J}$. The set $\mathbb{Q}_{s f}\left(u_{j}\right)_{j \in J}$ is a semifield with respect to the usual multiplication and addition, which is called a universal semifield.

Definition 2.4. An (I-labeled) $Y$-seed in $\mathbb{P}$ is a pair $(B, y)$, where

- $B=\left(B_{i j}\right)_{i, j \in I}$ is an $I \times I$ skew-symmetrizable integer matrix,

- $y=\left(y_{i}\right)_{i \in I}$ is an $I$-tuple in elements of $\mathbb{P}$.

Definition 2.5. An (I-labeled) seed in $\mathcal{F}$ is a pair $(B, y, x)$, where

- $(B, y)$ is an $I$-labeled Y-seed in $\mathbb{P}$,

- $x=\left(x_{i}\right)_{i \in I}$ is an $I$-tuple of elements in $\mathcal{F}$ forming a free generating set, that is, $\left(x_{i}\right)_{i \in I}$ is algebraically independent over $\mathbb{Q P}$, and $\mathcal{F}=\mathbb{Q P}\left(x_{i}\right)_{i \in I}$.

For a seed $(B, y, x)$, we refer to $B$ as the exchange matrix, $y$ as the coefficient tuple, $x$ as the cluster, $y_{i}$ 's as the coefficients, and $x_{i}$ 's as the cluster variables.

Definition 2.6. Let $(B, y, x)$ be an $I$-labeled seed in $\mathcal{F}$, and let $k \in I$. The seed mutation $\mu_{k}:(B, y, x) \mapsto$ $\left(B^{\prime}, y^{\prime}, x^{\prime}\right)$ is a transformation that transforms $(B, x, y)$ into a new seed $\left(B^{\prime}, y^{\prime}, x^{\prime}\right)$ defined as follows:

- $B^{\prime}=\left(B_{i j}^{\prime}\right)_{i, j \in I}$ is given by $(2.1)$, 
- $y^{\prime}=\left(y_{i}^{\prime}\right)_{i \in I}$ is given by

$$
y_{i}^{\prime}= \begin{cases}y_{k}^{-1} & \text { if } i=k, \\ y_{i}\left(1 \oplus y_{k}\right)^{-B_{k i}} & \text { if } i \neq k \text { and } B_{k i} \leq 0, \\ y_{i}\left(1 \oplus y_{k}^{-1}\right)^{-B_{k i}} & \text { if } i \neq k \text { and } B_{k i} \geq 0,\end{cases}
$$

- $x^{\prime}=\left(x_{i}^{\prime}\right)_{i \in I}$ is given by $x_{i}^{\prime}=x_{i}$ if $i \neq k$, and

$$
x_{k}^{\prime}=x_{k}^{-1}\left(\frac{y_{k}}{1 \oplus y_{k}} \prod_{j \in I} x_{j}^{\left[B_{j k}\right]_{+}}+\frac{1}{1 \oplus y_{k}} \prod_{j \in I} x_{j}^{\left[-B_{j k}\right]_{+}}\right) .
$$

We also say that the transformation $\mu_{k}:(B, y) \mapsto\left(B^{\prime}, y^{\prime}\right)$ is the $Y$-seed mutation.

The relation (2.3) is called the exchange relation. Seed mutations are involutions, that is, $\mu_{k}\left(\mu_{k}(B, y, x)\right)=$ $(B, y, x)$.

The $I$-regular tree $\mathbb{T}_{I}$ is the tree such that all vertices have degree $|I|$ and the edges that are incident to each vertex are labeled by the elements in $I$. A cluster pattern is an assignment of an $I$-labeled seed to every vertex in $\mathbb{T}_{I}$, such that the two seeds assigned to the endpoints of any edge labeled by $k \in I$ are obtained from each other by the seed mutation $\mu_{k}$.

Definition 2.7. The cluster algebra $\mathcal{A}$ associated with a given cluster pattern is the $\mathbb{Z} \mathbb{P}$-subalgebra of $\mathcal{F}$ generated by all cluster variables in the pattern. We denote $\mathcal{A}=\mathcal{A}(B, y, x)$, where $(B, y, x)$ is any seed in the underlying cluster pattern. We often denote $\mathcal{A}(B, y, x)$ by $\mathcal{A}(B, x)$ when $\mathbb{P}$ is the trivial semifield.

2.2. T-systems and $\mathbf{Y}$-systems in cluster algebras. In this section, we review T-systems and $\mathrm{Y}$ systems in cluster algebras following [41]. Simply put, T-systems and Y-systems are algebraic relations that $x_{k}$ 's and $y_{k}$ 's, respectively, at mutation indices satisfy.

Let $B=\left(B_{i j}\right)_{i, j \in I}$ be a skew-symmetrizable integer matrix. Let $r$ be a positive integer. For any sequence of indices $\mathbf{i}=\left(i_{1}, \ldots, i_{r}\right) \in I^{r}$, we denote the composition of mutations $\mu_{i_{r}} \circ \cdots \circ \mu_{i_{1}}$ by $\mu_{\mathbf{i}}$. If $B_{i_{a} i_{b}}=0$ for any $a, b \in[1, r]$, it is easy to see that $\mu_{\mathbf{i}}(B)=\mu_{\rho(\mathbf{i})}(B)$ for any permutation $\rho \in \mathfrak{S}_{r}$, where $\mathfrak{S}_{r}$ is the group of bijections on $[1, r]$ and $\rho(\mathbf{i}):=\left(i_{\rho^{-1}(1)}, \ldots, i_{\rho^{-1}(r)}\right)$. We say that a transformation $B \mapsto \mu_{\mathbf{i}}(B)$ is a simultaneous mutation if $B_{i_{a} i_{b}}=0$ for any $a, b \in[1, r]$, and $a \neq b$ implies $i_{a} \neq i_{b}$ for any $a, b \in[1, r]$.

Let $\mathbf{i}=\left(i_{1}, \ldots, i_{r}\right) \in I^{r}$ be a sequence of indices. Consider a partition of $\mathbf{i}$ :

$$
\begin{aligned}
\mathbf{i} & =\mathbf{i}(0)|\mathbf{i}(1)| \cdots \mid \mathbf{i}(t-1), \\
\mathbf{i}(u) & =\left(i(u)_{1}, \ldots, i(u)_{r_{u}}\right), \quad \sum_{u=0}^{t-1} r_{u}=r,
\end{aligned}
$$

where we allow $\mathbf{i}(u)$ to be the empty sequence. Formally, a partition of $\mathbf{i}$ is an order-preserving map $[1, r] \rightarrow\{0, \ldots, t-1\}$ where $t$ is a positive integer. A partition (2.4) is called a partition into simultaneous mutations if all $B(u) \mapsto B(u+1)$ in the following mutation sequence are simultaneous mutations:

$$
B=: B(0) \stackrel{\mu_{\mathrm{i}(0)}}{\longmapsto} B(1) \stackrel{\mu_{\mathrm{i}(1)}}{\longmapsto} \cdots \stackrel{\mu_{\mathrm{i}(t-1)}}{\longmapsto} B(t) .
$$

Definition 2.8. We say that a quadruple $\gamma=(B, d, \mathbf{i}, \nu)$ is a mutation loop if

- $B=\left(B_{i j}\right)_{i, j \in I}$ is a skew-symmetrizable integer matrix,

- $d=\left(d_{i}\right)_{i \in I}$ is a right symmetrizer of $B$,

- $\mathbf{i}=\left(i_{1}, \ldots, i_{r}\right)$ is a sequence of elements in $I$ equipped with a partition into simultaneous mutations $\mathbf{i}=\mathbf{i}(0)|\mathbf{i}(1)| \cdots \mid \mathbf{i}(t-1)$,

- $\nu: I \rightarrow I$ is a bijection such that $\mu_{\mathbf{i}}(B)=\nu(B)$ and $d=\nu(d)$.

The integer $r$ is called the length of $\gamma$. Many examples of mutation loops are given in [41, Section 3].

The partition (2.4) decomposes [1,r] into $t$ parts. We define the subgroup $\mathfrak{S}_{r_{0}, \ldots, r_{t-1}} \subseteq \mathfrak{S}_{r}$, which is isomorphic to $\mathfrak{S}_{r_{0}} \times \cdots \times \mathfrak{S}_{r_{t-1}}$, consisting of permutations that fix the each part as a set. 
Definition 2.9. We say that two mutation loops $\gamma=(B, d, \mathbf{i}, \nu)$ and $\gamma^{\prime}=\left(B^{\prime}, d^{\prime}, \mathbf{i}^{\prime}, \nu^{\prime}\right)$, where $\mathbf{i}=\mathbf{i}(0) \mid$ $\cdots \mid \mathbf{i}(t-1)$ and $\mathbf{i}^{\prime}=\mathbf{i}^{\prime}(0)|\cdots| \mathbf{i}^{\prime}\left(t^{\prime}-1\right)$, are equivalent if there exists a bijection $f: I \rightarrow I^{\prime}$ between the index sets of $B$ and $B^{\prime}$, and a permutation $\rho \in \mathfrak{S}_{r_{0}, \ldots, r_{t-1}}$ such that

- $B^{\prime}=f(B)$,

- $d^{\prime}=f(d)$,

- $t=t^{\prime}$ and $\mathbf{i}^{\prime}(u)=f(\rho(\mathbf{i}(u)))$ for each $u=0, \ldots, t-1$,

- $\nu^{\prime}=f \circ \nu \circ f^{-1}$.

For any mutation loop $\gamma=(B, d, \mathbf{i}, \nu)$, we have the following infinite length mutation sequence that extends (2.5):

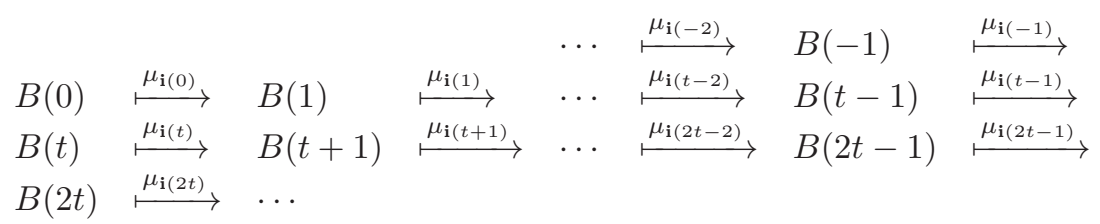

where $B(n t+k)=\nu^{n}(B(k))$ and $\mathbf{i}(n t+k)=\nu^{n}(\mathbf{i}(t+k))$ for any $n \in \mathbb{Z}$ and $0 \leq k \leq t-1$. Let $P_{\gamma}$ be the set defined by

$$
P_{\gamma}=\{(i, u) \in I \times \mathbb{Z} \mid i \in \mathbf{i}(u)\} .
$$

Elements in $P_{\gamma}$ are called mutation points of $\gamma$. We also define an integer $\lambda(i, u)$ for any $(i, u) \in I \times \mathbb{Z}$ by

$$
\lambda(i, u)=\min \left\{v \in \mathbb{Z}_{\geq 0} \mid(i, u+v) \in P_{\gamma}\right\}
$$

if there exists $v \in \mathbb{Z}_{>0}$ such that $(i, u+v) \in P_{\gamma}$. Otherwise, we set $\lambda(i, u)=\infty$. The number $\lambda(i, u)$ is called the latency of $(i, u)$. For any $(i, u) \in I \times \mathbb{Z}$ with $\lambda(i, u)<\infty$, we define an element $s(i, u) \in P_{\gamma}$ by

$$
s(i, u)= \begin{cases}(i, u+\lambda(i, u)) & \text { if }(i, u) \notin P_{\gamma}, \\ (i, u+1+\lambda(i, u+1)) & \text { if }(i, u) \in P_{\gamma} .\end{cases}
$$

The element $s(i, u)$ is called the next mutation point of $(i, u)$.

A mutation loop is called complete if all latencies are finite, that is, $\lambda(i, u)<\infty$ for any $(i, u) \in I \times \mathbb{Z}$, or equivalently, for any $(i, 0) \in I \times\{0\}$. In the rest of this paper, we usually assume that mutation loops are complete.

In order to describe the T-system and the Y-system so that the relationship between them is apparent, we need another parameterization of the mutation points. For any elements $(i, u),(j, v) \in P_{\gamma}$, we write $(i, u) \sim(j, v)$ if there exists $g \in \mathbb{Z}$ such that $j=\nu^{g}(i)$ and $v=u+g t$. Let $\pi: P_{\gamma} \rightarrow[1, r]$ be the surjective map defined by $\pi(i, u)=a$ where $a$ is the unique element in $[1, r]$ such that $(i, u) \sim\left(i_{a}, v\right)$ and $0 \leq v \leq t-1$. We define a set $R_{\gamma}$ by

$$
R_{\gamma}=\left\{(\pi(i, u), u) \mid(i, u) \in P_{\gamma}\right\} .
$$

Lemma 2.10. The map $P_{\gamma} \rightarrow R_{\gamma}$ defined by $(i, u) \mapsto(\pi(i, u), u)$ is a bijection.

Proof. The surjectivity is apparent since $\pi$ is surjective. We assume that $(i, u),(j, u) \in P_{\gamma}$ satisfy $\pi(i, u)=$ $\pi(j, u)$. Then we obtain $(i, u) \sim(j, u)$, and this implies that $i=j$ by the definition of the equivalence relation.

Let $\sigma \in \mathfrak{S}_{r}$ be the bijection defined by

$$
\sigma(a)=\pi(s(i, u)),
$$

where $(i, u) \in \pi^{-1}(a)$ is any mutation point that maps to $a$ by $\pi$. The definition of $\sigma$ does not depend on the choice of $(i, u)$. For any $a \in[1, r]$, we denote by $\lambda_{a}$ the positive integer $1+\lambda(i, u+1)$ where $(i, u) \in \pi^{-1}(a)$. In other words, $\lambda_{a}$ is the positive integer satisfying $s(i, u)=\left(i, u+\lambda_{a}\right)$. The definition of $\lambda_{a}$ also does not depend on the choice of $(i, u)$. 


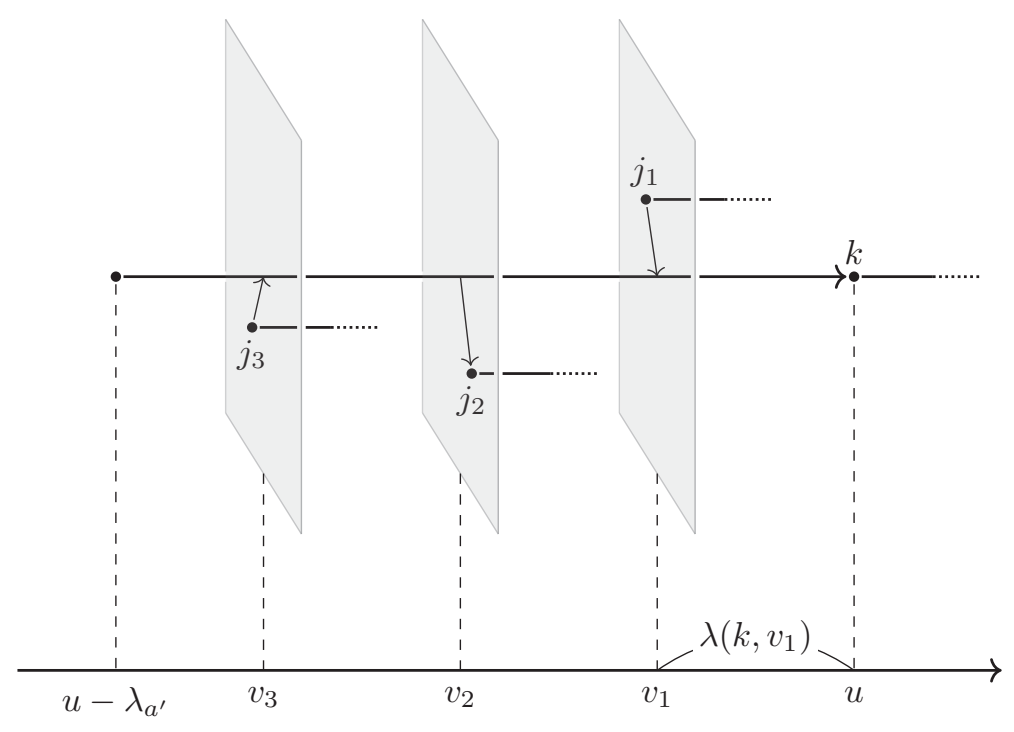

Figure 1. A schematic description of a Y-system. A black point represents a mutation point. An arrow in a plane from (resp. to) a mutation point $(j, v)$ to (resp. from) the right arrow that ends at $(k, u)$ indicates that $\left[B_{j k}(v)\right]_{+} \neq 0\left(\operatorname{resp} .\left[-B_{j k}(v)\right]_{+} \neq 0\right)$.

Let us describe a Y-system associated with a mutation loop $\gamma$. For any Y-seed $(B, y)$, we have the following infinite length sequence of Y-seeds:

$$
\begin{array}{lllll} 
& & \cdots & (B(-1), y(-1)) & \stackrel{\mu_{\mathrm{i}(-1)}}{\longmapsto} \\
(B(0), y(0)) & \stackrel{\mu_{\mathrm{i}(0)}}{\longmapsto} & \cdots & (B(t-1), y(t-1)) & \stackrel{\mu_{\mathrm{i}(t-1)}}{\longmapsto} \\
(B(t), y(t)) & \stackrel{\mu_{\mathrm{i}(t)}}{\longmapsto} & \cdots & &
\end{array}
$$

where $(B(0), y(0))=(B, y)$ and we define negative ones using the involution property of mutations. We define an element $Y_{a}(u) \in \mathbb{P}$ for any $(a, u) \in R_{\gamma}$ by

$$
Y_{a}(u)=y_{i}(u),
$$

where $i \in I$ is a unique index such that $(i, u) \in P_{\gamma}$ and $a=\pi(i, u)$.

Let $N_{0}^{\vee}=\left(\sum_{p \in \mathbb{Z}} \check{n}_{a b ; p^{0}}^{0} z^{p}\right)_{a, b \in[1, r]} \in \operatorname{Mat}_{r \times r}(\mathbb{Z}[z])$ be the $r \times r$ matrix whose entries are integer coefficients polynomials in the variable $z$ defined by

$$
\sum_{p \in \mathbb{Z}} \check{n}_{a b ; p}^{0} z^{p}=\delta_{a b}+\delta_{a^{\prime} b} z^{\lambda_{a^{\prime}}}
$$

where $a^{\prime}=\sigma^{-1}(a)$. We also define two matrices $N_{+}^{\vee}=\left(\sum_{p \in \mathbb{Z}} \check{n}_{a b ; p}^{+} z^{p}\right)_{a, b \in[1, r]}$ and $N_{-}^{\vee}=\left(\sum_{p \in \mathbb{Z}} \check{n}_{a b ; p}^{-} z^{p}\right)_{a, b \in[1, r]}$ in $\operatorname{Mat}_{r \times r}(\mathbb{Z}[z])$ by

$$
\sum_{p \in \mathbb{Z}} \check{n}_{a b ; p^{\prime}}^{ \pm} z^{p}=\sum_{\substack{(j, v) \in P_{\gamma} \\ s(k, v)=(k, u), \pi(j, v)=b}}\left[ \pm B_{j k}(v)\right]_{+} z^{\lambda(k, v)},
$$

where $(k, u) \in \pi^{-1}(a)$. The definition of $N_{ \pm}^{\vee}$ does not depend on the choices of $(k, u)$. 


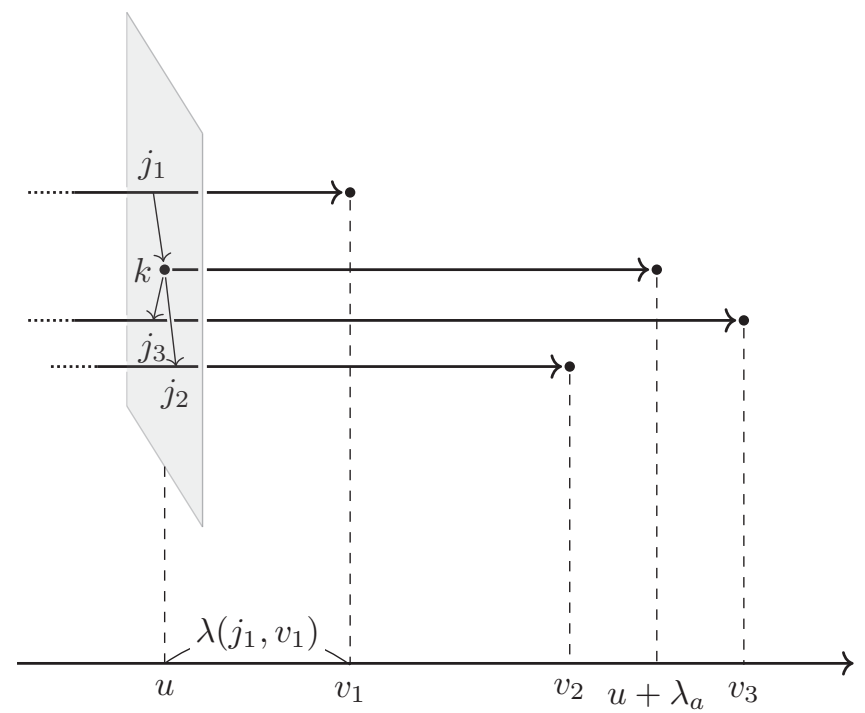

Figure 2. A schematic description of a T-system. A black point represents a mutation point. An arrow in the plane from (resp. to) the mutation point $(k, u)$ to (resp. from) a right arrow that ends at $(j, v)$ indicates that $\left[-B_{j k}(u)\right]_{+} \neq 0\left(\right.$ resp. $\left.\left[B_{j k}(u)\right]_{+} \neq 0\right)$.

Proposition 2.11 ([41, Section 5.5]). For any mutation loop $\gamma$, the family of elements $\left(Y_{a}(u)\right)_{(a, u) \in R_{\gamma}}$ satisfy the following relation in $\mathbb{P}$ for any $(a, u) \in R_{\gamma}$ :

$$
\prod_{b, p} Y_{b}(u-p)^{\check{n}_{a b ; p}^{0}}=\frac{\prod_{b, p}\left(1 \oplus Y_{b}(u-p)\right)^{\check{n}_{a b ; p}^{-}}}{\prod_{b, p}\left(1 \oplus Y_{b}(u-p)^{-1}\right)^{\check{n}_{a b ; p}^{+}}},
$$

where $\prod_{b, p}=\prod_{b=1}^{r} \prod_{p=0}^{\infty}$.

We call the family of relations in Proposition 2.11 the $Y$-system associated with $\gamma$, and the triple of matrices $\left(N_{\gamma, 0}^{\vee}, N_{\gamma,+}^{\vee}, N_{\gamma,-}^{\vee}\right)$ the $Y$-system triple of $\gamma$. From (2.11), the left-hand side in the Y-system can be rewritten as

$$
\prod_{b, p} Y_{b}(u-p)^{\check{n}_{a b ; p}^{0}}=Y_{a}(u) Y_{a^{\prime}}\left(u-\lambda_{a^{\prime}}\right) .
$$

If we define elements $P_{a}^{ \pm}(u) \in \mathbb{P}$ by

$$
P_{a}^{+}(u)=\frac{Y_{a}(u)}{1 \oplus Y_{a}(u)}, \quad P_{a}^{-}(u)=\frac{1}{1 \oplus Y_{a}(u)},
$$

the relation in Proposition 2.11 can be written in a simpler form as

$$
\prod_{b, p} P_{b}^{+}(u-p)^{\check{n}_{a b ; p}^{0}-\check{n}_{a b ; p}^{+}}=\prod_{b, p} P_{b}^{-}(u-p)^{\check{n}_{a b ; p}^{0}-\check{n}_{a b ; p}^{-}} .
$$

Figure 1 is a schematic description of a Y-system.

Next we are going to describe T-systems. Let $\gamma$ be a complete mutation loop. For any seed $(B, y, x)$, we have the following infinite length sequence of seeds:

$$
\begin{array}{lllll} 
& & \cdots & (B(-1), y(-1), x(-1)) & \stackrel{\mu_{\mathrm{i}(-1)}}{\longmapsto} \\
(B(0), y(0), x(0)) & \stackrel{\mu_{\mathrm{i}(0)}}{\longmapsto} & \cdots & (B(t-1), y(t-1), x(t-1)) & \stackrel{\mu_{\mathrm{i}(t-1)}}{\longmapsto} \\
(B(t), y(t), x(t)) & \stackrel{\mu_{\mathrm{i}(t)}}{\longmapsto} & \cdots &
\end{array}
$$


where $(B(0), y(0), x(0))=(B, y, x)$. We define $Y_{a}(u) \in \mathbb{P}$ and $T_{a}(u) \in \mathcal{F}$ for any $(a, u) \in R_{\gamma}$ by

$$
Y_{a}(u)=y_{i}(u), \quad T_{a}(u)=x_{i}(u),
$$

where $i \in I$ is a unique index such that $(i, u) \in P_{\gamma}$ and $a=\pi(i, u)$.

We define a matrix $N_{\gamma, 0}=\left(\sum_{p \in \mathbb{Z}} n_{a b ; p}^{0} z^{p}\right)_{1 \leq a, b \leq r} \in \operatorname{Mat}_{r \times r}(\mathbb{Z}[z])$ by

$$
\sum_{p \in \mathbb{Z}} n_{b a ; p}^{0} z^{p}=\delta_{a b}+\delta_{\sigma(a) b} z^{\lambda_{a}} .
$$

We also define two matrices $N_{\gamma,+}=\left(\sum_{p \in \mathbb{Z}} n_{a b ; p}^{+} z^{p}\right)_{a, b \in[1, r]}$ and $N_{\gamma,-}=\left(\sum_{p \in \mathbb{Z}} n_{a b ; p}^{-} z^{p}\right)_{a, b \in[1, r]}$ in Mat $\mathrm{M}_{r \times r}(\mathbb{Z}[z])$ by

$$
\sum_{p \in \mathbb{Z}} n_{b a ; p}^{ \pm} z^{p}=\sum_{\substack{j \in I \\ \pi(s(j, u))=b}}\left[\mp B_{j k}(u)\right]_{+} z^{\lambda(j, u)}
$$

where $(k, u) \in \pi^{-1}(a)$. The definition of $N_{ \pm}$does not depend on the choices of $(k, u)$.

Proposition 2.12 ([41, Section 5.5]). For any complete mutation loop, the family of elements $\left(Y_{a}(u)\right)_{(a, u) \in R_{\gamma}}$ and $\left(T_{a}(u)\right)_{(a, u) \in R_{\gamma}}$ satisfy the following relation in $\mathcal{F}$ for any $(a, u) \in R_{\gamma}$ :

$$
\prod_{b, p} T_{b}(u+p)^{n_{b a ; p}^{0}}=P_{a}^{+}(u) \prod_{b, p} T_{b}(u+p)^{n_{b a ; p}^{-}}+P_{a}^{-}(u) \prod_{b, p} T_{b}(u+p)^{n_{b a ; p}^{+}}
$$

where $\prod_{b, p}=\prod_{b=1}^{r} \prod_{p=0}^{\infty}$ and $P_{a}^{ \pm}(u) \in \mathbb{P}$ are defined by $(2.13)$.

We call the family of relations in Proposition 2.12 the T-system associated with $\gamma$, and the triple of matrices $\left(N_{\gamma, 0}, N_{\gamma,+}, N_{\gamma,-}\right)$ the T-system triple of $\gamma$. From (2.16), the left-hand side in the T-system can be rewritten as

$$
\prod_{b, p} T_{b}(u+p)^{n_{b a ; p}^{0}}=T_{a}(u) T_{\sigma(a)}\left(u+\lambda_{a}\right) .
$$

Figure 2 is a schematic description of a T-system.

2.3. Relation between $\mathbf{Y}$-systems and T-systems. Let $\gamma=(B, d, \mathbf{i}, \nu)$ be a mutation loop.

Lemma 2.13. The family of positive integers $\left(d_{i}(u)\right)_{(i, u) \in I \times \mathbb{Z}}$ defined by $d_{i}(u)=d_{i}$ satisfies the following:

(1) $B_{i j}(u) d_{j}(u)=-B_{j i}(u) d_{i}(u)$ for any $i, j \in I$ and $u \in \mathbb{Z}$,

(2) $d_{i}(u)=d_{j}(v)$ for any $(i, u),(j, v) \in P_{\gamma}$ such that $\pi(i, u)=\pi(j, v)$.

Proof. (1) holds since mutations preserve a symmetrizer. (2) follows from $d=\nu(d)$.

From Lemma 2.13, the positive integers $d_{1}^{\prime}, \ldots, d_{r}^{\prime}$ defined by $d_{a}^{\prime}=d_{i}(u)$ where $(i, u) \in \pi^{-1}(a)$ do not depend on the choices of $(i, u)$, and $d_{a}^{\prime}=d_{i}(u)$ for any $(i, u) \in I \times \mathbb{Z}$ such that $\pi(s(i, u))=a$. We denote by $D_{\gamma}$ the positive integer diagonal matrix $\operatorname{diag}\left(d_{1}^{\prime}, \ldots, d_{r}^{\prime}\right)$.

Proposition 2.14 (cf. Proposition 5.13, [41]). Let $\left(N_{\gamma, 0}^{\vee}, N_{\gamma,+}^{\vee}, N_{\gamma,-}^{\vee}\right)$ be the Y-system triple and $\left(N_{\gamma, 0}, N_{\gamma,+}, N_{\gamma,-}\right)$ be the T-system triple of a complete mutation loop $\gamma$. Then we have

$$
N_{\gamma, 0}^{\vee}=N_{\gamma, 0}
$$

and

$$
D_{\gamma} N_{\gamma, \varepsilon}^{\vee}=N_{\gamma, \varepsilon} D_{\gamma}
$$

for any $\varepsilon \in\{0,+,-\}$. 
Proof. The first identity follows from

$$
\sum_{p} \check{n}_{a b ; p}^{0} z^{p}=\delta_{a b}+\delta_{a^{\prime} b} z^{\lambda_{a^{\prime}}}=\delta_{b a}+\delta_{\hat{b} a} z^{\lambda_{b}}=\sum_{p} n_{a b ; p}^{0} z^{p}
$$

The second identity for $\varepsilon=0$ follows from Lemma 2.13. We now the second identity for $\varepsilon= \pm$. Let $(k, u) \in \pi^{-1}(b)$ and $\left(k^{\prime}, u^{\prime}\right) \in \pi^{-1}(a)$. Then we have

$$
\begin{aligned}
\sum_{p} \check{n}_{a b ; p}^{ \pm} z^{p}= & \sum_{\substack{(j, v) \in P_{\gamma} \\
s\left(k^{\prime}, v\right)=\left(k^{\prime}, u^{\prime}\right), \pi(j, v)=b}}\left[ \pm B_{j k^{\prime}}(v)\right]_{+} z^{\lambda(k, v)} \\
= & \sum_{\substack{j^{\prime} \in I \\
\left(s\left(j^{\prime}, u\right)\right)=a}}\left[ \pm B_{k j^{\prime}}(u)\right]_{+} z^{\lambda\left(j^{\prime}, u\right)}
\end{aligned}
$$

On the other hand, Lemma 2.13 implies that

$$
\begin{aligned}
\sum_{p} n_{a b ; p}^{ \pm} z^{p} & =\sum_{\substack { j \in I \\
\begin{subarray}{c}{s(j, u))=a\\
{ j \in I \\
\begin{subarray} { c } { s ( j , u ) ) = a \\
} }\end{subarray}}\left[\mp B_{j k}(u)\right]_{+} z^{\lambda(j, u)} \\
= & \sum_{\substack{j \in I \\
\pi(s(j, u))=a}}\left[ \pm d_{j}(u) d_{k}(u)^{-1} B_{k j}(u)\right]_{+} z^{\lambda(j, u)} \\
= & d_{a}^{\prime}\left(d_{b}^{\prime}\right)^{-1} \sum_{\substack{j \in I \\
\pi(j, u)}}\left[ \pm B_{k j}(u)\right]_{+} z^{\lambda(j, u)}
\end{aligned}
$$

For any matrix $A$, we denote the transpose of $A$ by $A^{\top}$. For any $A \in \operatorname{Mat}_{r \times r}\left(\mathbb{Z}\left[z^{ \pm 1}\right]\right)$, we define a matrix $A^{\dagger} \in \operatorname{Mat}_{r \times r}\left(\mathbb{Z}\left[z^{ \pm 1}\right]\right)$ by $A^{\dagger}=\left(\left.A\right|_{z=z^{-1}}\right)^{\top}$. Clearly, we have $\left(A^{\dagger}\right)^{\dagger}=A$ and $(A B)^{\dagger}=B^{\dagger} A^{\dagger}$ for any $A, B \in \operatorname{Mat}_{r \times r}\left(\mathbb{Z}\left[z^{ \pm 1}\right]\right)$.

Let $\left(A_{\gamma,+}^{\vee}, A_{\gamma,-}^{\vee}\right)$ and $\left(A_{\gamma,+}, A_{\gamma,-}\right)$ be the pairs of matrices defined by $A_{\gamma, \pm}^{\vee}=N_{\gamma, 0}^{\vee}-N_{\gamma, \pm}^{\vee}$ and $A_{\gamma, \pm}=$ $N_{\gamma, 0}-N_{\gamma, \pm}$, respectively. We call them the Y-system pair and the T-system pair of $\gamma$. These pairs of matrices describe the following relation between the Y-system and the T-system:

Theorem 2.15. Let $\gamma$ be a complete mutation loop, and $\left(A_{\gamma,+}^{\vee}, A_{\gamma,-}^{\vee}\right)$ and $\left(A_{\gamma,+}, A_{\gamma,-}\right)$ be the $Y$-system pair and the T-system pair of $\gamma$, respectively. Then we have

$$
A_{\gamma,+}^{\vee} A_{\gamma,-}^{\dagger}=A_{\gamma,-}^{\vee} A_{\gamma,+}^{\dagger}
$$

Proof. The claim is equivalent to the following equality:

$$
N_{0}^{\vee} N_{-}^{\dagger}-N_{0}^{\vee} N_{+}^{\dagger}-N_{-}^{\vee} N_{0}^{\dagger}+N_{+}^{\vee} N_{0}^{\dagger}=N_{+}^{\vee} N_{-}^{\dagger}-N_{-}^{\vee} N_{+}^{\dagger}
$$

Let $a, b \in[1, r]$ and $p \in \mathbb{Z}$. Let us choose an element $(i, u) \in \pi^{-1}(a)$. Let $a^{\prime}=\sigma^{-1}(a)$ and $b^{\prime}=\sigma^{-1}(b)$. Let $p_{a}=\lambda_{a^{\prime}}$ and $p_{b}=\lambda_{b^{\prime}}$. Let $v=u-p, u^{\prime}=u-p_{a}$, and $v^{\prime}=v-p_{b}$. Then the $(a b ; p)$-th entry in the 
left-hand side in (2.18) is given by

$$
\begin{aligned}
& n_{b a ;-p}^{-}+n_{b a^{\prime} ; p_{a}-p}^{-}-n_{b a ;-p}^{+}-n_{b a^{\prime} ; p_{a}-p}^{+}-\check{n}_{a b ; p}^{-}-\check{n}_{a b^{\prime} ; p+p_{b}}^{-}+\check{n}_{a b ; p}^{+}+\check{n}_{a b^{\prime} ; p+p_{b}}^{+} \\
& = \begin{cases}+n_{b a ;-p}^{-}-n_{b a ;-p}^{+}-\check{n}_{a b^{\prime} ; p+p_{b}}^{-}+\check{n}_{a b^{\prime} ; p+p_{b}}^{+} & \text {if } u \leq v \text { and } u^{\prime} \leq v^{\prime}, \\
+n_{b a-p}^{-}-n_{b a-p}^{+}+n_{b a^{\prime} ; p_{a}-p}^{-}-n_{b a^{\prime} ; p_{a}-p}^{+} & \text {if } u \leq v \text { and } v^{\prime} \leq u^{\prime}, \\
-\check{n}_{a b ; p}^{-}+\check{n}_{a b ; p}^{+}-\check{n}_{a b^{\prime} ; p+p_{b}}^{-}+\check{n}_{a b^{\prime} ; p+p_{b}}^{+} \text {if } v \leq u \text { and } u^{\prime} \leq v^{\prime}, \\
-\check{n}_{a b ; p}^{-}+\check{n}_{a b ; p}^{+}+n_{b a^{\prime} ; p_{a}-p}^{-}-n_{b a^{\prime} ; p_{a}-p}^{+} \text {if } v \leq u \text { and } v^{\prime} \leq u^{\prime},\end{cases} \\
& =\sum_{(j \in I} \begin{cases}B_{j i}(u)+B_{j i}\left(v^{\prime}\right) & \text { if } u \leq v \text { and } u^{\prime} \leq v^{\prime}, \\
B_{j i}(u)+B_{j i}\left(u^{\prime}\right) & \text { if } u \leq v \text { and } v^{\prime} \leq u^{\prime}, \\
B_{j i}(v)+B_{j i}\left(v^{\prime}\right) & \text { if } v \leq u \text { and } u^{\prime} \leq v^{\prime}, \\
B_{j i}(v)+B_{j i}\left(u^{\prime}\right) & \text { if } v \leq u \text { and } v^{\prime} \leq u^{\prime},\end{cases} \\
& =\sum_{j \in I, \pi(j, v)=b}\left(B_{j i}(\min (u, v))+B_{j i}\left(\max \left(u^{\prime}, v^{\prime}\right)\right)\right) .
\end{aligned}
$$

On the other hand, the $(a b ; p)$-th entry in the right-hand side in $(2.18)$ is given by

$$
\begin{aligned}
& \sum_{c=1}^{r} \sum_{w \in \mathbb{Z}}\left(\check{n}_{a c ; u-w}^{+} n_{b c ; v-w}^{-}-\check{n}_{a c ; u-w}^{-} n_{b c ; v-w}^{+}\right) \\
& =\sum_{\substack{j \in I,(k, w) \in P_{\gamma} \\
(j, v) \in P_{\gamma}, \pi(j, v)=b \\
\max \left(u^{\prime}, v^{\prime}\right)<w<\min (u, v)}}\left(\left[B_{k i}(w)\right]_{+}\left[B_{j k}(w)\right]_{+}-\left[-B_{j k}(w)\right]_{+}\left[-B_{k i}(w)\right]_{+}\right) .
\end{aligned}
$$

These two entries coincide since

$$
=\sum_{\substack{(k, w) \in P_{\gamma} \\ B_{j i}(\min (u, v))+B_{j i}\left(\max \left(u^{\prime}, v^{\prime}\right)\right)}}\left(\left[B_{k i}(w)\right]_{+}\left[B_{j k}(w)\right]_{+}-\left[-B_{j k}(w)\right]_{+}\left[-B_{k i}(w)\right]_{+}\right)
$$

by the rule of matrix mutations (2.1).

\section{Axiomatic approach to Y/T systems}

3.1. T-data. Let $r$ be a positive integer. As in the last section, we define an involution $\dagger: \operatorname{Mat}_{r \times r}(\mathbb{Q}(z)) \rightarrow$ Mat $_{r \times r}(\mathbb{Q}(z))$ by $A^{\dagger}=\left(\left.A\right|_{z=z^{-1}}\right)^{\top}$.

For a triple $\left(N_{0}, N_{+}, N_{-}\right)$of the matrices in $\operatorname{Mat}_{r \times r}(\mathbb{Z}[z])$ whose entries are given by

$$
N_{\varepsilon}=\left(\sum_{p \in \mathbb{Z} \geq 0} n_{a b ; p}^{\varepsilon} z^{p}\right)_{a, b \in[1, r]}
$$

we consider the following conditions:

(N1) $n_{a b ; p}^{0}=\delta_{a b} \delta_{p 0}+\delta_{a \sigma(b)} \delta_{p p_{a}}$ for some $\sigma \in \mathfrak{S}_{r}$ and $p_{a} \in \mathbb{Z}_{>0}$,

(N2) $n_{a b ; p}^{+} \geq 0$ and $n_{a b ; p}^{-} \geq 0$ for any $a, b, p$,

(N3) $n_{a b ; p}^{+}=0$ and $n_{a b ; p}^{-}=0$ unless $0<p<p_{a}$,

(N4) $n_{a b ; p}^{+} n_{a b ; p}^{-}=0$ for any $a, b, p$.

Definition 3.1. We say that a triple of matrices $\alpha=\left(A_{+}, A_{-}, D\right)$ is a T-datum of size $r$ if $A_{ \pm}$can be written as $A_{ \pm}=N_{0}-N_{ \pm}$by a triple of matrices $\left(N_{0}, N_{+} N_{-}\right)$in $\operatorname{Mat}_{r \times r}(\mathbb{Z}[z])$ satisfying (N1)-(N4), and $D$ is a positive integer diagonal matrix that satisfies the following conditions:

- $N_{0} D=D N_{0}$, 
- $D^{-1} N_{ \pm} D \in \operatorname{Mat}_{r \times r}(\mathbb{Z}[z])$,

- $A_{+} D A_{-}^{\dagger}=A_{-} D A_{+}^{\dagger}$.

It is clear that the triple $\left(N_{0}, N_{+}, N_{-}\right)$that satisfies the conditions (N1)-(N4) is uniquely recovered from $\left(A_{+}, A_{-}\right)$as follows: $N_{0}=\left[A_{+}\right]_{+}=\left[A_{-}\right]_{+}, N_{+}=\left[-A_{+}\right]_{+}$, and $N_{-}=\left[-A_{-}\right]_{+}$, where we take [ $]_{+}$for each coefficient. Note that both matrices $A_{+}$and $A_{-}$have non-zero determinants since their determinants are monic polynomials with constant terms 1, which follows from the conditions (N1) and (N3).

We say that the last equation

$$
A_{+} D A_{-}^{\dagger}=A_{-} D A_{+}^{\dagger}
$$

in Definition 3.1 is the symplectic relation due to the following lemma, which can be easily verified:

Lemma 3.2. Let $A_{+}, A_{-} \in \operatorname{Mat}_{r \times r}\left(\mathbb{Z}\left[z^{ \pm 1}\right]\right)$ be matrices with non-zero determinants, and $D$ be a positive integer diagonal matrix. Then the following conditions are equivalent:

(1) $A_{+} D A_{-}^{\dagger}=A_{-} D A_{+}^{\dagger}$.

(2) $A_{+} D A_{-}^{\dagger}$ is a $\dagger$-invariant.

(3) $A_{-} D A_{+}^{\dagger}$ is a †-invariant.

(4) $\left(A_{-}\right)^{-1} A_{+} D$ is a $\dagger$-invariant.

(5) $\left(A_{+}\right)^{-1} A_{-} D$ is a †-invariant.

(6) $D^{-1}\left(A_{-}\right)^{-1} A_{+}$is a †-invariant.

(7) $D^{-1}\left(A_{+}\right)^{-1} A_{-}$is a $\dagger$-invariant.

(8) The rows of the $r \times 2 r$ matrices $\left[A_{+} A_{-}\right]$are pairwise orthogonal with respect to the symplectic pairing $\langle\rangle:,\left(\mathbb{Z}\left[z^{ \pm 1}\right]\right)^{2 r} \times\left(\mathbb{Z}\left[z^{ \pm 1}\right]\right)^{2 r} \rightarrow \mathbb{Z}\left[z^{ \pm 1}\right]$ defined by

$$
\left\langle\left[\begin{array}{l}
f(z) \\
g(z)
\end{array}\right],\left[\begin{array}{l}
f^{\prime}(z) \\
g^{\prime}(z)
\end{array}\right]\right\rangle=\left[\begin{array}{ll}
f(z)^{\top} & g(z)^{\top}
\end{array}\right]\left[\begin{array}{cc}
O & D \\
-D & O
\end{array}\right]\left[\begin{array}{l}
f^{\prime}\left(z^{-1}\right) \\
g^{\prime}\left(z^{-1}\right)
\end{array}\right],
$$

where $f(z), g(z), f^{\prime}(z), g^{\prime}(z) \in\left(\mathbb{Z}\left[z^{ \pm 1}\right]\right)^{r}$.

Let $\alpha=\left(A_{+}, A_{-}, D\right)$ be a T-datum. Then the triple $\alpha^{\vee}=\left(A_{+}^{\vee}, A_{-}^{\vee}, D^{\vee}\right)$ defined by $N_{\varepsilon}^{\vee}=D^{-1} N_{\varepsilon} D$ and $D^{\vee}=\delta D^{-1}$ where $\delta$ is the product of the greatest common divisor and the least common multiple of all the entries in $D$, is also a T-datum. The T-datum $\alpha^{\vee}$ is called the Langlands dual of $\alpha$. Clearly, we have $\alpha^{\vee \vee}=\alpha$. We write the entries of $N_{\varepsilon}^{\vee}$ as

$$
N_{\varepsilon}^{\vee}=\left(\sum_{p \in \mathbb{Z} \geq 0} \check{n}_{a b ; p}^{\varepsilon} z^{p}\right)_{a, b \in[1, r]} .
$$

Definition 3.3. Let $\alpha=\left(A_{+}, A_{-}, D\right)$ be a T-datum of size $r$. We say that a subset $R \subseteq[1, r] \times \mathbb{Z}$ is consistent for $\alpha$ if the following conditions are satisfied:

(R1) If $(a, u) \in R$ and $n_{a b ; p}^{0}, n_{a b ; p}^{+}$, or $n_{a b ; p}^{-} \neq 0$, then $(b, u-p) \in R$.

(R2) If $(a, u) \in R$ and $n_{b a ; p}^{0}, n_{b a ; p}^{+}$, or $n_{b a ; p}^{-} \neq 0$, then $(b, u+p) \in R$.

(R3) There exists a positive integer $t$ such that $R=R^{(t)}$ and

$$
[1, r] \times \mathbb{Z}=\bigsqcup_{k=0}^{t-1} R^{(k)}
$$

where $R^{(k)}:=\{(a, u+k) \mid(a, u) \in R\}$.

For example, $[1, r] \times \mathbb{Z}$ itself is always consistent since (R1) and (R2) are obvious, and (R3) is satisfied by setting $t=1$. Note that the positive integer $t$ in (R3) is uniquely determined from $R$. In the conditions (R1) and (R2), we can replace $n$ with $\check{n}$. From (N1) together with (R1) and (R2), we have

$$
\begin{array}{rll}
(a, u) \in R & \text { if and only if } & \left(\sigma(a), u+p_{\sigma(a)}\right) \in R \\
& \text { if and only if } & \left(\sigma^{-1}(a), u-p_{a}\right) \in R .
\end{array}
$$


Definition 3.4. Let $(\alpha, R)$ and $\left(\alpha^{\prime}, R^{\prime}\right)$ be pairs of T-data of size $r$ and consistent subsets for them. They are called equivalent if there exists a permutation $\rho \in \mathfrak{S}_{r}$ such that $A_{ \pm}^{\prime}=\rho\left(A_{ \pm}\right), D^{\prime}=\rho(D)$, and $R^{\prime}=\rho(R)$, where $\rho(R)=\{(\rho(a), u) \mid(a, u) \in R\}$.

Definition 3.5. Let $\alpha$ be a T-datum of size $r$, and $R \subseteq[1, r] \times \mathbb{Z}$ be a consistent subset for $\alpha$. We say that a family of elements $\left(Y_{a}(u)\right)_{(a, u) \in R}$ is a solution of the $Y$-system associated with $(\alpha, R)$ in a semifield $\mathbb{P}$ if $Y_{a}(u) \in \mathbb{P}$ and the following relation holds in $\mathbb{P}$ for any $(a, u) \in R$ :

$$
\prod_{b, p} Y_{b}(u-p)^{\check{n}_{a b ; p}^{0}}=\frac{\prod_{b, p}\left(1 \oplus Y_{b}(u-p)\right)^{\check{n}_{a b ; p}^{-}}}{\prod_{b, p}\left(1 \oplus Y_{b}(u-p)^{-1}\right)^{\check{n}_{a b ; p}^{+}}},
$$

where $\prod_{b, p}=\prod_{b=1}^{r} \prod_{p=0}^{\infty}$.

For any solution of the Y-system associated with $(\alpha, R)$ in $\mathbb{P}$, we define elements $P_{a}^{ \pm}(u) \in \mathbb{P}((a, u) \in R)$ by

$$
P_{a}^{+}(u)=\frac{Y_{a}(u)}{1 \oplus Y_{a}(u)}, \quad P_{a}^{-}(u)=\frac{1}{1 \oplus Y_{a}(u)} .
$$

Definition 3.6. Let $\alpha$ be a T-datum of size $r$, and $R \subseteq[1, r] \times \mathbb{Z}$ be a consistent subset for $\alpha$. Let $Y=\left(Y_{a}(u)\right)_{(a, u) \in R}$ be a solution of the Y-system associated with $(\alpha, R)$ in a semifield $\mathbb{P}$. Let $\mathcal{T}(\alpha, R, Y)$ be the $\mathbb{Z P}$-algebra generated by the indeterminates $\left(T_{a}(u)^{ \pm 1}\right)_{(a, u) \in R}$ subject to the relation

$$
\prod_{b, p} T_{b}(u+p)^{n_{b a ; p}^{0}}=P_{a}^{+}(u) \prod_{b, p} T_{b}(u+p)^{n_{b a ; p}^{-}}+P_{a}^{-}(u) \prod_{b, p} T_{b}(u+p)^{n_{b a ; p}^{+}}
$$

for any $(a, u) \in R$, together with $T_{a}(u) T_{a}(u)^{-1}=1$. We define $\mathcal{T}^{\circ}(\alpha, R, Y)$ to be the subalgebra of $\mathcal{T}(\alpha, R, Y)$ generated by $\left(T_{a}(u)\right)_{(a, u) \in R}$. We say that $\mathcal{T}^{\circ}(\alpha, R, Y)$ is the $T$-algebra associated with $(\alpha, R, Y)$. We often denote $\mathcal{T}^{\circ}(\alpha, R, Y)$ by $\mathcal{T}^{\circ}(\alpha)$ when $R=[1, r] \times \mathbb{Z}$ and $\mathbb{P}$ is the trivial semifield.

The family of relations (3.4) is called the $Y$-system associated with $(\alpha, R)$, and the family of relations (3.5) is called the T-system associated with $(\alpha, R, Y)$.

Example 3.7 (Somos-4 recurrence). The triple of $1 \times 1$ matrices $\alpha=\left(A_{+}, A_{-}, D\right)$ defined by

$$
A_{+}=\left[1-2 z^{2}+z^{4}\right], \quad A_{-}=\left[1-z-z^{3}+z^{4}\right], \quad D=[1]
$$

is a T-datum, and the whole set $R=\{1\} \times \mathbb{Z}$ is consistent for $\alpha$. The family $Y=(Y(u))_{(1, u) \in R}$ defined by $Y(u)=c_{1} c_{2}^{-1}$ for any $u \in \mathbb{Z}$ is a solution of the Y-system associated with $\alpha$ in $\operatorname{Trop}\left(c_{1}, c_{2}\right)$, where we denote $Y_{1}(u)$ by $Y(u)$. The family of relations

$$
T(u) T(u+4)=c_{1} T(u+1) T(u+3)+c_{2} T(u+2)^{2}
$$

for $u \in \mathbb{Z}$ is the T-system associated with $(\alpha, R, Y)$, where we denote $T_{1}(u)$ by $T(u)$. This is called the Somos-4 recurrence [12].

Example 3.8 (Bipartite belt). Let $A=\left(2 \delta_{a b}-n_{a b}\right)_{a, b \in[1, r]}$ be a symmetrizable generalized Cartan matrix, and $D$ be a right symmetrizer of $A$. Suppose that $A$ is bipartite, that is, there exists a function $\epsilon:[1, r] \rightarrow\{1,-1\}$ such that $n_{a b}>0$ implies $\epsilon(a) \neq \epsilon(b)$ for any $a, b \in[1, r]$. Let $N=2 I_{r}-A$. Then the triple of $r \times r$ matrices $\alpha=\left(A_{+}, A_{-}, D\right)$ defined by

$$
A_{+}=\left(1+z^{2}\right) I_{r}, \quad A_{-}=\left(1+z^{2}\right) I_{r}-z N
$$

is a T-datum since

$$
A_{+} D A_{-}^{\dagger}-A_{-} D A_{+}^{\dagger}=\left(z+z^{-1}\right)\left(-D N^{\top}+N D\right)=0,
$$

and the set $R \subseteq[1, r] \times \mathbb{Z}$ defined by

$$
R=\left\{(a, u) \in[1, r] \times \mathbb{Z} \mid \epsilon(a)=(-1)^{u-1}\right\}
$$




\begin{tabular}{|c|c|c|c|c|c|c|c|}
\hline$u$ & 0 & 1 & 2 & 3 & 4 & 5 & 6 \\
\hline$Y_{1}(u)$ & $y_{1}$ & & $\underline{1 \oplus y_{2} \oplus y_{1} y_{2}}$ & & 1 & & $\left(1 \oplus y_{1}\right) y_{2}$ \\
\hline$Y(1)$ & & & $y_{1}$ & $1 \oplus y_{2}$ & $y_{2}$ & & \\
\hline$I_{2}(u)$ & & $\left(1 \oplus y_{1}\right) y_{2}$ & & $y_{1} y_{2}$ & & $y_{1}$ & \\
\hline$T_{1}(u)$ & $x_{1}$ & & $y_{1} x_{2}+1$ & & $x_{1}+y_{2}$ & & $x_{2}$ \\
\hline$T_{2}(u)$ & & $x_{2}$ & $\left(1 \oplus y_{1}\right) x_{1}$ & $\frac{y_{1} y_{2} x_{2}+x_{1}+y_{2}}{\left(1 \oplus y_{2} \oplus y_{1} y_{2}\right) x_{1} x_{2}}$ & $\left(1 \oplus y_{2}\right) x_{2}$ & $x_{1}$ & \\
\hline
\end{tabular}

TABLE 1. The bipartite belt associated with the Cartan matrix of type $A_{2}$.

is consistent for $\alpha$. The family of relations

$$
Y_{a}(u) Y_{a}(u-2)=\prod_{b=1}^{r}\left(1 \oplus Y_{b}(u-1)\right)^{n_{b a}}
$$

for $(a, u) \in R$ is the $\mathrm{Y}$-system associated with $\alpha$, and

$$
T_{a}(u) T_{a}(u+2)=\frac{Y_{a}(u) \prod_{b=1}^{r} T_{b}(u+1)^{n_{b a}}+1}{1 \oplus Y_{a}(u)}
$$

for $(a, u) \in R$ is the T-system associated with $(\alpha, R, Y)$. The discrete dynamical system given by these relations are called the bipartite belt associated with $A[14$, Section 8$]$.

If $A$ is the Cartan matrix of type $A_{2}$ for instance, the triple of matrices in $\alpha$ is given by

$$
A_{+}=\left[\begin{array}{cc}
1+z^{2} & 0 \\
0 & 1+z^{2}
\end{array}\right], \quad A_{-}=\left[\begin{array}{cc}
1+z^{2} & -z \\
-z & 1+z^{2}
\end{array}\right], \quad D=\left[\begin{array}{ll}
1 & 0 \\
0 & 1
\end{array}\right]
$$

Table 1 is the bipartite belt associated with the Cartan matrix of type $A_{2}$ with $\epsilon(1)=-1$ and $\epsilon(2)=1$, where $y_{1}$ and $y_{2}$ are arbitrary elements in the underlying semifield $\mathbb{P}$, and we write $T_{1}(0)$ and $T_{2}(1)$ as $x_{1}$ and $x_{2}$, respectively.

3.2. T-data from mutation loops. Let us see that we can obtain T-data from mutation loops. Let $\gamma$ be a complete mutation loop of length $r$. Let $\left(N_{\gamma, 0}, N_{\gamma,+}, N_{\gamma,-}\right)$ be the T-system triple and $\left(A_{\gamma,+}, A_{\gamma,-}\right)$ be the T-system pair of $\gamma$, which are defined in Section 2.2. Let $D_{\gamma}$ be the positive integer diagonal matrix in Proposition 2.14.

Lemma 3.9. The triple $\left(N_{\gamma, 0}, N_{\gamma,+}, N_{\gamma,-}\right)$ satisfies the conditions (N1)-(N4).

Proof. The condition (N1) is satisfied if $p_{a}=\lambda_{\sigma^{-1}(a)}$ and $\sigma$ is as in (2.8). The condition (N2) is obvious from the definition. The definition (2.17) implies (N3) since $0<\lambda(j, u)<\lambda_{\sigma^{-1}(b)}$ if $\pi(s(j, u))=b$ and $(j, u) \notin P_{\gamma}$. The definition (2.17) also implies (N4) since at least one of $[b]_{+}$and $[-b]_{+}$is zero for any integer $b$.

Proposition 3.10. The triple $\alpha_{\gamma}:=\left(A_{\gamma,+}, A_{\gamma,-}, D_{\gamma}\right)$ is a T-datum.

Proof. We have $D_{\gamma} N_{\gamma, 0}=D_{\gamma} N_{\gamma, 0}^{\vee}=N_{\gamma, 0} D_{\gamma}$, and $D_{\gamma}^{-1} N_{\gamma, \pm} D_{\gamma}=N_{\gamma, \pm}^{\vee} \in \operatorname{Mat}_{r \times r}(\mathbb{Z}[z])$ by Proposition 2.14. We also have

$$
\begin{aligned}
A_{\gamma,+} D_{\gamma} A_{\gamma,-}^{\dagger}-A_{\gamma,-} D_{\gamma} A_{\gamma,+}^{\dagger} & =D_{\gamma} A_{\gamma,+}^{\vee} D_{\gamma}^{-1} D_{\gamma} A_{\gamma,-}^{\dagger}-D_{\gamma} A_{\gamma,-}^{\vee} D_{\gamma}^{-1} D_{\gamma} A_{\gamma,+}^{\dagger} \\
& =D_{\gamma}\left(A_{\gamma,+}^{\vee} A_{\gamma,-}^{\dagger}-A_{\gamma,-}^{\vee} A_{\gamma,+}^{\dagger}\right) \\
& =0
\end{aligned}
$$


by Proposition 2.14 and Theorem 2.15 .

Let $D_{\gamma}^{\vee}$ the diagonal matrix defined by $D_{\gamma}^{\vee}=\delta D_{\gamma}^{-1}$ where $\delta$ is the product of the greatest common divisor and the least common multiple of all the entries in $D_{\gamma}$.

Corollary 3.11. The triple $\alpha_{\gamma}^{\vee}:=\left(A_{\gamma,+}^{\vee}, A_{\gamma,-}^{\vee}, D_{\gamma,-}^{\vee}\right)$ is a T-datum, and it is the Langlands dual of $\alpha_{\gamma}$.

Proposition 3.12. The subset $R_{\gamma} \subseteq[1, r] \times \mathbb{Z}$ defined in (2.7) is consistent for $\alpha_{\gamma}$.

Proof. The conditions (R1) and (R2) follow from Proposition 2.11 and 2.12, respectively. The condition (R3) is satisfied if we define $t$ in (R3) as the length of the partition of $\mathbf{i}$.

3.3. Mutation loops from T-data. In this section, we prove all T-data can be obtained from mutation loops up to equivalence.

Theorem 3.13. Suppose that $\alpha=\left(A_{+}, A_{-}, D\right)$ is a $T$-datum of size $r$, and $R \subseteq[1, r] \times \mathbb{Z}$ is consistent for $\alpha$. Then there exists a complete mutation loop $\gamma$ of length $r$ such that $\left(\alpha_{\gamma}, R_{\gamma}\right)$ and $(\alpha, R)$ are equivalent, where $\alpha_{\gamma}=\left(A_{\gamma,+}, A_{\gamma,-}, D_{\gamma}\right)$.

The rest of Section 3.3 is devoted to the proof of Theorem 3.13. Let $p_{1}, \ldots, p_{r}$ be positive integers and $\sigma$ be the permutation of $[1, r]$ in (N1). Let $\psi:[1, r] \times \mathbb{Z} \rightarrow[1, r] \times \mathbb{Z}$ be the bijection defined by $\psi(a, u)=(a, u+1)$. We define a family of subsets $R^{(k)}(k \in \mathbb{Z})$ by $R^{(k)}=\psi^{k}(R)$ as in Definition 3.3. We also define a subset $R^{(k)}(u) \subseteq R^{(k)}$ for any $u \in \mathbb{Z}$ by $R^{(k)}(u)=\left\{(a, u+p) \in R^{(k)} \mid 0 \leq p<p_{a}\right\}$. We denote $R^{(0)}(u)$ by $R(u)$. The map $\psi$ restricts to a bijection $\left.\psi\right|_{R^{(k)}(u)}: R^{(k)}(u) \rightarrow R^{(k+1)}(u+1)$. We will write this restriction simply $\psi$ when no confusion can arise. Let $t$ be the integer in (R3) in Definition 3.3. Then we have $R^{(k)}(u)=R^{(k+t)}(u)$. In particular, the map $\psi^{t}$ restricts to a bijection $\left.\psi^{t}\right|_{R^{(k)}(u)}: R^{(k)}(u) \rightarrow R^{(k)}(u+t)$. We also define a family of bijections $\varphi_{u}: R(u) \rightarrow R(u+1)(u \in \mathbb{Z})$ by

$$
\varphi_{u}(a, u+p)= \begin{cases}(a, u+p) & \text { if } p \neq 0 \\ \left(\sigma(a), u+p_{\sigma(a)}\right) & \text { if } p=0 .\end{cases}
$$

It is easy to check that $\psi^{t}$ and $\varphi$ commute in the sense that $\psi^{t} \circ \varphi_{u}=\varphi_{u+t} \circ \psi^{t}$. We define $R_{0}(u) \subseteq R(u)$ by $R_{0}(u)=\{(a, u+p) \in R(u) \mid p=0\}$, which is endowed with the linear order coming from the standard linear order on $[1, r]$.

For any $u \in \mathbb{Z}$, we define an $R(u) \times R(u)$ matrix $\bar{B}(u)$ by

$$
\begin{gathered}
\bar{B}_{(a, u+p)(b, u+q)}(u)=-n_{a b ; p-q}^{+}+n_{a b ; p-q}^{-}+\check{n}_{b a ; q-p}^{+}-\check{n}_{b a ; q-p}^{-} \\
+\sum_{c=1}^{r} \sum_{v=0}^{\min (p, q)}\left(n_{a c ; p-v}^{+} \check{n}_{b c ; q-v}^{-}-n_{a c ; p-v}^{-} \check{n}_{b c ; q-v}^{+}\right)
\end{gathered}
$$

where $n^{ \pm}$and $\check{n}^{ \pm}$are defined in (3.1) and (3.3), respectively. Note that $\bar{B}(u)$ and $\bar{B}(v)$ may be different matrices if $u \neq v$, even though they have the same expression (3.7). Rather, these matrices are related by mutations, as the following lemma shows:

Lemma 3.14. $\bar{B}(u+1)=\varphi_{u}\left(\mu_{R_{0}(u)}(\bar{B}(u))\right)$ for any $u \in \mathbb{Z}$.

Proof. Let $\bar{B}^{\prime}(u)=\mu_{R_{0}(u)}(\bar{B}(u))$ and $(a, u+p),(b, u+q) \in R(u)$. Then we have

$$
\bar{B}_{(a, u+p)(b, u+q)}^{\prime}(u)= \begin{cases}-\bar{B}_{(a, u+p)(b, u+q)}(u) & \text { if } p \text { or } q=0, \\ \bar{B}_{(a, u+p)(b, u+q)}(u)-\sum_{c=1}^{r}\left(n_{a c ; p}^{+} \check{n}_{b c ; q}^{-}-n_{a c ; p}^{-} \check{n}_{b c ; q}^{+}\right) & \text {if } p, q>0\end{cases}
$$


since

$$
\begin{aligned}
& \sum_{\begin{array}{c}
c \in[1, r] \\
(c, u) \in R_{0}(u)
\end{array}}\left(\left[\bar{B}_{(a, u+p)(c, u)}(u)\right]_{+}\left[\bar{B}_{(c, u)(b, u+q)}(u)\right]_{+}\right. \\
& \left.\quad-\left[-\bar{B}_{(a, u+p)(c, u)}(u)\right]_{+}\left[-\bar{B}_{(c, u)(b, u+q)}(u)\right]_{+}\right) \\
& =\sum_{c=1}^{r}\left(n_{a c ; p}^{-} \check{n}_{b c ; q}^{+}-n_{a c ; p}^{+} \check{n}_{b c ; q}^{-}\right)
\end{aligned}
$$

by (N2), (N4), and (R1).

The proof is divided into the following cases: (i) $p, q>0$, (ii) $p=0$ and $q>0$, (iii) $p>0$ and $q=0$, and (iv) $p=q=0$. For the case (i), we have

$$
\bar{B}_{(a, u+p)(b, u+q)}(u)-\bar{B}_{(a, u+p)(b, u+q)}(u+1)=\sum_{c=1}^{r}\left(n_{a c ; p}^{+} \check{n}_{b c ; q}^{-}-n_{a c ; p}^{-} \check{n}_{b c ; q}^{+}\right)
$$

by (3.7), and this yields the desired equality since $\varphi_{u}(a, u+p)=(a, u+p)$ and $\varphi_{u}(b, u+q)=(b, u+q)$. For the case (ii), we have $\varphi_{u}(a, u)=\left(\hat{a}, u+p_{\hat{a}}\right)$ where $\hat{a}=\sigma(a)$. Then we have

$$
\bar{B}_{(a, u)(b, u+q)}^{\prime}(u)=-\bar{B}_{(a, u)(b, u+q)}(u)=-\check{n}_{b a ; q}^{+}+\check{n}_{b a ; q}^{-}
$$

and

$$
\begin{gathered}
\bar{B}_{\left(\hat{a}, u+p_{\hat{a}}\right)(b, u+q)}(u+1)=-n_{\hat{a} b ; p_{\hat{a}}-q}^{+}+n_{\hat{a} b ; p_{\hat{a}}-q}^{-}+\check{n}_{b \hat{a} ; q-p_{\hat{a}}}^{+}-\check{n}_{b \hat{a} ; q-p_{\hat{a}}}^{-} \\
+\sum_{c=1}^{r} \sum_{v=1}^{\min \left(p_{\hat{a}}, q\right)}\left(n_{\hat{a} c ; p_{\hat{a}}-v}^{+} \check{n}_{b c ; q-v}^{-}-n_{\hat{a} c ; p_{\hat{a}}-v}^{-} \check{n}_{b c ; q-v}^{+}\right) .
\end{gathered}
$$

These coincide by the $\left(b \hat{a} ; q-p_{\hat{a}}\right)$-th entry in the symplectic relation, together with (N1) and (N3). For the case (iii), we have $\varphi_{u}(b, u)=\left(\hat{b}, u+p_{\hat{b}}\right)$ where $\hat{b}=\sigma(b)$. Then we have

and

$$
\bar{B}_{(a, u+p)(b, u)}^{\prime}(u)=-\bar{B}_{(a, u+p)(b, u)}(u)=n_{a b ; p}^{+}-n_{a b ; p}^{-}
$$

$$
\begin{gathered}
\bar{B}_{(a, u+p)\left(\hat{b}, u+p_{\hat{b}}\right)}(u+1)=-n_{a \hat{b} ; p-p_{\hat{b}}}^{+}+n_{a \hat{b} ; p-p_{\hat{b}}}^{-}+\check{n}_{\hat{b} a ; p_{\hat{b}}-p}^{+}-\check{n}_{\hat{b} a ; p_{\hat{b}}-p}^{-} \\
+\sum_{c=1}^{r} \sum_{v=1}^{\min \left(p, p_{\hat{b}}\right)}\left(n_{a c ; p-v}^{+} \check{n}_{\hat{b} c ; p_{\hat{b}}-v}^{-}-n_{a c ; p-v}^{-} \check{n}_{\hat{b} c ; p_{\hat{b}}-v}^{+}\right)
\end{gathered}
$$

These coincide by the $\left(a \hat{b} ; p-p_{\hat{b}}\right)$-th entry in the symplectic relation, together with (N1) and (N3). For the case (iv), we have $\varphi_{u}(a, u)=\left(\hat{a}, u+p_{\hat{a}}\right)$ and $\varphi_{u}(b, u)=\left(\hat{b}, u+p_{\hat{b}}\right)$. Then we have

$$
\bar{B}_{(a, u)(b, u)}^{\prime}(u)=-\bar{B}_{(a, u)(b, u)}(u)=0 .
$$

On the other hand, we have

$$
\begin{gathered}
\bar{B}_{\left(\hat{a}, u+p_{\hat{a}}\right)\left(\hat{b}, u+p_{\hat{b}}\right)}(u+1)=-n_{\hat{a} \hat{b} ; p_{\hat{a}}-p_{\hat{b}}}^{+}+n_{\hat{a} \hat{b} ; p_{\hat{a}}-p_{\hat{b}}}^{-}+\check{n}_{\hat{b} \hat{a} ; p_{\hat{b}}-p_{\hat{a}}}^{+}-\check{n}_{\hat{b} \hat{a} ; p_{\hat{b}}-p_{\hat{a}}}^{-} \\
+\sum_{c=1}^{r} \sum_{v=1}^{\min \left(p_{\hat{a}}, p_{\hat{b}}\right)}\left(n_{\hat{a} c ; p_{\hat{a}}-v}^{+} \check{n}_{\hat{b} c ; p_{\hat{b}}-v}^{-}-n_{\hat{a} c ; p_{\hat{a}}-v}^{-} \check{n}_{\hat{b} c ; p_{\hat{b}}-v}^{+}\right)=0
\end{gathered}
$$

by the $\left(\hat{a} \hat{b} ; p_{\hat{a}}-p_{\hat{b}}\right)$-th entry in the symplectic relation, together with (N1) and (N3).

Lemma 3.15. $\bar{B}(u+t)=\psi^{t}(\bar{B}(u))$ for any $u \in \mathbb{Z}$.

Proof. Since $R(u)=R(u+t)$, the lemma follows from the fact that $\bar{B}(u+t)$ and $\bar{B}(u)$ have the same expression (3.7). 
We define an index set $I$ by $I=R(0)$, and define an $I \times I$ integer matrices $B$ by $B=\bar{B}(0)$, that is, $B=\left(B_{(a, p)(b, q)}\right)_{(a, p),(b, q) \in R(0)}$ and

$$
\begin{array}{r}
B_{(a, p)(b, q)}=-n_{a b ; p-q}^{+}+n_{a b ; p-q}^{-}+\check{n}_{b a ; q-p}^{+}-\check{n}_{b a ; q-p}^{-} \\
+\sum_{c=1}^{r} \sum_{v=0}^{\min (p, q)}\left(n_{a c ; p-v}^{+} \check{n}_{b c ; q-v}^{-}-n_{a c ; p-v}^{-} \check{n}_{b c ; q-v}^{+}\right) .
\end{array}
$$

We define a tuple of positive integer $d=\left(d_{a, u}\right)_{(a, u) \in R(u)}$ by $d_{a, u}=d_{a}$, where $d_{a}$ is the $a$-th entry in $D$. We also define $\mathbf{i}=\mathbf{i}(0)|\cdots| \mathbf{i}(t-1)$ by $\mathbf{i}(u)=\left(\varphi_{u-1} \circ \cdots \circ \varphi_{0}\right)^{-1}\left(R_{0}(u)\right)$, where each $\mathbf{i}(u)$ is endowed with the linear order coming from the linear order on $R_{0}(u)$. Finally, we define $\nu=\left(\varphi_{t-1} \circ \cdots \circ \varphi_{0}\right)^{-1} \circ \psi^{t}$.

Lemma 3.16. $\gamma=(B, d, \mathbf{i}, \nu)$ is a complete mutation loop of length $r$.

Proof. It is easy to check that $B$ is a skew-symmetrizable matrix with the symmetrizer $d$. Let us denote by $\vec{\varphi}_{u}$ the composition $\varphi_{u-1} \circ \cdots \circ \varphi_{0}$. We now prove

$$
\left(\mu_{\mathbf{i}(u-1)} \circ \cdots \circ \mu_{\mathbf{i}(0)}\right)(B)=\left(\vec{\varphi}_{u}\right)^{-1}(\bar{B}(u))
$$

for any $u=0, \ldots, t-1$ by induction on $u$. The equation (3.10) holds when $u=0$ since $B=\bar{B}(0)$ by definition. Suppose that $u>0$. By the induction hypothesis and Lemma 3.14, we have

$$
\begin{aligned}
& \left(\mu_{\mathbf{i}(u-1)} \circ \mu_{\mathbf{i}(u-2)} \circ \cdots \circ \mu_{\mathbf{i}(0)}\right)(B) \\
= & \left(\mu_{\mathbf{i}(u-1)} \circ\left(\vec{\varphi}_{u-1}\right)^{-1}\right)(\bar{B}(u-1)) \\
= & \left(\left(\vec{\varphi}_{u-1}\right)^{-1} \circ \mu_{R_{0}(u)}\right)(\bar{B}(u-1)) \\
= & \left(\left(\vec{\varphi}_{u-1}\right)^{-1} \circ\left(\varphi_{u-1}\right)^{-1}\right)(\bar{B}(u)) \\
= & \left(\vec{\varphi}_{u}\right)^{-1}(\bar{B}(u)),
\end{aligned}
$$

and (3.10) is proved. Applying (3.10) for $u=t-1$ yields

$$
\mu_{\mathbf{i}}(B)=\left(\vec{\varphi}_{t}\right)^{-1}(\bar{B}(t))=\left(\left(\vec{\varphi}_{t}\right)^{-1} \circ \psi^{t}\right)(B) .
$$

This shows that $\gamma$ is a mutation loop. By (R3) in Definition 3.3, we have

$$
\{(a, 0) \mid a \in[1, r]\}=\bigsqcup_{u=0}^{t-1} \psi^{-u}\left(R_{0}(u)\right)
$$

as a set. This implies that the length of $\gamma$ is $r$. The completeness follows from the fact that the latency of $((a, p), 0) \in I \times\{0\}$ is $p$.

Now we complete the proof of Theorem 3.13 by showing the following lemma:

Lemma 3.17. $\left(\alpha_{\gamma}, R_{\gamma}\right)$ and $(\alpha, R)$ are equivalent.

Proof. By replacing $(\alpha, R)$ with a suitable equivalent one, we can assume that $u<v$ implies that $a<b$ for any $(a, u) \in R_{0}(u)$ and $(b, v) \in R_{0}(v)$ such that $0 \leq u, v \leq t-1$. Then the construction of $\gamma$ ensures that $\left(\alpha_{\gamma}, R_{\gamma}\right)=(\alpha, R)$.

3.4. Consequences. For any complete mutation loop $\gamma$, we denote by $F(\gamma)$ the pair $\left(\alpha_{\gamma}, R_{\gamma}\right)$ defined in Section 3.2. For any pair $(\alpha, R)$ of a T-datum and a consistent subset $R$ for $\alpha$, we denote $G(\alpha, R)$ by the complete mutation loop defined in Section 3.3.

We define

$$
\mathrm{Ml}_{r}=\{[\gamma] \mid \gamma \text { is a complete mutation loop of length } r\}
$$

where $[\gamma]$ is the equivalence class of $\gamma$ (see Definition 2.9). We also define

$$
\operatorname{Td}_{r}=\{\alpha \mid \alpha \text { is a T-datum of size } r\}
$$


and

$$
\operatorname{Td}_{r}^{\prime}=\left\{[(\alpha, R)] \mid \alpha \in \operatorname{Td}_{r} \text { and } R \subseteq[1, r] \times \mathbb{Z} \text { is consistent for } \alpha\right\},
$$

where $[(\alpha, R)]$ is the equivalence class of $(\alpha, R)$ (see Definition 3.4). We define

$$
\hat{F}_{r}: \mathrm{Ml}_{r} \rightarrow \mathrm{Td}_{r}^{\prime}, \quad \hat{G}_{r}: \mathrm{Td}_{r}^{\prime} \rightarrow \mathrm{Ml}_{r}
$$

by $\hat{F}_{r}([\gamma])=[F(\gamma)]$ and $\hat{G}_{r}([(\alpha, R)])=[G(\alpha, R)]$.

Theorem 3.18. $\hat{F}_{r} \circ \hat{G}_{r}=$ id and $\hat{G}_{r} \circ \hat{F}_{r}=$ id.

Proof. We first show that $\hat{F}_{r}$ and $\hat{G}_{r}$ are well-defined. Let $\gamma$ and $\gamma^{\prime}$ be equivalent complete mutation loops, and $\rho$ be the permutation in Definition 2.9. Then we have $A_{\gamma^{\prime}, \pm}=\rho\left(A_{\gamma, \pm}\right)$ and $R_{\gamma^{\prime}}=\rho\left(R_{\gamma}\right)$. Thus $\hat{F}_{r}$ is well-defined.

Let $(\alpha, R)$ and $\left(\alpha^{\prime}, R^{\prime}\right)$ be equivalent pairs of T-data and consistent subsets for them. Let $\rho \in \mathfrak{S}_{r}$ be the permutation in Definition 3.4. Let $\gamma=(B, d, \mathbf{i}, \nu)$ and $\gamma^{\prime}=\left(B^{\prime}, d^{\prime}, \mathbf{i}^{\prime}, \nu^{\prime}\right)$ be the mutation loops given by $\gamma=G(\alpha, R)$ and $\gamma^{\prime}=G\left(\alpha^{\prime}, R^{\prime}\right)$, respectively. Then the bijection $f_{\rho, u}: R(u) \rightarrow R^{\prime}(u)$ defined by $f_{\rho, u}(a, u)=(\rho(a), u)$ satisfies $B^{\prime}=f_{\rho, 0}(B), d^{\prime}=f_{\rho, 0}(d)$ and $\nu^{\prime}=f_{\rho, 0} \circ \nu \circ f_{\rho, 0}^{-1}$. Moreover, $R_{0}^{\prime}(u)$ and $f_{\rho, u}\left(R_{0}(u)\right)$ coincide as sets. Thus $\gamma$ and $\gamma^{\prime}$ are equivalent, and $\hat{G}_{r}$ is well-defined.

By Lemma 3.17, we have $\hat{F}_{r} \circ \hat{G}_{r}=$ id. It remains to show that $\hat{G} \circ \hat{F}=$ id. Let $\gamma$ be any complete mutation loop, and $I$ be the index set of $\gamma$. Let $\gamma^{\prime}=G\left(\alpha_{\gamma}, R_{\gamma}\right)$. Then the index set $I^{\prime}$ of $\gamma^{\prime}$ is given by $I^{\prime}=\left\{(a, u) \in R_{\gamma} \mid 0 \leq u<p_{a}\right\}$. Let $f: I \rightarrow I^{\prime}$ be the map defined by

$$
f(i)= \begin{cases}(\pi(i, 0), 0) & \text { if }(i, 0) \in P_{\gamma}, \\ (\pi(s(i, 0)), \lambda(i, 0)) & \text { if }(i, 0) \notin P_{\gamma},\end{cases}
$$

where $\pi, s$, and $\lambda, P_{\gamma}$ are defined in Section 2.2. It is easy to check that $f$ is a bijection, and gives the equivalence between $\gamma$ and $\gamma^{\prime}$.

For any consistent subset $R \subseteq[1, r] \times \mathbb{Z}$ for a T-datum of size $r$, we define the set $R_{\text {in }}$ (the subscript "in" stands for initial) by $R_{\text {in }}=\left\{(a, p) \in R \mid 0 \leq p<p_{a}\right\}$.

Theorem 3.19. Let $\alpha$ be a T-datum of size $r$. Suppose that $R \subseteq[1, r] \times \mathbb{Z}$ is consistent for $\alpha$. Let $\mathbb{P}$ be a semifield, and $\mathcal{F}$ be a filed that is isomorphic to the field of rational functions over $\mathbb{Q P}$ in $\left|R_{\text {in }}\right|$ variables. Let $x=\left(x_{a, p}\right)_{(a, p) \in R_{\mathrm{in}}}$ be an $R_{\mathrm{in}}$-tuple of elements in $\mathcal{F}$ forming a free generating set. Let $Y=\left(Y_{a}(u)\right)_{(a, u) \in R}$ be a solution of the $Y$-system associated with $(\alpha, R)$ in $\mathbb{P}$. Then there exists a unique $R_{\text {in }}$-labeled $Y$-seed $(B, y)$ in $\mathbb{P}$ such that

(1) there exists a unique injective $\mathbb{Z} \mathbb{P}$-algebra homomorphism $\iota: \mathcal{T}^{\circ}(\alpha, R, Y) \hookrightarrow \mathcal{A}(B, y, x)$ such that $\iota\left(T_{a}(p)\right)=x_{a, p}$ for any $(a, p) \in R_{\mathrm{in}}$,

(2) $\iota\left(T_{a}(u)\right)$ is a cluster variable in $\mathcal{A}(B, y, x)$ for any $(a, u) \in R$,

(3) the image of the relation (3.5) by $\iota$ is an exchange relation in $\mathcal{A}(B, y, x)$ for any $(a, u) \in R$.

Proof. Let $\gamma=(B, d, \mathbf{i}, \nu)$ be the mutation loop given by $\gamma=G(\alpha, R)$. By Lemma 3.17, we can assume that $\alpha=\alpha_{\gamma}$ and $R=R_{\gamma}$. We define a family of elements $y=\left(y_{a, p}\right)_{(a, p) \in R_{\text {in }}}$ in $\mathbb{P}$ by

$$
y_{a, p}=Y_{a}(p) \frac{\prod_{b=1}^{r} \prod_{q=0}^{p}\left(1 \oplus Y_{b}(p-q)^{-1}\right)^{\check{n}_{a b ; q}^{+}}}{\prod_{b=1}^{r} \prod_{q=0}^{p}\left(1 \oplus Y_{b}(p-q)\right)^{\check{n}_{a b ; q}^{-}}} .
$$

Then we can define a family of seeds $(B(u), y(u), x(u))_{u \in \mathbb{Z}}$ by $(2.14)$, where $(B(0), y(0), x(0))=(B, y, x)$. Note that $\mathbf{i}(u)=\left(\vec{\varphi}_{u}\right)^{-1}\left(R_{0}(u)\right)$ for any $u \in \mathbb{Z}$ by the commutativity of $\psi^{t}$ and $\varphi$, where we define $\vec{\varphi}_{u}: R(0) \rightarrow R(u)$ by

$$
\vec{\varphi}_{u}= \begin{cases}\varphi_{u-1} \circ \cdots \circ \varphi_{0} & \text { if } u \geq 0 \\ \left(\varphi_{-1} \circ \cdots \circ \varphi_{u}\right)^{-1} & \text { if } u<0\end{cases}
$$


We define $Y^{\prime}=\left(Y_{a}^{\prime}(u)\right)_{(a, u) \in R_{\gamma}}$ by $(2.10)$, where $Y_{a}(u)$ in $(2.10)$ is replaced with $Y_{a}^{\prime}(u)$. Then we have $Y_{a}(p)=Y_{a}^{\prime}(p)$ for any $(a, p) \in R_{\text {in }}$ by (3.11). Moreover, $Y$ and $Y^{\prime}$ are solutions of the same Y-system, we have $Y_{a}(u)=Y_{a}^{\prime}(u)$ for any $(a, u) \in R$. We define $T_{a}^{\prime}(u)$ for any $(a, u) \in R_{\gamma}$ by (2.15), where $T_{a}(u)$ in (2.15) is replaced with $T_{a}^{\prime}(u)$. Let $\mathcal{A}_{\gamma}(B, y, x)$ be the $\mathbb{Z} \mathbb{P}$-subalgebra of $\mathcal{A}(B, y, x)$ generated by $\left(x_{a, p}(u)\right)_{(a, p) \in R_{\text {in }}, u \in \mathbb{Z}}$. It is also generated by $\left(T_{a}^{\prime}(u)\right)_{(a, p) \in R_{\gamma}}$. We now show that $\mathcal{A}_{\gamma}(B, y, x)$ is isomorphic to $\mathcal{T}^{\circ}(\alpha, R, Y)$. Let $\bar{\iota}: \mathcal{T}^{\circ}(\alpha, R, Y) \rightarrow \mathcal{A}_{\gamma}(B, y, x)$ be the algebra homomorphism defined by $\bar{\iota}\left(T_{a}(u)\right)=$ $T_{a}^{\prime}(u)$. This is well-defined by Proposition 2.12. To construct the inverse of $\bar{\iota}$, we define an algebra homomorphism $\kappa: \mathbb{Z P}\left[x_{a, p}^{ \pm 1}\right]_{(a, p) \in R_{\text {in }}} \rightarrow \mathcal{T}(\alpha, R, Y)$ by $\kappa\left(x_{a, p}^{ \pm 1}\right)=T_{a}(p)^{ \pm 1}$. By the Laurent phenomenon of cluster algebras $[11], \mathcal{A}_{\gamma}(B, y, x)$ is a subalgebra of $\mathbb{Z} \mathbb{P}\left[x_{a, p}^{ \pm 1}\right]_{(a, p) \in R_{\mathrm{in}}}$. Then we have $\kappa\left(T_{a}^{\prime}(u)\right)=T_{a}(u)$ since $\left(T_{a}^{\prime}(u)\right)_{(a, p) \in R_{\gamma}}$ and $\left(T_{a}(u)\right)_{(a, p) \in R}$ satisfy the same T-system. Thus we obtain the algebra homomorphism $\bar{\kappa}: \mathcal{A}_{\gamma}(B, y, x) \rightarrow \mathcal{T}^{\circ}(\alpha, R, Y)$ as the restriction of $\kappa$, which is the inverse of $\bar{\iota}$. Therefore, the existence of a Y-seed satisfying (1)-(3) is proved.

The uniqueness follows from the following facts, which hold for any skew-symmetrizable cluster algebra: (i) given a cluster as a set, the exchange relations involving its elements are uniquely determined [4, Proposition 6.1], (ii) any two clusters that have $|I|-1$ common cluster variables are related by an exchange relation [21, Theorem 5].

Example 3.20 (Somos-4 recurrence). Let $\alpha, R$, and $Y$ be as in Example 3.7. Then the Y-seed given by Theorem 3.19 is

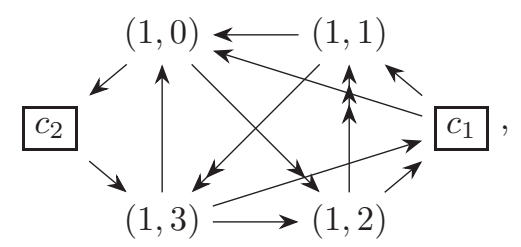

where we represent the $\mathrm{Y}$-seed using a quiver with frozen vertices as in the cluster algebra literature (see e.g., [10]). For instance, $c_{2} \leftarrow i \leftarrow c_{1}$ means $y_{i}=c_{1} c_{2}^{-1}$. The mutation loop $\gamma=(B, d, \mathbf{i}, \nu)=G(\alpha, R)$ is given by

- $B=B(Q)$,

- $d_{i}=1$ for any $i \in I$,

- $\mathbf{i}=\mathbf{i}(0)$ with $\mathbf{i}(0)=((1,0))$,

- $\nu=((1,0)(1,1)(1,2)(1,3))$,

where $Q$ is an underlying quiver in (3.13), and $\nu$ is the cyclic permutation corresponding to the right $\pi / 2$ rotation of the quiver. In fact, the quiver mutation $\mu_{(1,0)}$ is given by

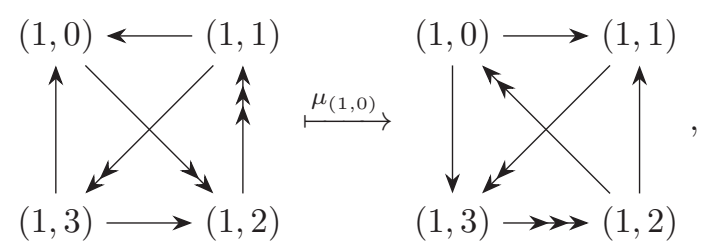

and we have $\mu_{(1,0)}(Q)=\nu(Q)$.

Example 3.21 (Bipartite belt). Let $\alpha$ and $R$ be as in Example 3.8. The mutation loop $\gamma=(B, d, \mathbf{i}, \nu)=$ $G(\alpha, R)$ is given by

- $B=\left(B_{(a, p)(b, q)}\right)_{(a, p),(b, q) \in I}$, where $B_{(a, p)(b, q)}=\epsilon(a) n_{a b}$,

- $d=\left(d_{a, p}\right)_{(a, p) \in I}$, where $D=\operatorname{diag}\left(d_{1}, \ldots, d_{r}\right)$ and $d_{a, p}=d_{a}$,

- $\mathbf{i}=\mathbf{i}(0) \mid \mathbf{i}(1)$ with $\mathbf{i}(0)=\{(a, 0) \mid \epsilon(a)=-1\}$ and $\mathbf{i}(1)=\{(a, 1) \mid \epsilon(a)=1\}$,

- $\nu=\mathrm{id}$, 
where $I=\{(a, 0) \mid \epsilon(a)=-1\} \sqcup\{(a, 1) \mid \epsilon(a)=1\}$. If $A$ is the Cartan matrix of type $A_{2}$, for instance, the quiver $Q(B)$ is given by

$$
Q(B)=(1,0) \longleftarrow(2,1) .
$$

If $A$ is a Cartan matrix of finite type, $\mathcal{A}(B, y, x)$ is a finite type cluster algebra and the embedding $\iota: \mathcal{T}^{\circ}(\alpha, R, Y) \hookrightarrow \mathcal{A}(B, y, x)$ in Theorem 3.19 is an isomorphism [14, Proposition 11.1].

3.5. Tropical T-system. By Theorem 3.19 and the Laurent phenomenon of cluster algebras [11], we obtain the following:

Corollary 3.22. Let $\mathcal{T}^{\circ}(\alpha, R, Y)$ be a T-algebra. Then $T_{a}(u) \in \mathcal{T}^{\circ}(\alpha, R, Y)$ can be written as a Laurent polynomial in $\left(T_{c}(p)\right)_{(c, p) \in R_{\text {in }}}$ with coefficients in $\mathbb{Z P}$, for any $(a, u) \in R$.

Let $R=[1, r] \times \mathbb{Z}$. By Corollary 3.22 , any $T_{a}(u)$ can be uniquely written as

$$
T_{a}(u)=\frac{N}{\prod_{(c, p) \in R_{\mathrm{in}}} T_{c}(p)^{d_{c, p}}},
$$

where $N$ is a polynomial in $\left(T_{c}(p)\right)_{(c, p) \in R_{\text {in }}}$ with coefficients in $\mathbb{Z P}$ which is not divisible by any $T_{c}(p)$ $\left((c, p) \in R_{\text {in }}\right)$. We denote by $\mathfrak{t}_{a}^{(c)}(u)$ the integer $d_{c, 0}$ in (3.14). The family of integers $\left(\mathfrak{t}_{a}^{(c)}(u)\right)_{(a, u) \in[1, r] \times \mathbb{Z}}$ is uniquely determined by the initial conditions

$$
\mathfrak{t}_{a}^{(c)}(p)= \begin{cases}-1 & \text { if }(a, p)=(c, 0) \\ 0 & \text { if }(a, p) \neq(c, 0) \text { and } 0 \leq p<p_{a}\end{cases}
$$

together with the following recurrence relation for each $(a, u) \in[1, r] \times \mathbb{Z}$ :

$$
\sum_{b, p} n_{b a ; p}^{0} \mathfrak{t}_{b}^{(c)}(u+p)=\max \left(\sum_{b, p} n_{b a ; p}^{-} \mathfrak{t}_{b}^{(c)}(u+p), \sum_{b, p} n_{b a ; p}^{+} \mathfrak{t}_{b}^{(c)}(u+p)\right) .
$$

In particular, the integer $\mathfrak{t}_{a}^{(c)}(u)$ is independent of the choice of $Y$. The family of relations (3.16) is called the tropical T-system associated with $\alpha$.

We also define a family of integers $\left(\hat{\mathfrak{y}}_{a}^{(c)}(u)\right)_{(a, u) \in[1, r] \times \mathbb{Z}}$ by

$$
\begin{aligned}
\hat{\mathfrak{y}}_{a}^{(c)}(u) & =\sum_{b, p}\left(n_{b a ; p}^{-} \mathfrak{t}_{b}^{(c)}(u+p)-n_{b a ; p}^{+} \mathfrak{t}_{b}^{(c)}(u+p)\right) \\
& =\sum_{b, p}\left(\left(n_{b a ; p}^{0}-n_{b a ; p}^{+}\right) \mathfrak{t}_{b}^{(c)}(u+p)-\left(n_{b a ; p}^{0}-n_{b a ; p}^{-}\right) \mathfrak{t}_{b}^{(c)}(u+p)\right) .
\end{aligned}
$$

By the relation (3.16), we have

$$
\left[ \pm \hat{\mathfrak{y}}_{a}^{(c)}(u)\right]_{+}=\sum_{b, p}\left(n_{b a ; p}^{0}-n_{b a ; p}^{ \pm}\right) \mathfrak{t}_{b}^{(c)}(u+p) .
$$

The following lemma will be used in Section 5 .

Lemma 3.23. The following equalities hold for any T-datum:

(1) For any $a \in[1, r]$, we have

$$
\mathfrak{t}_{a}^{(c)}\left(p_{a}\right)= \begin{cases}1 & \text { if } a=\sigma(c), \\ 0 & \text { otherwise. }\end{cases}
$$

(2) For any $a \in[1, r]$ and $0 \leq p \leq p_{c}$, we have

$$
\mathfrak{t}_{a}^{(c)}(-p)= \begin{cases}-1 & \text { if }(a, p)=(c, 0) \\ 1 & \text { if }(a, p)=\left(\sigma^{-1}(c), p_{c}\right), \\ 0 & \text { otherwise. }\end{cases}
$$


(3) For any $a \in[1, r]$ and $0 \leq p \leq p_{c}$, we have

$$
\left[ \pm \hat{\mathfrak{y}}_{a}^{(c)}(-p)\right]_{+}=n_{c a ; p}^{ \pm}
$$

Proof. (1) is clear from (3.15) and (3.16). We prove (2) by induction on $p$. The case $p=0$ follows from (3.15). Suppose that $p>0$. Then we have

$$
\begin{aligned}
\mathfrak{t}_{a}^{(c)}(-p) & =-\mathfrak{t}_{\sigma(a)}^{(c)}\left(-p+p_{\sigma(a)}\right)+\max \left(\sum_{b, q} n_{b a ; q}^{-} \mathfrak{t}_{b}^{(c)}(-p+q), \sum_{b, q} n_{b a ; q}^{+} \mathfrak{t}_{b}^{(c)}(-p+q)\right) \\
& =\delta_{\sigma(a) c} \delta_{p p_{c}}+\max \left(-n_{c a ; p}^{+},-n_{c a ; p}^{-}\right) \\
& =\delta_{\sigma(a) c} \delta_{p p_{c}}
\end{aligned}
$$

and (2) is proved. We now prove (3). From (3.19), we have

$$
\begin{aligned}
{\left[ \pm \hat{\mathfrak{y}}_{a}^{(c)}(-p)\right]_{+} } & =\sum_{b, q}\left(n_{b a ; q}^{0}-n_{b a ; q}^{ \pm}\right) \mathfrak{t}_{b}^{(c)}(-p+q) \\
& =\mathfrak{t}_{a}^{(c)}(-p)+\mathfrak{t}_{\sigma(a)}^{(c)}\left(-p+p_{\sigma(a)}\right)-n_{c a ; p}^{ \pm} \mathfrak{t}_{c}^{(c)}(0) \\
& =\mathfrak{t}_{a}^{(c)}(-p)+\mathfrak{t}_{\sigma(a)}^{(c)}\left(-p+p_{\sigma(a)}\right)+n_{c a ; p}^{ \pm} .
\end{aligned}
$$

By (1) and (2) in this lemma, we have

$$
\mathfrak{t}_{a}^{(c)}(-p)= \begin{cases}1 & \text { if }(a, p)=\left(\sigma^{-1}(c), p_{c}\right) \\ -1 & \text { if }(a, p)=(c, 0) \\ 0 & \text { otherwise }\end{cases}
$$

and

$$
\mathfrak{t}_{\sigma(a)}^{(c)}\left(-p+p_{\sigma(a)}\right)= \begin{cases}1 & \text { if }(a, p)=(c, 0) \\ -1 & \text { if }(a, p)=\left(\sigma^{-1}(c), p_{c}\right) \\ 0 & \text { otherwise }\end{cases}
$$

Thus we have $\mathfrak{t}_{a}^{(c)}(-p)+\mathfrak{t}_{\sigma(a)}^{(c)}\left(-p+p_{\sigma(a)}\right)=0$. This completes the proof of $(3)$.

3.6. Indecomposable T-data. If $\left(A_{+}, A_{-}, D\right)$ and $\left(A_{+}^{\prime}, A_{-}^{\prime}, D^{\prime}\right)$ are T-data, the direct sum

$$
\left(\left[\begin{array}{cc}
A_{+} & O \\
O & A_{+}^{\prime}
\end{array}\right],\left[\begin{array}{cc}
A_{-} & O \\
O & A_{-}^{\prime}
\end{array}\right],\left[\begin{array}{cc}
D & O \\
O & D^{\prime}
\end{array}\right]\right)
$$

is also a T-datum. A T-datum $\left(A_{+}, A_{-}, D\right)$ is called decomposable if it can be written as a nontrivial direct sum after reordering the indices of matrices. A T-datum that is not decomposable is called indecomposable.

We say that a skew-symmetrizable matrix $B$ is connected if it cannot be written as a nontrivial direct sum. We also say that a connected skew-symmetrizable matrix $B^{\prime}=\left(B_{i j}^{\prime}\right)_{i, j \in I^{\prime}}$ is a connected component of a skew-symmetrizable matrix $B=\left(B_{i j}\right)_{i, j \in I}$ if $I^{\prime} \subseteq I, B_{i j}^{\prime}=B_{i j}$ for any $i, j \in I^{\prime}$, and $B_{i j}=0$ for any $i \in I^{\prime}$ and $j \in I \backslash I^{\prime}$.

Proposition 3.24. Let $\alpha$ be an indecomposable T-datum. Let $I^{\prime}$ be the index set of a connected component of $B$, where $B$ is the skew-symmetrizable matrix in the mutation loop $G(\alpha,[1, r] \times \mathbb{Z})$. Then the set

$$
R^{\prime}:=\bigcup_{u \in \mathbb{Z}} \vec{\varphi}_{u}\left(I^{\prime}\right)
$$

is consistent for $\alpha$, where $\vec{\varphi}_{u}$ is defined by (3.12). Moreover, the index set of $B^{\prime}$ is $I^{\prime}$, where $B^{\prime}$ is the skew-symmetrizable matrix in the mutation loop $G\left(\alpha, R^{\prime}\right)$. 
Proof. Let $(B, d, \mathbf{i}, \nu)=G(\alpha,[1, r] \times \mathbb{Z})$. The bijection $\nu$ is given by $\nu=\varphi_{0}^{-1} \circ \psi$. Let $I$ be the index set of $B$, that is, $I=\left\{(a, p) \mid a \in[1, r], 0 \leq p<p_{a}\right\}$. We recursively define subsets $I_{k}^{\prime} \subseteq I$ for $k \in \mathbb{Z}_{>0}$ by $I_{0}^{\prime}=I^{\prime}$ and $I_{k}^{\prime}=\left.\nu\right|_{I_{k-1}^{\prime}}\left(I_{k-1}^{\prime}\right)$. Then $\left.B\right|_{I_{k}^{\prime}}$ is a connected component of $B$ since mutations preserve connected components. We now prove (R1) and (R2) in Definition 3.3 for $R^{\prime}$. The proof of (R1) and (R2) are almost the same, we only prove $(\mathrm{R} 1)$. Suppose that $(a, u) \in R^{\prime}$. If $\check{n}_{a b ; p}^{0} \neq 0$, then $(b, u-p) \in R^{\prime}$ by the definition of $R^{\prime}$. Suppose that $\check{n}_{a b ; p}^{+}$or $\check{n}_{a b ; p}^{-} \neq 0$. Then we have $(a, u),(b, u-p) \in \vec{\varphi}_{u-p}(I)$ and $\bar{B}_{(b, u-p)(a, u)}(u-p) \neq 0$, where $\bar{B}(u-p)$ is defined in $(3.7)$. This shows that $(a, p)$ and $(b, 0)$ lie in the same connected component $\left.B\right|_{I_{p-u}^{\prime}}$. Thus we have $(b, u-p) \in R^{\prime}$ since $\vec{\varphi}_{u-p}\left(\nu^{u-p}(b, 0)\right)=(b, u-p)$ and $\nu^{u-p}(b, 0) \in I^{\prime}$.

We next prove (R3). Let $t$ be the smallest positive integer such that $I_{t}^{\prime}=I^{\prime}$. We now show that

$$
I=\bigsqcup_{k=0}^{t-1} I_{k}^{\prime}
$$

The equality $I=\bigcup_{k=0}^{t-1} I_{k}^{\prime}$ follows from the fact that $\alpha$ is indecomposable. Suppose that $I_{k_{1}}^{\prime} \cap I_{k_{2}}^{\prime} \neq \emptyset$ for some $0 \leq k_{1}<k_{1}<t$. Then we have $I_{k_{1}}^{\prime}=I_{k_{2}}^{\prime}$ since $\left.B\right|_{I_{k_{1}}^{\prime}}$ and $\left.B\right|_{I_{k_{2}}^{\prime}}$ are connected components of $B$. But this implies $I^{\prime}=I_{k_{2}-k_{1}}^{\prime}$, which contradicts the minimality of $t$. Thus (3.20) is proved. We now have

$$
\begin{aligned}
& \bigcup_{k=0}^{t-1} \psi^{k}\left(R^{\prime}\right)=\bigcup_{k=0}^{t-1} \bigcup_{u \in \mathbb{Z}}\left(\psi^{k} \circ \vec{\varphi}_{u}\right)\left(I^{\prime}\right)=\bigcup_{k=0}^{t-1} \bigcup_{u \in \mathbb{Z}}\left(\vec{\varphi}_{u+k} \circ \nu^{k}\right)\left(I^{\prime}\right) \\
& =\bigcup_{k=0}^{t-1} \bigcup_{u \in \mathbb{Z}} \vec{\varphi}_{u+k}\left(I_{k}^{\prime}\right)=\bigcup_{k=0}^{t-1} \bigcup_{u \in \mathbb{Z}} \vec{\varphi}_{u}\left(I_{k}^{\prime}\right)=\bigcup_{u \in \mathbb{Z}} \vec{\varphi}_{u}\left(\bigcup_{k=0}^{t-1} I_{k}^{\prime}\right) \\
& =\bigcup_{u \in \mathbb{Z}} \vec{\varphi}_{u}(I)=[1, r] \times \mathbb{Z} .
\end{aligned}
$$

It remains to prove the disjointness. Suppose that there exists a element $(a, u) \in \psi^{k_{1}}\left(R^{\prime}\right) \cap \psi^{k_{2}}\left(R^{\prime}\right)$ for some $0 \leq k_{1}<k_{2}<t$. Then we have $\left(\left(\vec{\varphi}_{u-k_{i}}\right)^{-1} \circ \psi^{-k_{i}}\right)(a, u) \in I^{\prime}$ for $i=1$, 2 . Thus we have $\left(\nu^{k_{i}} \circ\left(\vec{\varphi}_{u-k_{i}}\right)^{-1} \circ \psi^{-k_{i}}\right)(a, u) \in I_{k_{i}}^{\prime}$ for $i=1,2$. On the other hand, we have $\nu^{k_{i}} \circ\left(\vec{\varphi}_{u-k_{i}}\right)^{-1} \circ \psi^{-k_{i}}=\left(\vec{\varphi}_{u}\right)^{-1}$. This implies that $\left(\vec{\varphi}_{u}\right)^{-1}(a, u) \in I_{k_{1}}^{\prime} \cap I_{k_{2}}^{\prime}$, which contradicts (3.20).

Corollary 3.25. Let $\alpha=\left(A_{+}, A_{-}, D\right)$ be an indecomposable T-datum of size $r$. Suppose that there exists $a \in[1, r]$ such that both the a-th columns in $N_{+}$and $N_{-}$are zero vectors. Then both $N_{+}$and $N_{-}$are zero matrices.

Proof. Let $(B, d, \mathbf{i}, \nu)=G(\alpha,[1, r] \times \mathbb{Z})$. By the assumption and (3.9), the set $I^{\prime}=\{(a, 0)\}$ is the index set of a connected component of $B$. Then the set $R^{\prime}$ defined in Proposition 3.24 is consistent for $\alpha$. Let $\left(B^{\prime}, d^{\prime}, \mathbf{i}^{\prime}, \nu^{\prime}\right)=G\left(\alpha, R^{\prime}\right)$. By Proposition 3.24, $B^{\prime}$ is an $I^{\prime} \times I^{\prime}$ matrix. This implies that $B^{\prime}=0$ since any skew-symmetrizable matrix of size 1 should be the zero matrix. From (2.17) and Lemma 3.17, we have $N_{ \pm}=0$.

\section{EXAMPLES OF T-DATA}

\subsection{Period 1 quivers.}

Theorem 4.1. Let $p>0$ be a positive integer, and $a(z)=1+n_{1} z+\cdots+n_{p-1} z^{p-1}+z^{p} \in \mathbb{Z}[z]$ be a monic palindromic polynomial of degree $p$, that is, $n_{q}=n_{p-q}$ for any $0<q<p$. Let $d$ be any positive integer. Then the triple $\alpha=\left(A_{+}, A_{-},[d]\right)$ given by $A_{ \pm}=N_{0}-N_{ \pm}$where

$$
N_{0}=\left[1+z^{p}\right], \quad N_{+}=\left[\sum_{q=1}^{p-1}\left[n_{q}\right]_{+} z^{q}\right], \quad N_{-}=\left[\sum_{q=1}^{p-1}\left[-n_{q}\right]_{+} z^{q}\right]
$$


is a T-datum of size 1. Furthermore, any T-datum of size 1 is of this form.

Proof. The conditions (N1)-(N4) follow immediately from the definition. Since $a(z)$ is a monic palindromic polynomial of degree $p$, both $z^{-p / 2} A_{+}$and $z^{-p / 2} A_{-}$are $\dagger$-invariant. This implies that $A_{+} A_{-}^{\dagger}=A_{-} A_{+}^{\dagger}$. Thus $\alpha$ is a T-datum.

Conversely, let $\alpha=\left(A_{+}, A_{-},[d]\right)=\left(N_{0}-N_{+}, N_{0}-N_{-},[d]\right)$ be any T-datum of size 1 . We now identify $1 \times 1$ matrices with their entries. By the condition (N1), the matrix $N_{0}$ can be written as $N_{0}=1+z^{p}$ for some positive integer $p>0$. Then the matrices $N_{ \pm}$can be written as $N_{\varepsilon}=\sum_{q=1}^{p-1} n_{q}^{\varepsilon} z^{q}$ since the degrees of $N_{ \pm}$are greater that 0 and less than $p$ by the condition (N3). We also have $n_{q}^{\varepsilon} \in \mathbb{Z}_{\geq 0}$ by the condition (N2). By the condition (N4), these numbers can be written as $n_{q}^{ \pm}=\left[ \pm n_{q}\right]_{+}$, where we define $n_{q}:=n_{q}^{+}-n_{q}^{-}$. We now show by induction on $q$ that $n_{q}^{ \pm}=n_{p-q}^{ \pm}$for any $0 \leq q \leq p$, where we set $n_{0}^{ \pm}=n_{p}^{ \pm}=0$. The case $q=0$ is obvious from the definition. Suppose that $q>0$. Let $m_{q}$ be the coefficient of $z^{p-q}$ in the polynomial $N_{0} N_{+}^{\dagger}+N_{+} N_{-}^{\dagger}+N_{-} N_{0}^{\dagger}$, that is,

$$
m_{q}=n_{q}^{+}+n_{p-q}^{-}+\sum_{k=0}^{q} n_{p-q+k}^{+} n_{k}^{-} .
$$

On the other hand, $m_{q}$ is also the coefficient of $z^{p-q}$ in the polynomial $N_{0} N_{-}^{\dagger}+N_{-} N_{+}^{\dagger}+N_{+} N_{0}^{\dagger}$ by the symplectic relation. Thus we obtain

$$
m_{q}=n_{q}^{-}+n_{p-q}^{+}+\sum_{k=0}^{q} n_{p-k}^{-} n_{q-k}^{+} .
$$

The sum parts in (4.1) and (4.2) coincide by the induction hypothesis, so we obtain $n_{q}^{+}+n_{p-q}^{-}=n_{q}^{-}+n_{p-q}^{+}$. Then we conclude from (N4) that $n_{q}^{ \pm}=n_{p-q}^{ \pm}$.

Let $\alpha$ be a T-datum of size 1 given in Theorem 4.1. Let $\gamma=(B, d, \mathbf{i}, \nu)$ be the complete mutation loop given by $\gamma=G(\alpha,\{1\} \times \mathbb{Z})$. Then the index set $I$ of $B$ is given by $I=\{(1, i) \in\{1\} \times \mathbb{Z} \mid 0 \leq i<p\}$. We identify $I$ with the set $\{0,1, \ldots, p-1\}$. Then $\mathbf{i}=\mathbf{i}(0)$ with $\mathbf{i}(0)=(0)$, and $\nu$ is the cyclic permutation given by $\nu(i)=i+1(\bmod t)$. The exchange matrix $B=\left(B_{i j}\right)_{i, j \in I}$ can be computed from the formula (3.9) as follows:

$$
B_{i j}=-n_{i-j}+n_{j-i}+\sum_{k=0}^{\min (i, j)}\left(n_{i-k}^{+} n_{j-k}^{-}-n_{i-k}^{-} n_{j-k}^{+}\right),
$$

where $n_{i}^{ \pm}:=\left[ \pm n_{i}\right]_{+}$and $n_{i}:=0$ unless $0<i<p$.

Remark 4.2. The formula (4.3) is precisely the general solution of period 1 quivers given by Fordy and Marsh [16, Theorem 6.1]. We can regard Theorem 4.1 as another proof of the classification for period 1 quivers, which was also given in [16, Theorem 6.1].

In Example 3.7 and 3.20 (the Somos-4 recurrence), we give an example of a T-datum of size 1 and a period 1 quiver.

4.2. Commuting Cartan matrices. In this section, we give T-data associated with pairs of Cartan matrices, which are generalization of T-data associated with bipartite belts in Example 3.8 and 3.21.

Definition 4.3. A matrix $C=\left(c_{a b}\right)_{a, b \in[1, r]} \in \operatorname{Mat}_{r \times r}(\mathbb{Z})$ is called a symmetrizable weak generalized Cartan matrix if

(1) $c_{a a} \leq 2$ for any $a$,

(2) $c_{a b} \geq 0$ for any $a, b$,

(3) there exists a positive integer diagonal matrix $D$ such that $C D$ is a symmetric matrix. 
The diagonal matrix $D$ is called a (right) symmetrizer of $C$. Note that a symmetrizable generalized Cartan matrix is a symmetrizable weak generalized Cartan matrix satisfying $c_{a a}=2$ for any $a \in[1, r]$.

Proposition 4.4. Let $A$ and $A^{\prime}$ be symmetrizable weak generalized Cartan matrices that have a common symmetrizer $D$. Let $N=\left(n_{a b}\right)_{a, b \in[1, r]}:=2 I_{r}-A$ and $N^{\prime}=\left(n_{a b}^{\prime}\right)_{a, b \in[1, r]}:=2 I_{r}-A^{\prime}$. Then the triple $\alpha=\left(A_{+}, A_{-}, D\right)$ defined by

$$
N_{0}=\left(1+z^{2}\right) I_{r}, \quad N_{+}=z N, \quad N_{-}=z N^{\prime}
$$

is a T-datum if and only if $A A^{\prime}=A^{\prime} A$ and $n_{a b} n_{a b}^{\prime}=0$ for any $a, b \in[1, r]$.

Proof. The conditions (N1)-(N3) are obvious by the definition. We also have $N_{0} D=D N_{0}$ and $D^{-1} N_{ \pm} D \in$ $\operatorname{Mat}_{r \times r}(\mathbb{Z}[z])$ by the definition. The condition (N4) is equivalent to $n_{a b} n_{a b}^{\prime}=0$ for any $a, b \in[1, r]$. Thus it is sufficient to show that the symplectic relation is equivalent to $A A^{\prime}=A^{\prime} A$. Clearly, $A A^{\prime}=A^{\prime} A$ if and only if $N N^{\prime}=N^{\prime} N$. We now have

$$
\begin{aligned}
A_{+} & D A_{-}^{\dagger}-A_{-} D A_{+}^{\dagger} \\
= & \left(\left(1+z^{2}\right) I_{r}-z N\right) D\left(\left(1+z^{-2}\right) I_{r}-z^{-1} N^{\prime \top}\right) \\
& -\left(\left(1+z^{2}\right) I_{r}-z N^{\prime}\right) D\left(\left(1+z^{-2}\right) I_{r}-z^{-1} N^{\top}\right) \\
= & \left(z+z^{-1}\right)\left(-N D-D N^{\prime \top}+D N^{\top}+N^{\prime \top} D\right)+N D N^{\prime \top}-N^{\prime} D N^{\top} \\
= & N D N^{\prime \top}-N^{\prime} D N^{\top} \\
= & \left(N N^{\prime}-N^{\prime} N\right) D
\end{aligned}
$$

which completes the proof.

Proposition 4.5. Suppose that $\alpha$ given in Proposition 4.4 is a T-datum. Suppose further that there exists a function $\epsilon:[1, r] \rightarrow\{1,-1\}$ such that $n_{a b}$ or $n_{a b}^{\prime}>0$ implies $\epsilon(a) \neq \epsilon(b)$ for any $a, b \in[1, r]$. Then the set

$$
R_{\epsilon}:=\left\{(a, u) \in[1, r] \times \mathbb{Z} \mid \epsilon(a)=(-1)^{u-1}\right\}
$$

is consistent for $\alpha$.

Proof. The conditions (R1) and (R2) follow from the assumption on the function $\epsilon$. The condition (R3) is satisfied by setting $t=2$.

Let $\gamma=(B, d, \mathbf{i}, \nu)=G\left(\alpha, R_{\epsilon}\right)$ be the mutation loop obtained from the data given in Proposition 4.5. The index set of $B$ is given by

$$
I=\{(a, 0) \mid \epsilon(a)=-1\} \sqcup\{(a, 1) \mid \epsilon(a)=1\},
$$

and we identify it with $[1, r]$ by taking the first components. Then $B=\left(B_{a b}\right)_{a, b \in I}$ is given by

$$
B_{a b}= \begin{cases}-\epsilon(a) n_{a b} & \text { if } n_{a b}^{\prime}=0 \\ +\epsilon(a) n_{a b}^{\prime} & \text { if } n_{a b}=0\end{cases}
$$

The symmetrizer $d$ is given by $d=\left(d_{a}\right)_{a \in I}$, where $d_{a}$ is the $a$-th entry in the common symmetrizer $D$. The sequence $\mathbf{i}$ is given by $\mathbf{i}=\mathbf{i}(0) \mid \mathbf{i}(1)$ with $\mathbf{i}(0)=\{a \in I \mid \epsilon(a)=-1\}$ and $\mathbf{i}(1)=\{a \in I \mid \epsilon(a)=1\}$. The permutation $\nu$ is the trivial permutation.

Remark 4.6. If $B$ is skew-symmetric, the corresponding quiver $Q(B)$ is called a bipartite recurrent quiver [19]. Galashin and Pylyavskyy developed the classification theory of bipartite recurrent quivers [19, 17, 18]. In particular, they gave a complete classification of bipartite recurrent quivers with which the associated T-system is periodic. 
Example 4.7 (Tensor product construction). Let $\bar{A}$ and $\bar{A}^{\prime}$ be symmetrizable weak generalized Cartan matrices of size $\bar{r}$ and $\bar{r}^{\prime}$, respectively. Suppose that one of them is non-weak. Let $D$ and $D^{\prime}$ be right symmetrizers of $\bar{A}$ and $\bar{A}^{\prime}$, respectively. Let $A=\bar{A} \otimes I_{\bar{r}^{\prime}}, A^{\prime}=I_{\bar{r}} \otimes \bar{A}^{\prime}$, and $D=\bar{D} \otimes \bar{D}^{\prime}$. The matrices $A$ and $A^{\prime}$ are symmetrizable weak generalized Cartan matrices that have the common symmetrizer $D$. Then the triple $\alpha=\left(A_{+}, A_{-}, D\right)$ given in Proposition 4.4 is a T-datum of size $\bar{r} \bar{r}^{\prime}$ since $\left(\bar{A} \otimes I_{\bar{r}^{\prime}}\right)\left(I_{\bar{r}} \otimes \bar{A}^{\prime}\right)=$ $\left(I_{\bar{r}} \otimes \bar{A}^{\prime}\right)\left(\bar{A} \otimes I_{\bar{r}^{\prime}}\right)$ and $n_{a b} \delta_{a^{\prime} b^{\prime}} \delta_{a b} n_{a^{\prime} b^{\prime}}^{\prime}=0$ for any $a, b \in[1, \bar{r}]$ and $a^{\prime}, b^{\prime} \in\left[1, \bar{r}^{\prime}\right]$.

Suppose further that both $\bar{A}$ and $\bar{A}^{\prime}$ are bipartite by functions $\bar{\epsilon}$ and $\bar{\epsilon}$, respectively. Then the function $\epsilon:[1, \bar{r}] \times\left[1, \bar{r}^{\prime}\right] \rightarrow\{1,-1\}$ defined by $\epsilon\left(a, a^{\prime}\right)=\bar{\epsilon}(a) \bar{\epsilon}^{\prime}\left(a^{\prime}\right)$ satisfies the assumption in Proposition 4.5. Thus we get the consistent subset $R_{\epsilon}$ for $\alpha$. For example, let

$$
A=\left[\begin{array}{ccc}
2 & -1 & 0 \\
-1 & 2 & -1 \\
0 & -1 & 2
\end{array}\right], \quad A^{\prime}=\left[\begin{array}{cc}
2 & -1 \\
-1 & 2
\end{array}\right]
$$

be Cartan matrices of types $A_{3}$ and $A_{2}$, respectively. Define $\bar{\epsilon}$ and $\bar{\epsilon}^{\prime}$ by $\bar{\epsilon}(1)=\bar{\epsilon}(3)=\bar{\epsilon}^{\prime}(2)=1$ and $\bar{\epsilon}(2)=\bar{\epsilon}^{\prime}(1)=-1$. Then the bipartite recurrent quiver $Q(B)$ is given by

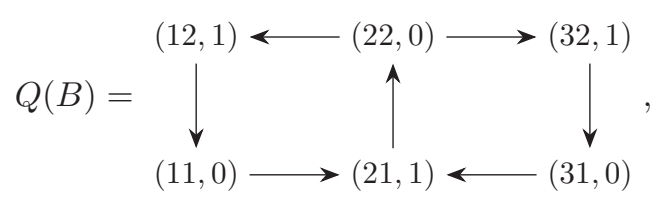

where we denote $\left(a, a^{\prime}\right)$ by $a a^{\prime}$.

Example 4.8 (Tadpole type). Although Proposition 4.5 is for bipartite Cartan matrices, non-bipartite Cartan matrices are sometimes interesting. Let $A=2 I_{r}$ and $A^{\prime}=\left(2 \delta_{a b}-n_{a b}^{\prime}\right)_{a, b \in[1, r]}$ where

$$
n_{a b}^{\prime}= \begin{cases}1 & \text { if }|a-b|=1 \\ 1 & \text { if } a=b=r \\ 0 & \text { otherwise }\end{cases}
$$

The matrix $A^{\prime}$ is called the Cartan matrix of the tadpole type $T_{r}$. The tadpole type is non-bipartite since (4.5) has a non-zero entry in the diagonal. Let $D=I_{r}$. Then $\alpha$ in Proposition 4.4 is a T-datum. For example, $\alpha=\left(A_{+}, A_{-}, I_{r}\right)$ for $r=3$ is given by

$$
A_{+}=\left[\begin{array}{ccc}
1+z^{2} & 0 & 0 \\
0 & 1+z^{2} & 0 \\
0 & 0 & 1+z^{2}
\end{array}\right], \quad A_{-}=\left[\begin{array}{ccc}
1+z^{2} & -z & 0 \\
-z & 1+z^{2} & -z \\
0 & -z & 1-z+z^{2}
\end{array}\right] .
$$

Let $\gamma=(B, d, \mathbf{i}, \nu)$ be the mutation loop given by $\gamma=G(\alpha,[1, r] \times \mathbb{Z})$. Then the quiver mutation $Q(B) \stackrel{\mu_{\mathbf{i}}}{\longmapsto} \mu_{\mathbf{i}}(Q(B))=\nu(Q(B))$ is given as follows (we set $r=3$ for simplicity) :

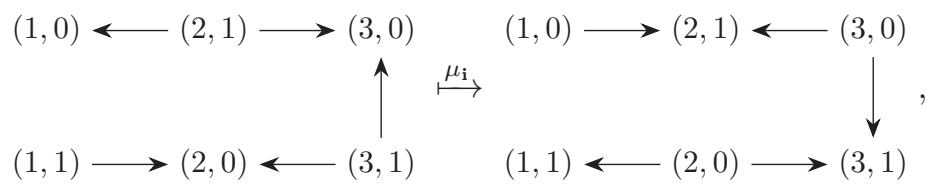

where $\mathbf{i}=\mathbf{i}(0)=\{(a, 0) \mid a \in[1, r]\}$, and $\nu(a, p)=(a, 1-p)$ is the permutation that swaps vertices at the same position in the top and bottom rows. Intuitively, this mutation loop is explained as follows. If we identify the vertices lying in the same $\nu$-orbits and forget the orientation of the quiver, we obtain the following graph:

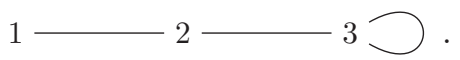


This is the Dynkin diagram of type $T_{r}$. Therefore, the mutation loop involves the "folding method" that constructs $T_{r}$ diagram from $A_{2 r}$ diagram. In general, one advantage of the strategy of constructing mutation loops from T-data is that it can "automatically" perform such a folding method.

4.3. T-systems associated with quantum affinizations. In this section, we assign T-data to generalized Cartan matrices that satisfy a certain condition, including all finite and affine types. The T-data in this section are different from that in Section 4.2 even though both use Cartan matrices.

Fix a positive integer $n$. Let $C=\left(c_{a b}\right)_{1 \leq a, b \leq n}$ be a symmetrizable generalized Cartan matrix. We assume that $C$ is indecomposable. Let $\operatorname{diag}\left(c_{1}, \ldots, c_{n}\right)$ be a left symmetrizer of $C$. We define integers $t_{a}$ $(1 \leq a \leq n)$ by

$$
t_{a}=c_{a}^{-1} \operatorname{lcm}\left(c_{1}, \ldots, c_{n}\right)
$$

We also define integers $t_{a b}(1 \leq a, b \leq n)$ by

$$
t_{a b}=c_{a}^{-1} \operatorname{lcm}\left(c_{a}, c_{b}\right)
$$

These integers do not depend on the choice of a symmetrizer. Let $[k]_{z} \in \mathbb{Z}\left[z^{ \pm 1}\right]$ be the $z$-integer defined by

$$
\begin{aligned}
{[k]_{z} } & =\frac{z^{k}-z^{-k}}{z-z^{-1}} \\
& =z^{k-1}+z^{k-3}+\cdots+z^{-(k-3)}+z^{-(k-1)} .
\end{aligned}
$$

We denote $[k]_{z^{c_{a}}}$ by $[k]_{z_{a}}$.

Let $\ell$ be an integer with $\ell \geq 2$. Let $H$ be the index set defined by

$$
H=\left\{(a, m) \mid 1 \leq a \leq n, 1 \leq m \leq t_{a} \ell-1\right\} .
$$

We often denote an element $(a, m) \in H$ by $a m$. For any $(a, m),(b, k) \in H$, we define polynomials $\tilde{n}_{a m, b k}^{0}, \tilde{n}_{a m, b k}^{+}, \tilde{n}_{a m, b k}^{-} \in \mathbb{Z}\left[z^{ \pm 1}\right]$ by

$$
\begin{aligned}
& \tilde{n}_{a m, b k}^{0}=[2]_{z_{a}} \delta_{a b}, \\
& \tilde{n}_{a m, b k}^{+}=\delta_{a b}\left(\delta_{m, k+1}+\delta_{m, k-1}\right), \\
& \tilde{n}_{a m, b k}^{-}= \begin{cases}t_{a b}^{-1}\left|c_{a b}\right|\left[t_{b a}-|p-k|\right]_{z_{b}} & \text { if } a \sim b, p \in \mathbb{Z}, \text { and }|p-k|<t_{b a}, \\
0 & \text { otherwise, }\end{cases}
\end{aligned}
$$

where we write $a \sim b$ if $c_{a b}<0$, and $p=m t_{a b}^{-1} t_{b a}$. We define two $H \times H$-matrices $\widetilde{A}_{+}$and $\widetilde{A}_{-}$by

$$
\widetilde{A}_{ \pm}=\left(\tilde{n}_{a m, b k}^{0}-\tilde{n}_{a m, b k}^{ \pm}\right)_{a m, b k \in H}
$$

To illustrate the pair of matrices $\left(\widetilde{A}_{+}, \widetilde{A}_{-}\right)$, it is useful to consider the graph $\Gamma\left(\widetilde{A}_{+}, \widetilde{A}_{-}\right)$defined as follows:

- the set of vertices of $\Gamma\left(\widetilde{A}_{+}, \widetilde{A}_{-}\right)$is $H$,

- for any pair of vertices $(a, m),(b, k) \in H$, we draw a blue edge equipped with the pair of polynomials $\left(f_{+}, g_{+}\right):=\left(\tilde{n}_{a m, b k}^{+}, \tilde{n}_{b k, a m}^{+}\right)$, and a red edge equipped with the pair of polynomials $\left(f_{-}, g_{-}\right):=$ $\left(\tilde{n}_{a m, b k}^{-}, \tilde{n}_{b k, a m}^{-}\right)$:

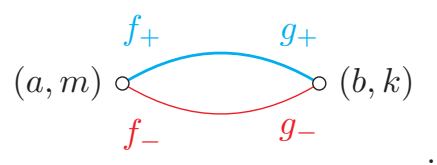


For a red edge

$$
(a, m) \circ \mathrm{f}_{-} g_{-} \circ(b, k)
$$

we use the following abbreviations:

$$
\begin{array}{ll}
\circ & \text { if }\left(f_{-}, g_{-}\right)=(0,0), \\
\circ & \text { if }\left(f_{-}, g_{-}\right)=(1,1), \\
\circ & \text { if }\left(f_{-}, g_{-}\right)=\left(1,[2]_{z_{a}}\right), \\
& \text { if }\left(f_{-}, g_{-}\right)=\left(1,[3]_{z_{a}}\right), \\
& \text { if }\left(f_{-}, g_{-}\right)=(0,1), \\
& \text { if }\left(f_{-}, g_{-}\right)=\left(0,[2]_{z_{a}}\right), \\
& \text { if }\left(f_{-}, g_{-}\right)=\left(0,[3]_{z_{a}}\right) .
\end{array}
$$

We may use the same abbreviations for blue edges, but these are not needed here. When we use these abbreviations, we keep in mind the symmetrizer.

Example 4.9. Consider a Cartan matrix of type $F_{4}$ :

$$
C=\left[\begin{array}{rrrr}
2 & -1 & 0 & 0 \\
-1 & 2 & -1 & 0 \\
0 & -2 & 2 & -1 \\
0 & 0 & -1 & 2
\end{array}\right]
$$

and choose a symmetrizer as $\operatorname{diag}(2,2,1,1)$. When $\ell=2$, the index set $H$ is given by

$$
H=\{(1,1),(2,1),(3,1),(3,2),(3,3),(4,1),(4,2),(4,3)\}
$$

and the matrices $\widetilde{A}_{+}$and $\widetilde{A}_{-}$are given by

$$
\begin{aligned}
& \widetilde{A}_{+}=\left[\begin{array}{cccccccc}
{[2]_{z^{2}}} & 0 & 0 & 0 & 0 & 0 & 0 & 0 \\
0 & {[2]_{z^{2}}} & 0 & 0 & 0 & 0 & 0 & 0 \\
0 & 0 & {[2]_{z}} & -1 & 0 & 0 & 0 & 0 \\
0 & 0 & -1 & {[2]_{z}} & -1 & 0 & 0 & 0 \\
0 & 0 & 0 & -1 & {[2]_{z}} & 0 & 0 & 0 \\
0 & 0 & 0 & 0 & 0 & {[2]_{z}} & -1 & 0 \\
0 & 0 & 0 & 0 & 0 & -1 & {[2]_{z}} & -1 \\
0 & 0 & 0 & 0 & 0 & 0 & -1 & {[2]_{z}}
\end{array}\right] \\
& \widetilde{A}_{-}=\left[\begin{array}{cccccccc}
{[2]_{z^{2}}} & -1 & 0 & 0 & 0 & 0 & 0 & 0 \\
-1 & {[2]_{z^{2}}} & -1 & -[2]_{z} & -1 & 0 & 0 & 0 \\
0 & 0 & {[2]_{z}} & 0 & 0 & -1 & 0 & 0 \\
0 & -1 & 0 & {[2]_{z}} & 0 & 0 & -1 & 0 \\
0 & 0 & 0 & 0 & {[2]_{z}} & 0 & 0 & -1 \\
0 & 0 & -1 & 0 & 0 & {[2]_{z}} & 0 & 0 \\
0 & 0 & 0 & -1 & 0 & 0 & {[2]_{z}} & 0 \\
0 & 0 & 0 & 0 & -1 & 0 & 0 & {[2]_{z}}
\end{array}\right] .
\end{aligned}
$$



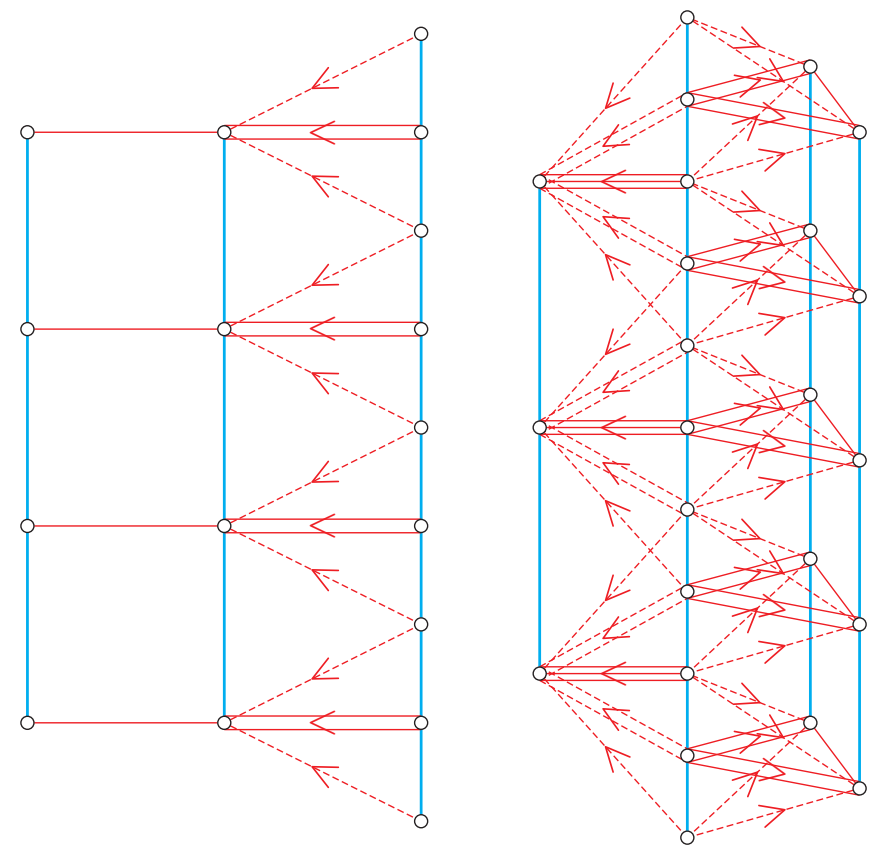

Figure 3. Examples of the diagram $\Gamma\left(\widetilde{A}_{+}, \widetilde{A}_{-}\right)$.

The diagram $\Gamma\left(\widetilde{A}_{+}, \widetilde{A}_{-}\right)$is given by

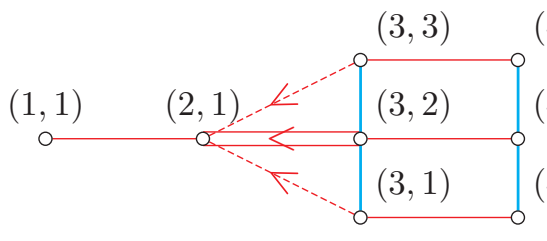

More complicated examples are given in Figure 3. The left diagram is of

$$
C=\left[\begin{array}{rrr}
2 & -1 & 0 \\
-1 & 2 & -1 \\
0 & -2 & 2
\end{array}\right]
$$

(the Cartan matrix of type $B_{3}$ ) and $\ell=5$, and the right diagram is of

$$
C=\left[\begin{array}{rrrr}
2 & -1 & 0 & 0 \\
-3 & 2 & -2 & -2 \\
0 & -1 & 2 & -1 \\
0 & -1 & -1 & 2
\end{array}\right]
$$

and $\ell=2$.

Proposition 4.10. The red part of the diagram $\Gamma\left(\widetilde{A}_{+}, \widetilde{A}_{-}\right)$contains the Dynkin diagram of the transpose of C. More precisely, we have

$$
\left(\left.\widetilde{A}_{-}\right|_{z=1}\right)_{a m, b k}=c_{b a}
$$

if $t_{b a} m=t_{a b} k$. 
Proof. Suppose that $(a, m),(b, k) \in H$ satisfy $t_{b a} m=t_{a b} k$. Note that such pairs exist for any $a, b$ since $m:=t_{a b} \leq t_{a} \leq t_{a} \ell-1$ and $k:=t_{b a} \leq t_{b} \leq t_{b} \ell-1$ satisfy the condition. If $a=b$, we have

$$
\left(\left.\widetilde{A}_{-}\right|_{z=1}\right)_{a m, b k}=\left.\left(\tilde{n}_{a m, a m}^{0}\right)\right|_{z=1}=2,
$$

and this is equal to $c_{a a}$. If $a \neq b$, we have

$$
\left(\left.\widetilde{A}_{-}\right|_{z=1}\right)_{a m, b k}=-\left.\left(\tilde{n}_{a m, b k}^{-}\right)\right|_{z=1}=t_{a b}^{-1} c_{a b} t_{b a}=c_{a} c_{a b} c_{b}^{-1}=c_{b a} .
$$

Lemma 4.11. The matrix $\widetilde{A}_{+} \widetilde{A}_{-}^{\top}$ is symmetric.

Proof. The matrix $\widetilde{A}_{+} \widetilde{A}_{-}^{\top}$ is symmetric if and only if

$$
\begin{aligned}
\sum_{(c, l) \in H}( & \tilde{n}_{a m, c l}^{0} \tilde{n}_{b k, c l}^{+}+\tilde{n}_{a m, c l}^{+} \tilde{n}_{b k, c l}^{-}+\tilde{n}_{a m, c l}^{-} \tilde{n}_{b k, c l}^{0} \\
& \left.\quad-\tilde{n}_{a m, c l}^{0} \tilde{n}_{b k, c l}^{-}-\tilde{n}_{a m, c l}^{-} \tilde{n}_{b k, c l}^{+}-\tilde{n}_{a m, c l}^{+} \tilde{n}_{b k, c l}^{0}\right)=0
\end{aligned}
$$

for any $(a, m),(b, k) \in H$. Let $X$ be the left-hand side in (4.6). Then $X=0$ is trivial except for the following cases:

(i) $a \sim b$ and $t_{b a} m=t_{a b} k$,

(ii) $a \sim b, p=m t_{a b}^{-1} t_{b a} \in \mathbb{Z}$, and $0<|p-k|<t_{b a}$,

(ii') $a \sim b, p^{\prime}=k t_{b a}^{-1} t_{a b} \in \mathbb{Z}$, and $0<\left|p^{\prime}-m\right|<t_{a b}$,

(iii) $a \sim b, p=m t_{a b}^{-1} t_{b a} \in \mathbb{Z}$, and $|p-k|=t_{b a}$,

(iii') $a \sim b, p^{\prime}=k t_{b a}^{-1} t_{a b} \in \mathbb{Z}$, and $\left|p^{\prime}-m\right|=t_{a b}$.

Moreover, the cases (ii') and (iii') reduce to the cases (ii) and (iii), respectively, since the left-hand side in (4.6) is skew-symmetric under $a m \leftrightarrow b k$. For the case (i), we have

$$
\begin{aligned}
X= & 2 t_{b a}^{-1}\left|c_{b a}\right|\left[t_{a b}-1\right]_{z_{a}}+t_{a b}^{-1}\left|c_{a b}\right|\left[t_{b a}\right]_{z_{b}} \cdot[2]_{z_{b}} \\
& -[2]_{z_{a}} \cdot t_{b a}^{-1}\left|c_{b a}\right|\left[t_{a b}\right]_{z_{a}}-2 t_{a b}^{-1}\left|c_{a b}\right|\left[t_{b a}-1\right]_{z_{b}} \\
= & t_{a b}^{-1}\left|c_{a b}\right|\left(\left[t_{b a}+1\right]_{z_{b}}-\left[t_{b a}-1\right]_{z_{b}}\right)-t_{b a}^{-1}\left|c_{b a}\right|\left(\left[t_{a b}+1\right]_{z_{a}}-\left[t_{a b}-1\right]_{z_{a}}\right) \\
= & t_{a b}^{-1}\left|c_{a b}\right|\left(z^{t_{b a} c_{b}}+z^{-t_{b a} c_{b}}\right)-t_{b a}^{-1}\left|c_{b a}\right|\left(z^{t_{a b} c_{a}}+z^{-t_{a b} c_{a}}\right) \\
= & 0 .
\end{aligned}
$$

Here, we use $[n]_{z} \cdot[2]_{z}=[n+1]_{z}+[n-1]_{z}$ to derive the second equality. For the case (ii), we have

$$
\begin{aligned}
X= & t_{a b}^{-1}\left|c_{a b}\right|\left[t_{b a}-|p-k|\right]_{z_{b}} \cdot[2]_{z_{b}} \\
& -t_{a b}^{-1}\left|c_{a b}\right|\left(\left[t_{b a}-|p-k|-1\right]_{z_{b}}+\left[t_{b a}-|p-k|+1\right]_{z_{b}}\right) \\
= & 0 .
\end{aligned}
$$

For the case (iii), we have

$$
X=t_{a b}^{-1}\left|c_{a b}\right|[1]_{z^{b}}-t_{b a}^{-1}\left|c_{b a}\right|[1]_{z^{a}}=0
$$

Theorem 4.12. Let $N_{0}, N_{+}, N_{-} \in \operatorname{Mat}_{H \times H}(\mathbb{Z}[z])$ be the matrices defined by

$$
N_{\varepsilon}=\left(z^{c_{a}} \tilde{n}_{a m, b k}^{\varepsilon}\right)_{a m, b k \in H} \quad(\varepsilon \in\{0,+,-\}) .
$$

Then the triple $\left(N_{0}, N_{+}, N_{-}\right)$and the pair $\left(A_{+}, A_{-}\right)=\left(N_{0}-N_{+}, N_{0}-N_{-}\right)$satisfy

(1) the conditions (N1), (N2), and (N4),

(2) the symplectic relation $A_{+} A_{-}^{\dagger}=A_{-} A_{+}^{\dagger}$, 
(3) and the condition (N3) if and only if

$$
c_{a b} \mid c_{b a} \text { or } c_{b a} \mid c_{a b} \text { for any } 1 \leq a, b \leq n .
$$

Consequently, for any Cartan matrix $C$ satisfies the condition (4.7) and any integer $\ell$ greater than or equal to 2 , the triple $\left(A_{+}, A_{-}, I_{H}\right)$ is a $T$-datum of size $|H|$.

Proof. The conditions (N1), (N2), and (N4) are obvious from the definition. The symplectic relation follows from Lemma 4.11 and the fact that $\tilde{n}_{a m, b k}^{\varepsilon}$ are invariant under $z \mapsto z^{-1}$. The condition (N3) is equivalent to $\left(t_{b a}-1\right) c_{b}<c_{a}$ for any $a, b$ such that $a \sim b$, and this is equivalent to $\operatorname{lcm}\left(c_{a}, c_{b}\right)<c_{a}+c_{b}$ for any $a, b$ such that $a \sim b$. This happens if and only if $c_{a} \mid c_{b}$ or $c_{b} \mid c_{a}$ for any $a, b$ such that $a \sim b$, and this is equivalent to the condition (4.7).

Remark 4.13. If the Cartan matrix in Theorem 4.12 and its symmetrizer satisfy the condition

$$
c_{a b}<-1 \Rightarrow c_{a}=-c_{b a}=1 \text {, }
$$

which implies (4.7), the mutation loop corresponding to the T-datum $\left(A_{+}, A_{-}, I_{H}\right)$ is explicitly constructed in [42]. The T-system associated with this T-datum is a certain truncation a T-system associated with Kirillov-Reshetikhin modules of the quantum affinization of a quantum Kac-Moody algebra [25, 35] (a truncation and a quantum Kac-Moody algebra are associated with $\ell$ and $C$, respectively).

\section{PERIOdiC Y/T-Systems}

\subsection{Finite type T-data.}

Definition 5.1. We say that a T-datum $\alpha$ is of finite type if the set $\left\{T_{a}(u) \in \mathcal{T}^{\circ}(\alpha, R, Y) \mid(a, u) \in R\right\}$ is a finite set.

Definition 5.1 does not depend on $R$ since the set being considered is a finite set for some $R$ if and only if it is a finite set for $[1, r] \times \mathbb{Z}$ by (R3). We will see that this is also independent of the choice of $Y$.

Definition 5.2. Let $\alpha$ be a T-datum and $R$ be a consistent subset for $\alpha$.

(1) We define $Y_{\text {prin }}(\alpha, R)$ to be the solution of the Y-system associated with $(\alpha, R)$ in $\operatorname{Trop}\left(u_{a, p}\right)_{(a, p) \in R_{\text {in }}}$ such that $u_{a, p}=y_{a, p}$ for any $(a, p) \in R_{\text {in }}$, where $y_{a, p}$ is defined by (3.11). By Theorem 3.19, the T-algebra $\mathcal{T}^{\circ}(\alpha, R, Y)$ is embedded into the cluster algebra with principal coefficients (see [14] for the definition of cluster algebras with principal coefficients).

(2) We define $Y_{\text {univ }}(\alpha, R)$ to be the solution of the Y-system associated with $(\alpha, R)$ in $\mathbb{Q}_{\text {sf }}\left(u_{a, p}\right)_{(a, p) \in R_{\text {in }}}$ such that $u_{a, p}=y_{a, p}$ for any $(a, p) \in R_{\text {in }}$, where $y_{a, p}$ is defined by (3.11).

Definition 5.3. Let $\alpha$ be a T-datum and $R$ be a consistent subset for $\alpha$. Let $\Omega$ be a integer with $t \mid \Omega$, where $t$ is the integer in (R3) in Definition 3.3.

(1) We say that a solution $\left(Y_{a}(u)\right)_{(a, u) \in R}$ of the Y-system associated with $(\alpha, R)$ is periodic with period $\Omega$ if $Y_{a}(u)=Y_{a}(u+\Omega)$ for any $(a, u) \in R$.

(2) We say that the T-system associated with $(\alpha, R, Y)$ is periodic with period $\Omega$ if $Y$ is periodic with period $\Omega$ and $T_{a}(u)=T_{a}(u+\Omega)$ in $\mathcal{T}^{\circ}(\alpha, R, Y)$ for any $(a, u) \in R$.

Definition 5.3 also does not depend on $R$. By the synchronicity phenomenon in cluster algebras [43], we have the following assertion:

Theorem 5.4. Let $\alpha$ be a T-datum and $R$ be a consistent subset for $\alpha$. Let $\Omega$ be a integer with $t \mid \Omega$, where $t$ is the integer in (R3) in Definition 3.3. Then the following conditions are equivalent:

(1) The T-system associated with $(\alpha, R, Y)$ is periodic with period $\Omega$ for some $Y$.

(2) The T-system associated with $(\alpha, R, Y)$ is periodic with period $\Omega$ for any $Y$.

(3) $Y_{\text {prin }}(\alpha, R)$ is periodic with period $\Omega$.

(4) $Y_{\text {univ }}(\alpha, R)$ is periodic with period $\Omega$. 
Proof. This follows from Theorem 3.19 together with the synchronicity phenomenon in cluster algebras [43, Theorem 5.2 and 5.5].

It is easy to see that $\alpha$ is of finite type (for some $Y$ ) if and only if the condition (1) in Theorem 5.4 holds for some $\Omega>0$. Therefore, Theorem 5.4 implies that Definition 5.1 does not depend on $Y$.

5.2. Simultaneous positivity of finite type T-data. For any matrix $A \in \operatorname{Mat}_{r \times r}\left(\mathbb{Z}\left[z^{ \pm 1}\right]\right)$, we define $\stackrel{\AA}{A} \in \operatorname{Mat}_{r \times r}(\mathbb{Z})$ by $\stackrel{\circ}{A}=\left.A\right|_{z=1}$. For any vector $u, v \in \mathbb{R}^{r}$, we write $u>v$ and $u \geq v$ if all components of the vector $u-v$ are positive and non-negative, respectively. The following is the main theorem in this section, which gives a effective method to determine that a given T-datum is not of finite type.

Theorem 5.5. Let $\alpha=\left(A_{+}, A_{-}, D\right)$ be a T-datum. If $\alpha$ is of finite type, then there exists a vector $v>0$ such that $\AA_{+}^{\top} v>0$ and $\AA_{-}^{\top} v>0$.

Proof. Without loss of generality we can assume that $\alpha$ is indecomposable. It is sufficient to find a vector $v \geq 0$ such that $\AA_{+}^{\top} v>0$ and $\AA_{-}^{\top} v>0$ since such a vector plus a sufficiently small positive vector is a desired vector. Let $c \in[1, r]$. Let $\mathfrak{t}^{(c)}=\left(\mathfrak{t}_{a}^{(c)}(u)\right)_{(a, u) \in[1, r] \times \mathbb{Z}}$ be the family of integers defined in Section 3.5, that is, $\mathfrak{t}_{a}^{(c)}(u)$ is the minus of the lowest power of $T_{c}(0)$ in $T_{a}(u)$, where $T_{a}(u)$ is written as a Laurent polynomial in $\left(T_{c}(p)\right)_{(c, p) \in R_{\text {in }}}$. We also define the family of integers $\tilde{\mathfrak{t}}^{(c)}=\left(\tilde{\mathfrak{t}}_{a}^{(c)}(u)\right)_{(a, u) \in[1, r] \times \mathbb{Z} \text {, where }}$ $\tilde{\mathfrak{t}}_{a}^{(c)}(u)$ is the highest power of $T_{c}(0)$ in $T_{a}(u)$. By the definitions, we have $\mathfrak{t}_{a}^{(c)}(u)+\tilde{\mathfrak{t}}_{a}^{(c)}(u) \geq 0$ for any $(a, u) \in[1, r] \times \mathbb{Z}$. By Proposition 2.6 in [47], the family of integers $\tilde{\mathfrak{t}}^{(c)}$ is uniquely determined by the initial conditions

$$
\tilde{\mathfrak{t}}_{a}^{(c)}(p)= \begin{cases}1 & \text { if }(a, p)=(c, 0) \\ 0 & \text { if }(a, p) \neq(c, 0) \text { and } 0 \leq p<p_{a}\end{cases}
$$

together with the following recurrence relation for each $(a, u) \in[1, r] \times \mathbb{Z}$ :

$$
\sum_{b, p} n_{b a ; p}^{0} \tilde{\mathfrak{t}}_{b}^{(c)}(u+p)=\max \left(\sum_{b, p} n_{b a ; p}^{-} \tilde{\mathfrak{t}}_{b}^{(c)}(u+p), \sum_{b, p} n_{b a ; p}^{+} \tilde{\mathfrak{t}}_{b}^{(c)}(u+p)\right) .
$$

The family of integers $\mathfrak{t}^{(c)}$ and $\tilde{\mathfrak{t}}^{(c)}$ satisfy the same recurrence relation, but have the different initial conditions.

Let $v_{a}^{(c)}$ and $\tilde{v}_{a}^{(c)}$ be the integers defined by

$$
v_{a}^{(c)}=\sum_{u=0}^{\Omega-1} \mathfrak{t}_{a}^{(c)}(u), \quad \tilde{v}_{a}^{(c)}=\sum_{u=0}^{\Omega-1} \tilde{\mathfrak{t}}_{a}^{(c)}(u),
$$

where $\Omega$ is a period of the T-system. By the periodicity of the T-system, we have

$$
v_{a}^{(c)}=\sum_{u=0}^{\Omega-1} \mathfrak{t}_{a}^{(c)}(u+p), \quad \tilde{v}_{a}^{(c)}=\sum_{u=0}^{\Omega-1} \tilde{\mathfrak{t}}_{a}^{(c)}(u+p)
$$

for any $p \in \mathbb{Z}$. By summing up (3.16) with respect to the period, we have

$$
\begin{aligned}
\sum_{b} \grave{n}_{b a}^{0} v_{b}^{(c)} & =\sum_{u=0}^{\Omega-1} \max \left(\sum_{b, p} n_{b a ; p}^{-} \mathfrak{t}_{b}^{(c)}(u+p), \sum_{b a ; p} n_{b a ; p}^{+} \mathfrak{t}_{b}^{(c)}(u+p)\right) \\
& \geq \max \left(\sum_{b} \stackrel{\circ}{b a}_{b a}^{-} v_{b}^{(c)}, \sum_{b} \stackrel{\circ}{b a}_{b a}^{+} v_{b}^{(c)}\right),
\end{aligned}
$$


where $\grave{n}_{b a}^{\varepsilon}=\sum_{p} n_{b a, p}$. This implies that $\AA_{+}^{\top} v^{(c)} \geq 0$ and $\AA_{-}^{\top} v^{(c)} \geq 0$. Similarly, we have $\AA_{+}^{\top} \tilde{v}^{(c)} \geq 0$ and $\AA_{-}^{\top} \tilde{v}^{(c)} \geq 0$ by summing up (5.2) with respect to the period. Let $v$ and $\tilde{v}$ be the vectors defined by

$$
v=\sum_{c=1}^{r}\left[\begin{array}{c}
v_{1}^{(c)} \\
\vdots \\
v_{r}^{(c)}
\end{array}\right], \quad \tilde{v}=\sum_{c=1}^{r}\left[\begin{array}{c}
\tilde{v}_{1}^{(c)} \\
\vdots \\
\tilde{v}_{r}^{(c)}
\end{array}\right] .
$$

We then define a vector $v^{\prime}$ by $v^{\prime}=v+\tilde{v}$. We have $v^{\prime} \geq 0$ since $\mathfrak{t}_{a}^{(c)}(u)+\tilde{\mathfrak{t}}_{a}^{(c)}(u) \geq 0$. We also have $\AA_{+}^{\top} v^{\prime} \geq 0$ and $\AA_{-}^{\top} v^{\prime} \geq 0$. Therefore, if we prove that $\AA_{+}^{\top} v>0$ and $\AA_{-}^{\top} v>0$, the assertion of the theorem follows.

From (3.17), (3.19), and (5.4), the $a$-th component of $\AA_{ \pm}^{\top} v$ is positive if and only if there exists $(c, u) \in$ $[1, r] \times \mathbb{Z}$ such that $\left[ \pm \mathfrak{y}_{a}^{(c)}(u)\right]_{+} \neq 0$. Therefore the $a$-th component of $\AA_{ \pm}^{\top} v$ is positive if the $a$-th column of $N_{ \pm}$is non-zero by (3) in Lemma 3.23. It remains to prove that the $a$-th component of $\AA_{ \pm}^{\top} v$ is also positive when the $a$-th column of $N_{ \pm}$is zero. If both the $a$-th columns of $N_{+}$and $N_{-}$are zero, the assertion of the theorem follows from Corollary 3.25. Thus we can assume that either the $a$-th column of $N_{+}$or $N_{-}$ is non-zero. Without loss of generality we assume that the $a$-th column of $N_{+}$is non-zero and the $a$-th column of $N_{-}$is zero. Let $n_{c a ; p}^{+} z^{p}$ be a term in the $a$-th column of $N_{+}$with the minimal degree among the terms in this column. Now we have

$$
\begin{aligned}
{\left[-\hat{\mathfrak{y}}_{a}^{(\sigma(c))}\left(-p_{\sigma(c)}-p\right)\right]_{+} } & =\sum_{b, q}\left(n_{b a ; q}^{0}-n_{b a ; q}^{-}\right) \mathfrak{t}_{b}^{(\sigma(c))}\left(-p_{\sigma(c)}-p+q\right) \\
& =\sum_{b, q} n_{b a ; q}^{0} \mathfrak{t}_{b}^{(\sigma(c))}\left(-p_{\sigma(c)}-p+q\right) \\
& =\max \left(\sum_{b, q} n_{b a ; q}^{+} \mathfrak{t}_{b}^{(\sigma(c))}\left(-p_{\sigma(c)}-p+q\right), 0\right) \\
& =\max \left(n_{c a ; p}^{+} \mathfrak{t}_{c}^{(\sigma(c))}\left(-p_{\sigma(c)}\right), 0\right) \\
& =\max \left(n_{c a ; p}^{+}, 0\right) \\
& =n_{c a ; p}^{+},
\end{aligned}
$$

and this implies that the $a$-th component of $\AA_{-}^{\top} v$ is positive.

\section{Example 5.6.}

(1) A T-datum of size 1 (Theorem 4.1) is of finite type if and only if $\left(A_{+}, A_{-}\right)$is one of the following three pairs of matrices for some $p>0$ :

$$
\begin{array}{ll}
A_{+}=\left[1+z^{2 p}\right], & A_{-}=\left[1+z^{2 p}\right], \\
A_{+}=\left[1-z^{p}+z^{2 p}\right], & A_{-}=\left[1+z^{2 p}\right], \\
A_{+}=\left[1+z^{2 p}\right], & A_{-}=\left[1-z^{p}+z^{2 p}\right] .
\end{array}
$$

The if part is proved by direct calculations, and the only if part follows from Theorem 5.5.

(2) A T-datum associated with a bipartite recurrent quiver, which is a special case of a T-datum in Proposition 4.4, is of finite type if and only if both $A$ and $A^{\prime}$ are direct sums of $A D E$ Cartan matrices [19]. In fact, Theorem 5.5 generalizes Proposition 7.1 in [19] to arbitrary T-data.

(3) A T-datum in Example 4.7 is of finite type if and only if both $\bar{A}$ and $\bar{A}^{\prime}$ are of finite type Cartan matrices, except that one of them can be of tadpole type. The if part is proved in [33], and the only if part follows from Theorem 5.5.

(4) A T-datum in Theorem 4.12 is of finite type if and only if $C$ is of finite type Cartan matrix. The if part is proved in $[27,28]$, and the only if part follows from Proposition 4.10 and Theorem 5.5. 


\subsection{Special values of the dilogarithm function.}

Definition 5.7. Let $\alpha=\left(A_{+}, A_{-}, D\right)$ be a T-datum. Let $P=\operatorname{diag}\left(z^{-p_{a} / 2}\right)_{a \in[1, r]}$, where $p_{a}$ is the integer in (N1). We say that $\alpha$ is Cartan-like if both the matrices $P A_{+}$and $P A_{-}$are invariant under $z \mapsto z^{-1}$.

This terminology comes from the fact that T-data in Section 4.2 satisfy this property. All examples in Section 4 are also Cartan-like. Note that $\AA_{ \pm}$are not Cartan matrices in general since they may not be sign-symmetric (see examples in Table 2 and 3). The matrix $N_{0}$ in the Cartan-like T-datum should be a diagonal matrix. This property is useful due to the following fact on real square matrices whose off-diagonal entries are non-positive. As a result, we assign a positive definite symmetric matrix to any Cartan-like T-datum of finite type (Proposition 5.9).

Lemma 5.8 ([8, Theorem 4.3]). Let $A$ be a real square matrix whose off-diagonal entries are all nonpositive. Then the following conditions are equivalent:

(1) there exists $v>0$ such that $A v>0$,

(2) all real eigenvalues of $A$ are positive.

Proposition 5.9. Let $\alpha=\left(A_{+}, A_{-}, D\right)$ is a Cartan-like T-datum of finite type. Then the following assertions hold:

(1) $\AA_{+}$and $\AA_{-}$are invertible.

(2) Let $K=\left(\kappa_{a b}\right)_{a, b \in[1, r]}$ be the matrix defined by $K=\AA_{+}^{-1} \AA_{-}$. Then $K D$ is a positive definite symmetric matrix.

(3) Let $K^{\vee}=\left(\check{\kappa}_{a b}\right)_{a, b \in[1, r]}$ be the matrix defined by $K^{\vee}=D^{-1} K D$. Then $K^{\vee} D^{\vee}$ is a positive definite symmetric matrix.

Proof. By Theorem 5.5 and Lemma 5.8, all the real eigenvalues of $\AA_{ \pm}$are positive. This implies (1). Since $K^{\vee}=\left(\AA_{+}^{\vee}\right)^{-1} \AA_{-}^{\vee}$, the assertion (3) follows from the assertion (2) for $\alpha^{\vee}$. We now prove (2). We first see that $K$ is symmetric due to the symplectic relation. Suppose that there exists an eigenvector $v$ of $K$ with a non-positive eigenvalue. Let us denote by $-\lambda$ this eigenvalue. Then we have $\left(\AA_{-}+\lambda \AA_{+}\right) v=0$. Thus 0 is an eigenvalue of $\AA_{-}+\lambda \AA_{+}$. Since $\lambda \geq 0$, all off-diagonal entries in $\AA_{-}+\lambda \AA_{+}$are non-positive. Moreover, this matrix satisfies the condition (1) in Lemma 5.8 by Theorem 5.5. Thus its real eigenvalues are positive by Lemma 5.8, a contradiction.

The function

$$
\operatorname{Li}_{2}(z):=\sum_{n=1}^{\infty} \frac{z^{n}}{n^{2}} \quad(|z|<1)
$$

is called the dilogarithm function. The Rogers dilogarithm function is a function on the interval $(0,1)$ defined as follows:

$$
L(x)=\mathrm{Li}_{2}(x)+\frac{1}{2} \log (x) \log (1-x) .
$$

We can define $L(0)=0$ and $L(1)=\pi^{2} / 6$ by continuity.

For any T-datum $\alpha=\left(A_{+}, A_{-}, D\right)$, we denote by $d_{a}$ and $d_{a}^{\vee}$ the $a$-th entries in $D$ and $D^{\vee}$, respectively.

Theorem 5.10. Let $\alpha=\left(A_{+}, A_{-}, D\right)$ be a Cartan-like T-datum of finite type. Let $K^{\vee}=\left(\check{\kappa}_{a b}\right)_{a, b \in[1, r]}$ be the matrix defined in Proposition 5.9.

(1) The system of equations

$$
f_{a}=\prod_{b=1}^{r}\left(1-f_{b}\right)^{\check{\kappa}_{a b}} \quad(a \in[1, r])
$$

has a unique real solution such that $0<f_{a}<1$ for any $a \in[1, r]$. 


\begin{tabular}{|c|c|c|c|}
\hline$A_{+}$ & $A_{-}$ & $K$ & $c_{\alpha}$ \\
\hline$\left[\begin{array}{cc}1+z^{2} & -z \\
-z & 1+z^{2}\end{array}\right]$ & {$\left[\begin{array}{cc}1+z^{2} & 0 \\
0 & 1+z^{2}\end{array}\right]$} & {$\left[\begin{array}{ll}4 / 3 & 2 / 3 \\
2 / 3 & 4 / 3\end{array}\right]$} & $4 / 5$ \\
\hline$\left[\begin{array}{cc}1+z^{2} & -z \\
-z & 1+z^{2}\end{array}\right]$ & {$\left[\begin{array}{cc}1-z+z^{2} & 0 \\
0 & 1-z+z^{2}\end{array}\right.$} & {$\left[\begin{array}{ll}2 / 3 & 1 / 3 \\
1 / 3 & 2 / 3\end{array}\right]$} & 1 \\
\hline$\left[\begin{array}{cc}1+z^{2} & -z \\
-z-z^{5} & 1+z^{6}\end{array}\right]$ & {$\left[\begin{array}{cc}1+z^{2} & 0 \\
-z^{3} & 1+z^{6}\end{array}\right]$} & {$\left[\begin{array}{cc}3 / 2 & 1 \\
1 & 2\end{array}\right]$} & $5 / 7$ \\
\hline$\left[\begin{array}{cc}1+z^{2} & -z \\
-z-z^{2} & 1+z^{3}\end{array}\right]$ & {$\left[\begin{array}{cc}1-z+z^{2} & 0 \\
0 & 1+z^{3}\end{array}\right]$} & {$\left[\begin{array}{ll}1 & 1 \\
1 & 2\end{array}\right]$} & $3 / 4$ \\
\hline$\left[\begin{array}{cc}1+z^{2} & -z \\
-z-z^{5}-z^{9} & 1+z^{10}\end{array}\right]$ & {$\left[\begin{array}{cc}1+z^{2} & 0 \\
-z^{3}-z^{7} & 1+z^{10}\end{array}\right]$} & {$\left[\begin{array}{ll}2 & 2 \\
2 & 4\end{array}\right]$} & $4 / 7$ \\
\hline
\end{tabular}

TABle 2. Examples of Cartan-like T-data of finite type of size 2, where $D=I_{2}$ in these examples.

(2) Let $\left(f_{a}\right)_{a \in[1, r]}$ be the unique solution in (1). Define the real number $c_{\alpha}$ by

$$
c_{\alpha}:=\frac{6}{\pi^{2}} \sum_{a=1}^{r} d_{a} L\left(f_{a}\right) .
$$

Then we have $c_{\alpha} \in \mathbb{Q}$.

Proof. We define a function $F_{\alpha}(x):[0, \infty)^{r} \rightarrow \mathbb{R}$ by

$$
F_{\alpha}(x)=\frac{1}{2} x^{\top} K^{\vee} D^{\vee} x+\sum_{a=1}^{r}\left(d_{a}^{\vee}\right)^{-1} \operatorname{Li}_{2}\left(\exp \left(-d_{a}^{\vee} x_{a}\right)\right) .
$$

By setting $f_{a}=1-\exp \left(-d_{a}^{\vee} x_{a}\right)$, we see that the statement (1) is equivalent to saying that the function $F_{\alpha}(x)$ has a unique critical point in $(0, \infty)^{r}$. This follows from the fact that $K^{\vee} D^{\vee}$ is a positive definite symmetric matrix, as in the proof of Lemma 2.1 in [49].

We now prove (2). From the result in [41, Section 6], the value

$$
\frac{6}{\pi^{2}} \sum_{\substack{(a, u) \in[1, r] \times \mathbb{Z} \\ 0 \leq u<\Omega}} d_{a} L\left(\frac{Y_{a}(u)}{1 \oplus Y_{a}(u)}\right)
$$

is an integer for any solution $Y=\left(Y_{a}(u)\right)_{(a, u) \in[1, r] \times \mathbb{Z}}$ of the $\mathrm{Y}$-system associated with $(\alpha,[1, r] \times \mathbb{Z})$ in the semifield $\mathbb{R}_{>0}$, where $\Omega>0$ is a period of $Y_{\text {univ }}(\alpha,[1, r] \times \mathbb{Z})$. Moreover, this value is independent of the choice of $Y$. In fact, Nakanishi [41] proved that these facts follow from the sign coherence property of cluster algebras, which was proved by Gross, Hacking, Keel, and Kontsevich [23] for skew-symmetrizable cluster algebras.

It is easy to see that the system of equations (5.5) is equivalent to

$$
\prod_{b=1}^{r} f_{b}^{\sum_{p \in \mathbb{Z}}\left(\check{n}_{a b ; p}^{0}-\check{n}_{a b ; p}^{+}\right)}=\prod_{b=1}^{r}\left(1-f_{b}\right)^{\sum_{p \in \mathbb{Z}}\left(\check{n}_{a b ; p}^{0}-\check{n}_{b a ; p}^{-}\right)} \quad(a \in[1, r]) .
$$

Thus the family $Y=\left(Y_{a}(u)\right)_{(a, u) \in[1, r] \times \mathbb{Z}}$ defined by $Y_{a}(u)=f_{a} /\left(1-f_{a}\right)$ is a solution of the Y-system associated with $(\alpha,[1, r] \times \mathbb{Z})$ in $\mathbb{R}_{>0}$. Since this is a constant solution with respect to $u$, the integer (5.6) is equal to $\Omega c_{\alpha}$. Thus $c_{\alpha}$ is a rational number.

Example 5.11. We give some examples of Cartan-like T-data of finite type of size 2 and size 3 in Table 2 and 3 , respectively, where the matrix $D$ in these examples are the identity matrices. We also show 


\begin{tabular}{|c|c|c|c|c|c|c|c|c|c|}
\hline$A_{+}$ & & $A_{-}$ & & & & $K$ & & & $c_{\alpha}$ \\
\hline$\left\lceil 1+z^{2}\right.$ & $-z$ & {$\left[1+z^{2}\right.$} & 0 & 0 & & {$[3 / 2$} & 1 & $1 / 2]$ & \\
\hline$-z$ & $1+z^{2} \quad-z$ & 0 & $1+z^{2}$ & 0 & & 1 & 2 & 1 & 1 \\
\hline 0 & $\left.\begin{array}{ll}-z & 1+z^{2}\end{array}\right]$ & [ 0 & 0 & $\left.1+z^{2}\right\rfloor$ & & $1 / 2$ & 1 & $3 / 2$ & \\
\hline$\left[1+z^{2}\right.$ & $-z$ & {$[1-z+z$} & $z^{2}$ & 0 & 0 & {$[3 / 4$} & $1 / 2$ & $1 / 47$ & \\
\hline$-z$ & $1+z^{2} \quad-z$ & 0 & $1-z$ & $+z^{2}$ & 0 & $1 / 2$ & 1 & $1 / 2$ & $9 / 7$ \\
\hline 0 & $\left.\begin{array}{ll}-z & 1+z^{2}\end{array}\right]$ & 0 & & 0 & $\left.1-z+z^{2}\right\rfloor$ & $1 / 4$ & $1 / 2$ & $3 / 4$ & \\
\hline$\left[1+z^{2}\right.$ & $-z$ & {$[1-z+z$} & $z^{2}$ & 0 & 07 & $\lceil 1$ & $\left.\begin{array}{ll}1 & 1\end{array}\right]$ & & \\
\hline$-z$ & $1+z^{2}$ & 0 & $1-z$ & $+z^{2}$ & 0 & 1 & $2 \quad 2$ & & $9 / 10$ \\
\hline L 0 & $-z-z^{2} \quad 1+z^{3}$ & 0 & & 0 & $\left.1+z^{3}\right\rfloor$ & 1 & $\left.\begin{array}{ll}2 & 3\end{array}\right]$ & & \\
\hline$\left[1+z^{2}\right.$ & $-z$ & {$\left[1+z^{2}\right.$} & $-z$ & 0 & & 2 & $\left.\begin{array}{ll}0 & 2\end{array}\right]$ & & \\
\hline$-z^{3}$ & $1+z^{6}$ & $-z-z^{5}$ & $1+z^{6}$ & 0 & & 0 & $\begin{array}{ll}1 & 1\end{array}$ & & 1 \\
\hline$\left[-z-z^{7}\right.$ & $-z^{2}-z^{6} \quad 1+z^{8}$ & 0 & 0 & $1+z$ & & 2 & $\left.\begin{array}{ll}1 & 4\end{array}\right]$ & & \\
\hline$\left[1+z^{2}\right.$ & $-z$ & {$\left[1+z^{2}\right.$} & 0 & 0 & & {$[2$} & $\left.\begin{array}{ll}2 & 2\end{array}\right]$ & & \\
\hline$-z$ & $1+z^{2}$ & 0 & $1+z^{2}$ & 0 & & 2 & $4 \quad 4$ & & $2 / 3$ \\
\hline 0 & $\left.1-z+z^{2}\right\rfloor$ & [ & 0 & $\left.1+z^{2}\right]$ & & 2 & $\left.\begin{array}{ll}4 & 6\end{array}\right]$ & & \\
\hline$\left[1+z^{2}\right.$ & $-z$ & {$\left[1+z^{2}\right.$} & 0 & 0 & & {$[2$} & $\left.\begin{array}{ll}2 & 1\end{array}\right]$ & & \\
\hline$-z-z^{5}$ & $1+z^{6} \quad-z^{3}$ & $-z^{3}$ & $1+z^{6}$ & 0 & & 2 & $4 \quad 2$ & & $4 / 5$ \\
\hline [ 0 & $\begin{array}{ll}-z^{3} & 1+z^{6}\end{array}$ & 0 & 0 & $\left.1+z^{6}\right]$ & & 1 & $\left.\begin{array}{ll}2 & 2\end{array}\right]$ & & \\
\hline$\left[1-z+z^{2}\right.$ & $-z$ & $1+z^{2}$ & 0 & & 0 & 4 & 2 & -17 & \\
\hline$-z$ & $1+z^{2}$ & 0 & $1+z$ & & $-z$ & 2 & 2 & -1 & $3 / 2$ \\
\hline L 0 & $\left.1+z^{5}\right]$ & $-z^{2}-z^{3}$ & $-z-z$ & & $+z^{5}$ & {$[-1$} & -1 & $1]$ & \\
\hline
\end{tabular}

TABle 3. Examples of Cartan-like T-data of finite type of size 3, where $D=I_{3}$ in these examples.

the positive definite symmetric matrix $K$ and the rational number $c_{\alpha}$ associated with these T-data. The rational number $c_{\alpha}$ can be computed by using Theorem 6.8 in [41].

5.4. Partition $q$-series. Let $\alpha=\left(A_{+}, A_{-}, D\right)$ be a Cartan-like T-datum of finite type of size $r$. We define two sets $H_{\alpha}$ and $H_{\alpha}^{\prime}$ by

$$
\begin{aligned}
& H_{\alpha}=\left\{(m, l) \in \mathbb{Z}^{r} \times \mathbb{Q}^{r} \mid \AA_{-} m=\AA_{+} l\right\}, \\
& H_{\alpha}^{\prime}=\left\{\left(\left(\AA_{+}^{\vee}\right)^{\top} n,\left(\AA_{-}^{\vee}\right)^{\top} n\right) \mid n \in \mathbb{Z}^{r}\right\} .
\end{aligned}
$$

These are free abelian groups of rank $r$, and the symplectic relation implies that $H_{\alpha}^{\prime}$ is a subgroup of $H_{\alpha}$. Let $S_{\alpha}$ be the quotient group of $H_{\alpha}$ by $H_{\alpha}^{\prime}: S_{\alpha}=H_{\alpha} / H_{\alpha}^{\prime}$. This is a finite abelian group that is isomorphic to $\mathbb{Z}^{r} /\left(\right.$ the rows space of $\left.\AA_{+}^{\vee}\right)$. In particular, the order of $S_{\alpha}$ is $\operatorname{det} \AA_{+}$. For any $\sigma \in S_{\alpha}$, we denote by $\sigma \geq 0$ the set $\{(m, l) \in \sigma \mid m \geq 0\}$.

Definition 5.12. Let $\alpha=\left(A_{+}, A_{-}, D\right)$ be a Cartan-like T-datum of finite type. Let $\sigma \in S_{\alpha}$. We define the partition $q$-series of $\alpha$ at $\sigma$ by

$$
\mathcal{Z}_{\alpha, \sigma}(q):=\sum_{(m, l) \in \sigma \geq 0} \frac{q^{\frac{1}{2}\langle m, l\rangle}}{\prod_{a=1}^{r}\left(q^{d_{a}^{\vee}}\right)_{m_{a}}},
$$

where $\langle m, l\rangle:=m^{\top} D^{\vee} l$ and $(q)_{n}=\prod_{i=1}^{n}\left(1-q^{i}\right)$ is the $q$-Pochhammer symbol. We also define the total partition q-series of $\alpha$ by

$$
\mathcal{Z}_{\alpha, \text { tot }}(q):=\sum_{\sigma \in S_{\alpha}} \mathcal{Z}_{\alpha, \sigma}(q)=\sum_{m \in\left(\mathbb{Z}_{\geq 0}\right)^{r}} \frac{q^{\frac{1}{2} m^{\top} K^{\vee} D^{\vee} m}}{\prod_{a=1}^{r}\left(q^{d_{a}^{\vee}}\right)_{m_{a}}} .
$$

Proposition 5.13. (1) The partition $q$-series $\mathcal{Z}_{\alpha, \sigma}(q)$ with $q=e^{2 \pi i \tau}$ converges to a holomorphic function on the upper half plane $\mathbb{H}=\{\tau \in \mathbb{C} \mid \operatorname{Im} \tau>0\}$, where we set $q^{\kappa}=e^{2 \pi i \tau \kappa}$ for any $\kappa \in \mathbb{Q}$. 
(2) We have

$$
\lim _{\varepsilon \searrow 0} \varepsilon \log \mathcal{Z}_{\alpha, \text { tot }}\left(e^{-\varepsilon}\right)=\frac{\pi^{2}}{6 \delta} c_{\alpha},
$$

where $\delta=\operatorname{lcm}\left(d_{1}, \ldots, d_{r}\right) \operatorname{gcd}\left(d_{1}, \ldots, d_{r}\right)$ and $c_{\alpha}$ is the rational number in Theorem 5.10.

Proof. (1) follows from the fact that $K^{\vee} D^{\vee}$ is a positive definite symmetric matrix (Proposition 5.9). (2) follows from the asymptotic analysis in [49].

Let $\Gamma \subseteq \mathrm{SL}(2, \mathbb{Z})$ be a congruence subgroup. We say that a holomorphic function $f(\tau)$ on the upper half plane is a modular function with respect to $\Gamma$ if $f(\tau)=f\left(\frac{a \tau+b}{c \tau+d}\right)$ for any $\tau \in \mathbb{H}$ and $\left[\begin{array}{ll}a & b \\ c & d\end{array}\right] \in \Gamma$, and $f(\tau)$ is meromorphic at each cusp of $\Gamma$.

Conjecture 5.14. Let $\alpha=\left(A_{+}, A_{-}, D\right)$ be a Cartan-like T-datum of finite type. Then there exists a congruence subgroup $\Gamma \subseteq \mathrm{SL}(2, \mathbb{Z})$ such that $q^{-c_{\alpha} / 24} \mathcal{Z}_{\alpha, \sigma}(q)$ with $q=e^{2 \pi i \tau}$ is a modular function with respect to $\Gamma$ for any $\sigma \in S_{\alpha}$, where $c_{\alpha}$ is the rational number in Theorem 5.10 .

Remark 5.15. For any solution $\left(f_{a}\right)_{a \in[1, r]} \in \overline{\mathbb{Q}}^{r}$ of $(5.5)$, we can define the element

$$
\sum_{a=1}^{r} d_{a}\left[f_{a}\right] \in \mathcal{B}(F)
$$

where $F$ is a number field containing the solution, and $\mathcal{B}(F)$ is the Bloch group of $F$. By the result in [41, Section 6], we see that the element (5.7) is a torsion (see [37]). Conjecture 5.14 can be regarded as a version of Nahm's Conjecture [40, 50], which relates torsions in Bloch groups and the modularity of $q$-hypergeometric series.

Theorem 5.16. Conjecture 5.14 holds for $r=1$.

Proof. From (1) in Example 5.6, it is sufficient to prove the following three cases:

$$
\begin{aligned}
& \alpha_{1}=\left(1+z^{2 p}, 1+z^{2 p}, d\right), \\
& \alpha_{2}=\left(1-z^{p}+z^{2 p}, 1+z^{2 p}, d\right), \\
& \alpha_{3}=\left(1+z^{2 p}, 1-z^{p}+z^{2 p}, d\right) .
\end{aligned}
$$

For these three cases, we have $S_{\alpha_{1}} \cong \mathbb{Z} / 2 \mathbb{Z}, S_{\alpha_{2}} \cong 0$, and $S_{\alpha_{3}} \cong \mathbb{Z} / 2 \mathbb{Z}$. We also have $L_{\alpha_{1}}=d / 2$, $L_{\alpha_{2}}=2 d / 5$, and $L_{\alpha_{3}}=3 d / 5$.

We first consider $\alpha_{2}$ because its proof is the simplest and follows from a well-known discussion (e.g., see [50, Chapter II, Section 3]). In this case, the partition $q$-series is given by

$$
\mathcal{Z}_{\alpha_{2}, 0}(q)=\sum_{n \in \mathbb{Z}_{\geq 0}} \frac{q^{d n^{2}}}{\left(q^{d}\right)_{n}}
$$

Using the Rogers-Ramanujan identity

$$
\sum_{n=0}^{\infty} \frac{q^{n^{2}}}{(q)_{n}}=\prod_{\substack{n>0 \\ n \equiv \pm 1}} \frac{1}{1-q^{n}}
$$

together with the Jacobi triple product identity, we have

$$
q^{-d / 60} \mathcal{Z}_{\gamma}(q)=\frac{1}{2 \eta\left(q^{d}\right)} \sum_{n \in \mathbb{Z}} a(n) q^{d n^{2} / 40}
$$


where $\eta(q)=q^{1 / 24} \prod_{n=1}^{\infty}\left(1-q^{n}\right)$ is the Dedekind eta, and

$$
a(n)=\left\{\begin{array}{lll}
1 & \text { if } n \equiv \pm 1 \quad(\bmod 20) \\
-1 & \text { if } n \equiv \pm 9 \\
0 & \text { otherwise }
\end{array} \quad(\bmod 20)\right.
$$

Since the right-hand side in (5.8) is the ratio of modular forms of weight $1 / 2$, it is a modular function. Thus we obtain the assertion for $\alpha_{2}$.

We now prove the assertion for $\alpha_{1}$ and $\alpha_{3}$. The partition $q$-series in these cases are given by

$$
\begin{aligned}
& \mathcal{Z}_{\alpha_{3}, 0}(q)=\sum_{n \in \mathbb{Z}_{\geq 0}} \frac{q^{d n^{2}}}{\left(q^{d}\right)_{2 n}}, \quad \mathcal{Z}_{\alpha_{3}, 1}(q)=\sum_{n \in \mathbb{Z}_{\geq 0}} \frac{q^{d\left(n^{2}+n+\frac{1}{4}\right)}}{\left(q^{d}\right)_{2 n+1}}, \\
& \mathcal{Z}_{\alpha_{1}, 0}(q)=\sum_{n \in \mathbb{Z}_{\geq 0}} \frac{q^{2 d n^{2}}}{\left(q^{d}\right)_{2 n}}, \quad \mathcal{Z}_{\alpha_{1}, 1}(q)=\sum_{n \in \mathbb{Z}_{\geq 0}} \frac{q^{d\left(2 n^{2}+2 n+\frac{1}{2}\right)}}{\left(q^{d}\right)_{2 n+1}} .
\end{aligned}
$$

To prove the assertion for $\alpha_{1}$ and $\alpha_{3}$, we use the following RogersRamanujan type identities (see [39, S. 98, S. 94, S. 83, and S. 86] and references therein):

$$
\begin{aligned}
& \sum_{n=0}^{\infty} \frac{q^{n^{2}}}{(q)_{2 n}}=\prod_{\substack{n>0 \\
n \equiv \pm 1, \pm 3, \pm 4, \pm 5, \pm 7, \pm 9(\bmod 20)}} \frac{1}{1-q^{n}} \\
& \sum_{n=0}^{\infty} \frac{q^{n^{2}+n}}{(q)_{2 n+1}}=\prod_{\substack{n>0 \\
n \equiv \pm 1, \pm 2, \pm 5, \pm 6, \pm 8, \pm 9(\bmod 20)}} \frac{1}{1-q^{n}} \\
& \sum_{n=0}^{\infty} \frac{q^{2 n^{2}}}{(q)_{2 n}}=\prod_{\substack{n>0 \\
n \equiv \pm 2, \pm 3, \pm 4, \pm 5(\bmod 16)}} \frac{1}{1-q^{n}} \\
& \sum_{n=0}^{\infty} \frac{q^{2 n^{2}+2 n}}{(q)_{2 n+1}}=\prod_{\substack{n>0 \\
n \equiv \pm 1, \pm 4, \pm 6, \pm 7}} \frac{1}{1-q^{n}}
\end{aligned}
$$

Using (5.9) and (5.10) together with the quintuple product identity, we have

$$
q^{-d / 40} \mathcal{Z}_{\alpha_{3}, \sigma}(q)=\frac{1}{2 \eta\left(q^{d}\right)} \sum_{n \in \mathbb{Z}} a_{3, \sigma}(n) q^{d n^{2} / 60}
$$

where

$$
\begin{aligned}
& a_{3,0}(n)= \begin{cases}1 & \text { if } n \equiv \pm 1 \quad(\bmod 30), \\
-1 & \text { if } n \equiv \pm 11 \quad(\bmod 30) \\
0 & \text { otherwise }\end{cases} \\
& a_{3,1}(n)=\left\{\begin{array}{lll}
1 & \text { if } n \equiv \pm 4 \quad(\bmod 30), \\
-1 & \text { if } n \equiv \pm 14 \quad(\bmod 30), \\
0 & \text { otherwise. }
\end{array}\right.
\end{aligned}
$$

Thus we obtain the assertion for $\alpha_{3}$. Similarly, using (5.11) and (5.12) together with the quintuple product identity, we have

$$
q^{-d / 48} \mathcal{Z}_{\alpha_{1}, \sigma}(q)=\frac{1}{2 \eta\left(q^{d}\right)} \sum_{n \in \mathbb{Z}} a_{1, \sigma}(n) q^{d n^{2} / 48}
$$


where

$$
\begin{aligned}
& a_{1,0}(n)= \begin{cases}1 & \text { if } n \equiv \pm 1 \quad(\bmod 24), \\
-1 & \text { if } n \equiv \pm 7 \quad(\bmod 24), \\
0 & \text { otherwise }\end{cases} \\
& a_{1,1}(n)= \begin{cases}1 & \text { if } n \equiv \pm 5 \quad(\bmod 24), \\
-1 & \text { if } n \equiv \pm 11 \quad(\bmod 24), \\
0 & \text { otherwise }\end{cases}
\end{aligned}
$$

Thus we obtain the assertion for $\alpha_{1}$.

We give some examples supporting Conjecture 5.14 for $r \geq 2$.

Example 5.17 (Zagier's lists). Any $2 \times 2$ or $3 \times 3$ matrix $K$ for the Cartan-like T-data in Table 2 and 3 appears in lists of Zagier [50, Table 2 and 3] as an example where

$$
\sum_{m \in\left(\mathbb{Z}_{\geq 0}\right)^{r}} \frac{q^{\frac{1}{2} m^{\top} K m+B^{\top} m+C}}{\prod_{a=1}^{r}(q)_{m_{a}}}
$$

appears to be a modular function for some $B \in \mathbb{Q}^{r}$ and $C \in \mathbb{Q}$. We can see that all sporadic examples with $B=0$ in his lists are obtained from $\left(A_{+}, A_{-}\right)$or $\left(A_{-}, A_{+}\right)$in our Table 2 and 3.

Example 5.18 (Andrew-Gordon identity). Let $\alpha$ be the Cartan-like T-datum associated with the tadpole type $T_{r}$ (see Example 4.8). It is of finite type since its T-system can be obtained from the T-system associated with the bipartite belt of type $A_{2 r}$, which is periodic, by an identification of variables. Since $\operatorname{det} \AA_{+}=1$, we have $S_{\alpha}=0$. By using Theorem 6.1 in [41], we see that the rational number $c_{\alpha}$ is given by $c_{\alpha}=1-3 /(2 r+3)$. The partition $q$-series of $\alpha$ is given by

$$
\mathcal{Z}_{\alpha, 0}(q)=\sum_{n \in\left(\mathbb{Z}_{\geq 0}\right)^{r}} \frac{q^{N_{1}^{2}+\cdots+N_{r}^{2}}}{(q)_{n_{1}} \cdots(q)_{n_{r}}}
$$

where $N_{a}=n_{a}+\cdots+n_{r}$. Using the Andrew-Gordon identity [1]

$$
\sum_{n \in\left(\mathbb{Z}_{\geq 0}\right)^{r}} \frac{q^{N_{1}^{2}+\cdots+N_{r}^{2}}}{(q)_{n_{1}} \cdots(q)_{n_{r}}}=\prod_{\substack{n>0 \\ n \neq 0, \pm(r+1)(\bmod 2 r+3)}} \frac{1}{1-q^{n}},
$$

together with the Jacobi triple product identity, we have

$$
q^{-c_{\alpha} / 24} \mathcal{Z}_{\alpha, 0}(q)=\frac{1}{2 \eta(q)} \sum_{n \in \mathbb{Z}} a(n) q^{n^{2} /(8(2 r+3))}
$$

where

$$
a(n)= \begin{cases}1 & \text { if } n \equiv \pm 1 \quad(\bmod 4(2 r+3)) \\ -1 & \text { if } n \equiv \pm(4 r+5) \quad(\bmod 4(2 r+3)), \\ 0 & \text { otherwise }\end{cases}
$$

This implies that $q^{-c_{\alpha} / 24} \mathcal{Z}_{\alpha, 0}(q)$ is a modular function.

Example 5.19 (Fermionic formulas). For any quantum affine algebra $U_{q}(\hat{\mathfrak{g}})$ and positive integer with $\ell \geq 2$, the level $\ell$ restricted T-system and Y-system for $U_{q}(\hat{\mathfrak{g}})$ are defined (see [36]). Reading the exponents in the T-system and Y-system in [36, Section 2], we can obtain the Cartan-like T-datum $\alpha\left(U_{q}(\hat{\mathfrak{g}}), \ell\right)$, where we replace a normalization of the parameter $u$ appropriately so that $u \in \mathbb{Z}$ and the T-datum satisfies (N1), and we also discard the parameter $\Omega$ in [36, Section 2.4] for twisted $\hat{\mathfrak{g}}$. Explicitly, the T-datum $\alpha\left(U_{q}(\hat{\mathfrak{g}}), \ell\right)$ is given in Table 4, where in the first line we denote by $\alpha\left(X_{n}, \ell\right)$ the T-datum in Theorem 4.12 associated 


\begin{tabular}{l|l} 
type of $\hat{\mathfrak{g}}$ & T-datum $\alpha\left(U_{q}(\hat{\mathfrak{g}}), \ell\right)$ \\
\hline$X_{n}^{(1)}$ & $\alpha\left(X_{n}, \ell\right)$ \\
$A_{2 n-2}^{(2)}$ & $\alpha\left(A_{\ell-1} \otimes C_{n}\right)$ \\
$A_{2 n}^{(2)}$ & $\alpha\left(A_{\ell-1} \otimes T_{n}\right)$ \\
$D_{n+1}^{(2)}$ & $\alpha\left(A_{\ell-1} \otimes B_{n}\right)$ \\
$E_{6}^{(2)}$ & $\alpha\left(A_{\ell-1} \otimes F_{4}\right)$ \\
$D_{4}^{(3)}$ & $\alpha\left(A_{\ell-1} \otimes G_{2}\right)$
\end{tabular}

TABLE 4. T-data associated with quantum affine algebras.

with the Cartan matrix of type $X_{n}$ and the integer $\ell$, and in the remaining lines we denote by $\alpha(Y \otimes Z)$ the T-datum obtained by the tensor product construction in Example 4.7 from the Cartan matrices of types $Y$ and $Z$. The T-datum $\alpha\left(U_{q}(\hat{\mathfrak{g}}), \ell\right)$ is of finite type for any $U_{q}(\hat{\mathfrak{g}})$ and $\ell$ by the periodicity results in $[33,29,27,28]$. The partition $q$-series of $\alpha\left(U_{q}(\hat{\mathfrak{g}}), \ell\right)$ divided by a product of the Dedekind eta coincide with the $q$-series version of the fermionic formulas defined in [24, Section 5]. They conjectured that these $q$-series coincide with string functions of integrable highest modules of $\hat{\mathfrak{g}}$ [24, Conjecutre 5.3]. If this conjecture holds, Conjecture 5.14 for $\alpha\left(U_{q}(\hat{\mathfrak{g}}), \ell\right)$ follows from the results by Kac and Peterson [30].

Example 5.20 ( $q$-series from Nil-DAHA). Let $X_{n}$ be the type of a finite type Cartan matrix, and $p$ be an integer with $p \geq 2$. Consider the T-datum $\alpha\left(X_{n} \otimes A_{p-1}\right)$, where the meaning of this notation is the same as that in Example 5.19. This is of finite type by [33]. Then the partition $q$-series of $\alpha\left(X_{n} \otimes A_{p-1}\right)$ are special cases of the $q$-series studied by Cherednik and Feigin in the theory of Fourier transform of nilpotent double affine Hecke algebras [5, Corollary 1.3]. In fact, they proved that their $q$-series are modular functions [5, Theorem 2.3].

\section{REFERENCES}

1. George E. Andrews, An analytic generalization of the Rogers-Ramanujan identities for odd moduli, Proc. Nat. Acad. Sci. U.S.A. 71 (1974), 4082-4085. MR 351985

2. Arkady Berenstein and Andrei Zelevinsky, Quantum cluster algebras, Adv. Math. 195 (2005), no. 2, 405-455. MR 2146350

3. M. Bershtein, P. Gavrylenko, and A. Marshakov, Cluster integrable systems, q-Painlevé equations and their quantization, J. High Energy Phys. (2018), no. 2, 077, front matter+33. MR 3789593

4. Peigen Cao and Fang $\mathrm{Li}$, The enough g-pairs property and denominator vectors of cluster algebras, arXiv preprint arXiv:1803.05281 (2018).

5. Ivan Cherednik and Boris Feigin, Rogers-Ramanujan type identities and Nil-DAHA, Adv. Math. 248 (2013), $1050-1088$. MR 3107536

6. Philippe Di Francesco and Rinat Kedem, Q-systems as cluster algebras. II. Cartan matrix of finite type and the polynomial property, Lett. Math. Phys. 89 (2009), no. 3, 183-216. MR 2551179

7. _ Q-systems, heaps, paths and cluster positivity, Comm. Math. Phys. 293 (2010), no. 3, 727-802. MR 2566162

8. Miroslav Fiedler and Vlastimil Pták, On matrices with non-positive off-diagonal elements and positive principal minors, Czechoslovak Math. J. 12 (87) (1962), 382-400. MR 0142565

9. Vladimir V. Fock and Alexander B. Goncharov, Cluster ensembles, quantization and the dilogarithm, Ann. Sci. Éc. Norm. Supér. (4) 42 (2009), no. 6, 865-930. MR 2567745

10. Sergey Fomin, Lauren Williams, and Andrei Zelevinsky, Introduction to cluster algebras. chapters 1-3, arXiv preprint arXiv:1608.05735 (2016).

11. Sergey Fomin and Andrei Zelevinsky, Cluster algebras. I. Foundations, J. Amer. Math. Soc. 15 (2002), no. 2, 497-529. MR 1887642

12.

13. __ Y Y-systems and generalized associahedra, Ann. of Math. (2) 158 (2003), no. 3, 977-1018. MR 2031858

14. _ Cluster algebras. IV. Coefficients, Compos. Math. 143 (2007), no. 1, 112-164. MR 2295199

15. Allan P. Fordy and Andrew Hone, Discrete integrable systems and Poisson algebras from cluster maps, Comm. Math. Phys. 325 (2014), no. 2, 527-584. MR 3148096 
16. Allan P. Fordy and Robert J. Marsh, Cluster mutation-periodic quivers and associated Laurent sequences, J. Algebraic Combin. 34 (2011), no. 1, 19-66. MR 2805200

17. Pavel Galashin and Pavlo Pylyavskyy, Quivers with subadditive labelings: classification and integrability, arXiv preprint arXiv:1606.04878 (2016).

18. _ Quivers with additive labelings: classification and algebraic entropy, arXiv preprint arXiv:1704.05024 (2017).

19. - The classification of Zamolodchikov periodic quivers, Amer. J. Math. 141 (2019), no. 2, 447-484. MR 3928042

20. Michael Gekhtman, Michael Shapiro, Serge Tabachnikov, and Alek Vainshtein, Integrable cluster dynamics of directed networks and pentagram maps, Adv. Math. 300 (2016), 390-450. MR 3534837

21. Michael Gekhtman, Michael Shapiro, and Alek Vainshtein, On the properties of the exchange graph of a cluster algebra, Math. Res. Lett. 15 (2008), no. 2, 321-330. MR 2385644

22. Max Glick, The pentagram map and Y-patterns, Adv. Math. 227 (2011), no. 2, 1019-1045. MR 2793031

23. Mark Gross, Paul Hacking, Sean Keel, and Maxim Kontsevich, Canonical bases for cluster algebras, J. Amer. Math. Soc. 31 (2018), no. 2, 497-608. MR 3758151

24. Goro Hatayama, Atsuo Kuniba, Masato Okado, Taichiro Takagi, and Zengo Tsuboi, Paths, crystals and fermionic formulae, MathPhys odyssey, 2001, Prog. Math. Phys., vol. 23, Birkhäuser Boston, Boston, MA, 2002, pp. $205-272$. MR 1903978

25. David Hernandez, Drinfeld coproduct, quantum fusion tensor category and applications, Proc. Lond. Math. Soc. (3) 95 (2007), no. 3, 567-608. MR 2368277

26. Andrew N. W. Hone and Rei Inoue, Discrete Painlevé equations from Y-systems, J. Phys. A 47 (2014), no. 47, 474007, 26. MR 3279998

27. Rei Inoue, Osamu Iyama, Bernhard Keller, Atsuo Kuniba, and Tomoki Nakanishi, Periodicities of T-systems and Ysystems, dilogarithm identities, and cluster algebras I: type $B_{r}$, Publ. Res. Inst. Math. Sci. 49 (2013), no. 1, 1-42. MR 3029994

28. - Periodicities of T-systems and $Y$-systems, dilogarithm identities, and cluster algebras II: types $C_{r}, F_{4}$, and $G_{2}$, Publ. Res. Inst. Math. Sci. 49 (2013), no. 1, 43-85. MR 3029995

29. Rei Inoue, Osamu Iyama, Atsuo Kuniba, Tomoki Nakanishi, and Junji Suzuki, Periodicities of T-systems and $Y$-systems, Nagoya Math. J. 197 (2010), 59-174. MR 2649278

30. Victor G. Kac and Dale H. Peterson, Infinite-dimensional Lie algebras, theta functions and modular forms, Adv. in Math. 53 (1984), no. 2, 125-264. MR 750341

31. Akishi Kato and Yuji Terashima, Quiver mutation loops and partition q-series, Comm. Math. Phys. 336 (2015), no. 2, 811-830. MR 3322387

32. Rinat Kedem, Q-systems as cluster algebras, J. Phys. A 41 (2008), no. 19, 194011, 14. MR 2452184

33. Bernhard Keller, The periodicity conjecture for pairs of Dynkin diagrams, Ann. of Math. (2) 177 (2013), no. 1, 111-170. MR 2999039

34. A. Kuniba and T. Nakanishi, Spectra in conformal field theories from the Rogers dilogarithm, Modern Phys. Lett. A 7 (1992), no. 37, 3487-3494. MR 1192727

35. Atsuo Kuniba, Tomoki Nakanishi, and Junji Suzuki, T-systems and $Y$-systems for quantum affinizations of quantum Kac-Moody algebras, SIGMA Symmetry Integrability Geom. Methods Appl. 5 (2009), Paper 108, 23. MR 2591898

36. T- T-systems and Y-systems in integrable systems, J. Phys. A 44 (2011), no. 10, 103001, 146. MR 2773889

37. Chul-hee Lee, Nahm's conjecture and Y-systems, Commun. Number Theory Phys. 7 (2013), no. 1, 1-14. MR 3108771

38. Kyungyong Lee and Ralf Schiffler, Positivity for cluster algebras, Ann. of Math. (2) 182 (2015), no. 1, 73-125. MR 3374957

39. James Mc Laughlin, Andrew V Sills, and Peter Zimmer, Rogers-ramanujan-slater type identities, the electronic journal of combinatorics 1000 (2008), 15-31.

40. Werner Nahm, Conformal field theory and torsion elements of the Bloch group, Frontiers in number theory, physics, and geometry. II, Springer, Berlin, 2007, pp. 67-132. MR 2290759

41. Tomoki Nakanishi, Periodicities in cluster algebras and dilogarithm identities, Representations of algebras and related topics, EMS Ser. Congr. Rep., Eur. Math. Soc., Zürich, 2011, pp. 407-443. MR 2931902

42. — T-systems, Y-systems, and cluster algebras: tamely laced case, New trends in quantum integrable systems, World Sci. Publ., Hackensack, NJ, 2011, pp. 325-355. MR 2767952

43. _ Synchronicity phenomenon in cluster patterns, arXiv preprint arXiv:1906.12036 (2019).

44. Tomoki Nakanishi and Salvatore Stella, Wonder of sine-Gordon Y-systems, Trans. Amer. Math. Soc. 368 (2016), no. 10, 6835-6886. MR 3471079

45. Naoto Okubo, Bilinear equations and q-discrete Painlevé equations satisfied by variables and coefficients in cluster algebras, J. Phys. A 48 (2015), no. 35, 355201, 25. MR 3400882

46. F. Ravanini, A. Valleriani, and R. Tateo, Dynkin TBAs, Internat. J. Modern Phys. A 8 (1993), no. 10, $1707-1727$. MR 1216231

47. Nathan Reading and Salvatore Stella, Initial-seed recursions and dualities for d-vectors, Pacific J. Math. 293 (2018), no. 1, 179-206. MR 3724242 
48. R. Tateo, New functional dilogarithm identities and sine-Gordon Y-systems, Phys. Lett. B 355 (1995), no. 1-2, 157-164. MR 1343426

49. Masha Vlasenko and Sander Zwegers, Nahm's conjecture: asymptotic computations and counterexamples, Commun. Number Theory Phys. 5 (2011), no. 3, 617-642. MR 2864462

50. Don Zagier, The dilogarithm function, Frontiers in number theory, physics, and geometry. II, Springer, Berlin, 2007, pp. 3-65. MR 2290758

51. Al. B. Zamolodchikov, On the thermodynamic Bethe ansatz equations for reflectionless ADE scattering theories, Phys. Lett. B 253 (1991), no. 3-4, 391-394. MR 1092210

Department of Mathematical and Computing Science, Tokyo Institute of Technology, 2-12-1 Ookayama, MEguro-Ku, TOKYO 152-8550, JAPAN.

E-mail address: mizuno.y.aj@m.titech.ac.jp 